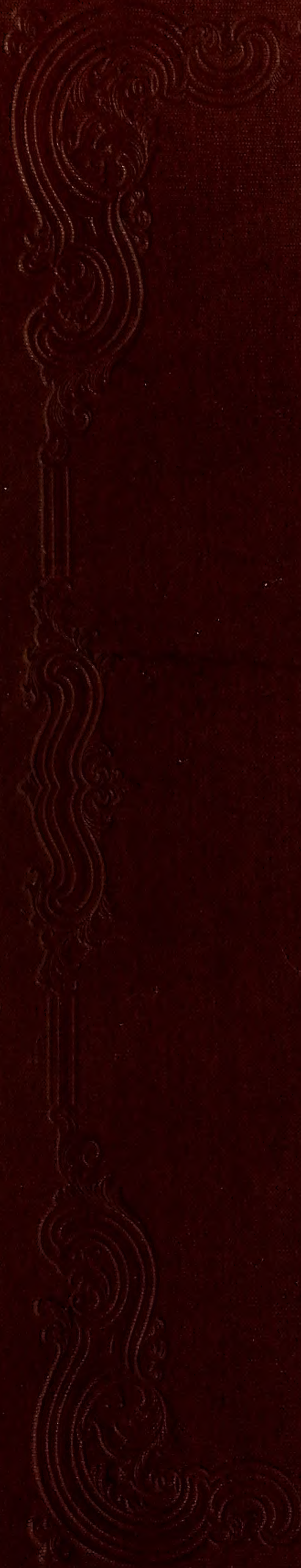
as $\left(\frac{1}{4}\right.$ (3) $\frac{1}{4}$ 
HARVARD UNIVERSITY.

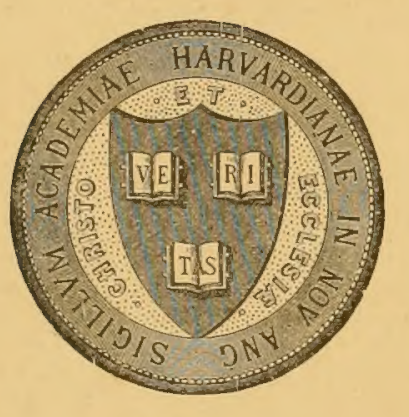

LIBRARY OF THE

MUSEUM OF COMPARATIVE ZOÖLOGY.

1726.

Bought:

OCtober 14,1904. 


SUITE DE L'OUVRAGE:

SYSTĖME SILURIEN DU CENTRE DE LA BOHĖME PAR JOACHIM BARRANDE EDITÉ AUX FRAIS DU FONDS BARRANDE.

\title{
FLORE DÉVONIENNE
}

\section{DE L'ÉTAGE H DE BARRANDE}

\author{
PUBLIÉ PAR
}

\author{
PROF. Dr H. POTONIÉ ET Dr CH. BERNARD. \\ (BERLIN.) \\ (GENÈVE.)
}

AVEC 150 FIGURES DANS LE TEXTE.

EN COMMISSION CHEZ

RAIMUND GERHARD, ANCIENNE MAISON WOLFGANG GERHARD 



\section{Historique.}

Jusqu'ici la flore des terrains siluriens et dévoniens n'a pas encore été étudiée à un point de vue général et un tel travail s'imposait, car les auteurs qui s'occupent de la flore fossile de ces formations éprouvent les plus grandes difficultés à se retrouver au milieu de la littérature confuse et très disséminée qui existe sur ce sujet.

Mais pour pouvoir faire ce travail d'ensemble, une monographie vraiment utile des plantes de ces régions, il fallait, cela va sans dire, que tous les débris connus fussent étudiés sérieusement, que les différentes stations fussent examinées en une étude comparative et critique, et que de cette comparaison ressortît à coup sûr ce qu'il est permis d'affirmer et ce qu'il est possible de supposer.

L'un de nous [Potonié, *) Silur- und Culmflora, pages 3-4] avait émis déjà cette opinion, et il commençait ce travail d'ensemble par l'étude de la flore fossile du Harz et de Magdebourg en ajoutant que la flore du Dévonien moyen de Bohême (Étage $\mathbf{H}-\mathbf{h} \mathbf{1}$ du Silurien de Barrande) devait être étudiée ensuite pour suivre la même direction. M. le Prof. Anton Fritsch lui proposa de publier le résultat de ses recherches sur la Bohême aux frais du fonds Barrande.

Les géologues s'intéressèrent à la région qui nous occupe depuis 1846, date à laquelle J. Barrande publia sa "Note préliminaire sur le Système silurien et les Trilobites de Bohême". Mais il ne s'y préoccupait pas de la question de la flore, et des fossiles végétaux sont pour la première fois signalés dans ce soi-disant Silurien par Barrande en 1852 (Système silurien du centre de la Bohême, Volume I). C'est à la page 81 qu'il donne les „caractères géognostiques de l'étage des schistes "culminans H." Il n'avait, dit-il, pas considéré cet étage dans sa publication de 1846, car il le regardait alors comme le couronnement supérieur de l'étage $\mathbf{G}$. Et il ajoute plus loin que près de Hostím**), sur la rive gauche du ruisseau, il a observé, au milieu des schistes, une petite couche de $3-4^{\mathrm{cm}}$ de houille. "Les bancs schisteux nous montrent ça et là des empreintes, soit de Fucoides „soit de plantes peu distinctes. Ce sont les seuls vestiges du règne végétal que nous ayons ren"contrés dans notre division supérieure."

Dans la description paléontologique de l'étage des quartzites D, il avait dit (page 71): "Toutes „les traces relatives au règne végétal se réduisent à des Fucoides qu'on observe à toute hauteur "à partir de la base de l'étage, mais dont les impressions sont toujours très indistinctes."

Nous verrons plus loin que Goeppert cite à Loděnice un Chondrites. Loděnice appartiendrait d'après Barrande à l'étage $\mathbf{E}$, dans lequel ce dernier ne signale pas trace de fossiles végétaux.

*) Voir à la fin du travail, dans notre Index bibliographique, le titre complet des travaux cités et les indications nécessaires.

**) M. le Prof. J. J. Jahn fait remarquer dans son travail: Sur le Dévonien de la Bohême centrale, 1903, que cette localité, nommée Hostin par tous les auteurs, s'appelle en réalité Hostím. [Voir plus loin notre note *) page 11.] 
C'est en 1859 que parut le travail de Goeppert sur la flore des formations siluriennes, dévoniennes et du Carbonifère inférieur, travail auquel nous faisons allusion. L'auteur divise les étages siluriens en général, en deux formations et il dit (page 570) avoir rencontré dans la formation silurienne inférieure 17 espèces, dans la formation supérieure 3 espèces, „toutes appartenant à la subdivision "des Fucoides."

A propos de la formation silurienne inférieure de la Bohême, il dit (page 556) qu'elle a pour base des couches rouges ou vertes avec des restes de Fucoides qu'il n'a malheureusement pas encore pu étudier, puis vient la zone primordiale avec, d'après Barrande, la soi-disant faune primordiale. Quant aux espèces rencontrées par lui en Bohême „elles sont au nombre de trois seulement dans les „étages qui nous intéressent, et appartiennent à la famille des Floridées."

$1^{0}$ Chondrites fruticulosus, Goepp. (page 450) trouvé par Schary à Loděnice, de même que la variété $\beta$ subarticulatus, Goepp. Il a pu reconnaître encore dans un de ses échantillons des cellules rondes, et ces cellules entouraient une axe transformé en oxyde rouge de fer.

$2^{0}$ Sphcrococcites Scharyanus, Goepp. (page 454) trouvé également à Loděnice dans l'étage D de Barrande.

$3^{0}$ Enfin dans la formation silurienne supérieure, dans l'étage $\mathbf{E}$ de Barrande, à Dlouhá Hora au sud-est de Beraun il a trouvé Callithamnion Reussianum, Goepp.

Ettingshausen en 1865, page 17, fait du Sphcrococcites Scharyanus, Goepp. un Equisetites Gopperti, Ett.

En 1854 déjả, puis en 1865 et 1866 Barrande avait donné des comptes-rendus plus ou moins longs de ses travaux sur le Silurien de la Bohême, mais il ne s'y occupait que de la faune ou de la disposition stratigraphique du terrain et ne signalait rien de spécial quant à la flore.

Cependant en 1865 parut le vol. III de sa "Défense des Colonies." Les deux premiers volumes avaient, à leur publication, soulevé de violentes critiques sur la conception que se faisait Barrande de ses "Colonies de Bohême". Ces critiques sont réfutées dans le vol. III; le vol. IV, paru en 1870, donne de nouveaux détails et constate que Krejči et Lipold, les principaux adversaires de la théorie de Barrande se sont rangés à sa manière de comprendre les choses. Dans le vol. III, Barrande s'occupe des environs de Hlubočepy où l'on peut reconnaitre le même étage $\mathbf{H}-\mathbf{h} \mathbf{1}$ de Hostím et Srbsko, stations qui nous intéressent plus spécialement. Il donne tout d'abord la division des étages $\mathbf{G}$ et $\mathbf{H}$ dont il veut s'occuper et les divise chacun en trois formations principales dont il indique la diagnose. Nous ne nous arrêterons qu'à la couche $\mathbf{H}$-h $\mathbf{1}$, c'est à dire à la formation qui se trouve à la base de l'étage $\mathbf{H}$, soit en contact avec l'étage $\mathbf{G}$.

Après avoir donné la caractéristique géologique et paléontologique (faune) de ces étages, il dit à propos de la flore (page 52): „Les seules traces distinctes du règne végétal que nous ayons dé„couvertes dans nos étages $\mathbf{G}$ et $\mathbf{H}$, sont des impressions de Fucoides, observées uniquement dans "les couches schisteuses ou siliceuses, et jamais jusqu'ici dans les bancs calcaires. Les plus re"marquables de ces impressions appartiennent à une seule espèce, Fucoides hostinensis". Puis il cite les vestiges végetaux qu'il a rencontrés:

$1^{0}$ Fucoides hostinensis, Barr. trouvé dans l'étage $\mathbf{H}-\mathbf{h} \mathbf{1}$, à Hlubočepy, à Hostím et sous Choteč.

$2^{0}$ Apparence d'écorce charbonnée rencontrée dans l'étage $\mathbf{H}-\mathbf{h} \mathbf{1}$, à Hostím et à Hlubočepy.

$3^{0}$ Couche très mince d'anthracite, à Hostím dans l'étage $\mathbf{H}-\mathbf{h} \mathbf{1}$.

Et plus loin (page 304): „Les seules traces de nature végétale, que nous connaissons dans nos "étages $\mathbf{G}-\mathbf{H}$, sont des Fucoides, principalement représentés par Fucoides hostinensis, Barr. Cette "espèce, très caractérisée par ses rameaux dichotomes et par les apparences d'une grande ténuité ${ }_{n}$ dans sou écorce nous semble avoir joui en Bohême d'une existence extrêmement prolongée, quoique „intermittente". Il la cite dans les étages $\mathbf{D}, \mathbf{E}$ et $\mathbf{G}$ et continue: „Nous la retrouvons plus haut, 
"avec une nouvelle fréquence dans les schistes de la bande h 1, à Hostín, Hlubočep, Choteč et autres "localités. Enfin elle disparaît pour toujours an-dessus de la bande h 1."

„Nous ne connaissons pas de Fucoides dévoniens qui présentent les mêmes apparences que "Fucoides hostinensis. Ainsi les restes de nature végétale qu'offrent nos étages supérieurs $\mathbf{G}-\mathbf{H}$, „tendent à les relier avec nos étages infériekrs, sans établir aucune connexion avec la flore du "terrain dévonien".

Celui-ci, en Thuringe par exemple, a fourni au Dr. Richter*) une flore très remarquable.

Jusqu'ici donc les auteurs n'ont voulu voir dans cette flore du centre de la Bohême que des plantes marines.

Krejči, le premier, en 1879 puis en 1881, décrit des empreintes de plantes terrestres. Il dit que cet étage $\mathbf{H}$ considéré par Kayser et d'autres comme appartenant au Dévonien inférieur a été caractérisé par Barrande comme Silurien et il cite les plantes terrestres vasculaires suivantes, récoltées par Schary et Dusl à Hostím et à Srbsko:

Protopteridium hostinense, $\mathrm{Kr}$.

Protolepidodendron Scharyanum, Kr.

Protolepidodendron Dustianum, Kr.

Equisetites siluricus, Kr.

Puis des restes d'animaux, puis des Fucoides et entre autres Fucoides hostinensis, Barr., très fréquent, graminoïde, à empreintes ramifiées en dichotomies. Enfin à Srbsko, il a trouvé aussi des impressions foliiformes rappelant Cordaites borassifolius, mais qui pourraient être parentes à des Delesserites.

„D'autres Fucoides sont des formes graminoïdes à nervures parallèles, avec une forte nervure "médiane et rappellent les Haliserites du Dévonien. D'autres rappellent les Confervites, d'autres im"pressions enfin sont analogues aux Cystoseira du Tertiaire."

En 1881, dans une nouvelle collection, il retrouve les formes décrites en 1879: Protolepidodendron Dustianum, Kr., dont un exemplaire rappelle un jeune rameau de Lepidodendron Veltheimi. Quelques morceaux montrent de plus grosses cicatrices de feuilles, un peu comme les Knorria. Un morceau de fronde fertile de Fougère se rattache au Protopteridium hostinense, Kr. Puis parmi les Fucoides, il nomme Haliserites zonarioides, Kr., forme qui ressemble au Zonarites digitatus de Nannsfeld. A un autre qui rappelle distinctement la forme du Dévonien de Coblenz nommée Drepanophyllum spinaforme, Goepp., il donne le nom de Haliserites spinosus, Kr. Quelques formes très fréquentes, délicatement ramifiées, sont appelées Chondrites verticillatus, $\mathrm{Kr}$.

Puis en 1882 paraît le travail de Stur, qui nous intéresse plus directement, puisqu'il s'occupe des stations de Srbsko et de Hostím, lesquelles font justement l'objet de nos recherches. Stur est revenu à l'idée que ces plantes de "terrains siluriens" étant les plus anciennes plantes connues doivent être les plus inférieures, et il les considère toutes comme des Algues marines. C'est ainsi que de Protolepidodendron Scharyanum, Kr. et d'Equisetites siluricus, Kr., il fait une Siphonée, Chavvinia Scharyana, Kr. sp. Il l'a rencontrée dans l'étage H-h 1 à Hostím et à Hlubočepy. De Haliserites spinosus, Kr. ex parte, il fait une Laminariée, Lessonia bohemica, Stur, rencontrée dans l'étage $\mathbf{H}-\mathbf{h} \mathbf{1}$ à Srbsko et à Hostím. Le Chondrites verticillatus, Kr. devient le Sporochnus Krejčí, Stur, une Sporochnö̈dée, trouvée à Srbsko dans l'étage H-h 1. Dans le même endroit, il a rencontré une Fucacée, Fucus Nováki; Stur, qui n'est autre chose que le Haliserites spinosus de Krejěí, ex parte.

Il décrit aussi Hostinella hostinensis, Barr. manuscr. comme une Floridée (ordre des Gigartinées) qui se rencontre à Hostím, Hlubočepy et Srbsko, dans l'étage $\mathbf{H}-\mathbf{h} \mathbf{1}$, et qui serait un groupement nouveau des anciennes espèces: Fucoides hostinensis, Barr., Haliserites zonarioides, Kr., ex parte et Protopteridium hostinense, Kr.

*) On sait maintenant que cette flore $d u$ Dr. Richter se trouve dans des schistes appartenant au Culm. 
Dans l'ordre des "Characee pracursores", Stur fait le genre Barrandeina, et plus spécialement B. Dusliana, Kr. spec., qui serait le Protolepidodendron Duslianum, Kr., et qui se rencontre dans l'étage H-h 1 à Srbsko. Des planches illustrent ce travail, ce qui n'est malheureusement pas le cas pour les publications précédentes.

Crié en 1883 ne s'occupe pas directement des plantes dévoniennes de la Bohême, mais il signale un point qu'on avait déjà relevé avant lui, et qui fut depuis confirmé par les observations d'autres auteurs; il dit: "L'existence dès le Silurien supérieur des Cryptogames vasculaires et surtout des "Gymnospermes peut nous surprendre, tant il est vrai qu'un penchant général nous porte à regarder "la simplicité comme le caractère d'une haute ancienneté. Trop souvent en effet, on se figure que la "simplicité qui, relativement à nos procédés analytiques, est antérieure à la complexité, l'est aussi "dans l'ordre des temps; c'est là une erreur dont le naturaliste doit se garder". Il nous a paru bon de consigner ici cette opinion, car c'est en se basant sur l'idée que combat Crié, que les auteurs ont voulu voir souvent des Algues dans ces plantes des terrains primitifs et ont refusé de les considérer comme végétaux supérieurs.

Jusqu'ici la plupart des auteurs avaient considéré ces terrains du centre de lá Bohême comme appartenant au Silurien, et ils s'appuyaient pour cela sur l'opinion de Barrande qui avait force de loi. Mais depuis longtemps la question de la présence du Dévonien dans le centre de la Bohême avait été discutée. Reuss en 1854 avait admis l'hypothèse comme probable que "les couches du Silurien "supérieur du centre de la Bohême pourraient appartenir an Dévonien“. Mais c'est Kayser surtout qui, en 1878, puis en 1884 les caractérisa comme terrains dévoniens. Depuis lors la plupart des auteurs ont adopté cette manière de voir. Les stations les plus discutées, et qui sont précisément celles qui nous intéressent, furent considérées par Neumayr en 1887 comme Dévonien inférieur; actuellement on les considère comme la région supérieure du Dévonien moyen.

En 1890 Schenk, dans le chapitre "Algen" de la "Paléophytologie" du "Zittel's Handluch", après avoir signalé les difficultés que l'on rencontre dans la détermination des Algues fossiles et les erreurs commises dans ce domaine, dit, à la page 234, que beaucoup d'Algues de la série des Caulerpites, dont il cite les noms: la plus grande partie des Palcophycées, Oldhamia, les Chondritées (parmi lesquelles les Algues du Flysch), les Fucoïdées, Spongiophycées, Dictyophycées, la plupart des Algues décrites par Heer dans la „Flora fossilis Helvetiæ“, puis Eophyton, enfin les restes très mal conservés décrits par Stur comme Algues fossiles, sont tous bien plus semblables à des plantes supérieures qu'aux genres d'Algues auxquels ils ont été rattachés. D'autres doivent être considérés comme des traces animales.

En 1892 paraît le livre de Katzer suỉ la géologie de la Bohême. L'auteur remet clairement les choses au point quant à la stratigraphie et à la géologie du pays. Il fait rentrer les stations de Hostím et de Srbsko dans la couche Df du Dévonien moyen. Quant à la flore, qu'il n'a pas étudiée, il s'en rapporte aux travaux précédents et aux espèces décrites par Stur.

En 1894 Solms-Laubach, à propos de plantes dévoniennes d'autres régions, s'arrête en passant aux formations de la Bohême. Diseutant les plantes trouvées là, il cite les noms créés par Stur, mais il se hâte d'ajouter que Stur a établi souvent un parallélisme incompréhensible entre quelquesunes de ses formes et les Algues, par exemple à propos de Barrandeina Dusliana qu'il place dans les Characées, mais que Krejči, avant lui, avait bien plus justement considéré comme Protolcpido. dendron Duslianum. Quant aux autres, Hostinella hostinensis, (Kr.) Stur, considéré par Krejči comme Protopteridium, c'est bien en effet un reste de Fougère. De même Chauvinia Scharyana devrait être bien plutôt prise pour une plante terrestre. Quant à Sporochnus Krejči il ne saurait affirmer s'il doit être pris pour une Algue ou pour une Fougère. Lessonia bohemica et Fucus Nováki enfin, très semblables l'un à l'autre, n'ont pas le moindre rapport avec le genre Lessonia.

En 1896 Rothpletz décrit des „Flysch-Fucö̈den", et parmi d'autres il cite Hostinella hostinensis de Stur, qu'il a pu étudier, dont il donne un dessin et qu’il a examiné au point de vue microscopique. 
Il affirme qu'il a pu y reconnaître des cellules isodiamétriques, et cela lui suffit pour affirmer que ce ne peut être une plante supérieure, mais une Algue. "Quant à dire, comme Stur l'a fait, que c'est "une Floridée, rien ne m'autorise, dit-il, à aller si loin."

Mařik a décrit en 1900 un fragment bien peu net et bien peu complet de Fougère dont il donne une figure et qu'il nomme Sphenopteris Velenovskíi. Ce petit morceau a été trouvé à Hlubočepy dans la couche $\mathbf{h} \mathbf{1}$, et l'auteur' le considère comme une plante terrestre, en partie en se basant sur les précédents travaux de Krejči et de Schenk (qui ont affirmé que les restes trouvés dans ces couches étaient terrestres et non marins et cela avec d'autant plus de raison que les couches en question ont été considérées non plus comme Silurien, mais comme Dévonien), en partie en se basant sur des raisons pétrographiques qui l'ont conduit à concevoir l'étage $\mathbf{H}-\mathbf{h} \mathbf{1}$ comme étant formé de sédiments d'eal douce.

Bayer, qui a donné de ce travail un résumé dans le "Just's Jahresbericht", ajoute à ce sujet que, se basant sur des raisons géologiques, il s'est convaincu que les Algues marines de Stur ne peuvent être autre chose que des Cryptogames vasculaires, et que le fragment de Sphenopteris Velenovskýi ne serait qu'un morceau de feuille de Hostinella hostinensis. Or, il est certain que cette dernière plante appartient aux Ptéridophytes, et très vraiscmblablement aux Fougères. Il ne peut $y$ avoir lì-dessus aucun doute, d'autant moins que Bayer, étudiant des parties carbonisées de cette même plante, fut assez heureux, dit-il, pour mettre en préparation des éléments cellulaires, qui étaient encore très semblables, quoique très désorganisés, à des trachéides de Fougères, par exemple de Pteridium. M. Ie Dr. Bayer promettait de faire, à l'occasion, une petite notice à ce sujet; il n'en a pas eu le loisir, mais dans une très aimable lettre qu'il nous écrivait au commencement de 1903 , il nous confirmait ses précédentes opinions, ainsi que ses observations antérieures. Il a eu l'obligeance de nous faire parvenir les dessins qu'il a faits de ces éléments considérés par lui comme scalariformes. Mais nous ne les reproduisons pas, car nous n'oserions affirmer que ce fussent vraiment des vaisseaux très caractérisés.

Schubert, en 1900, décrit un reste d'Algue du Silurien supérieur de Bohême. Ce fragment très incomplet et assez peu distinct fut considéré par lui comme une Algue, et il se basait, pour affirmer cela, sur les diagnoses macro- et microscopiques. Cette Algue avait été trouvée dans la couche $\mathbf{E}$, à Podol-Dvorce, couche plus ancienne que la couche $\mathbf{H}$, preuve qu'il y avait des débris végétaux plus anciens que les nôtres; clle fut nommée Chondrites Moldavae.

Au milieu de cette divergence d'opinions, il était nécessaire de remettre les choses au point, et de voir ce qu'il était possible de supposer ou d'affirmer en l'état actuel de nos connaissances. Qu'il y ait des fossiles végétaux dans l'étage dévonien moyen et notamment dans l'étage $\mathbf{H}$ de Barrande, cela n'a rien d'étonnant, puisque nous avons vu que Schubert en a trouvé dans l'étage $\mathbf{E}$, qui est plus ancien, et puisque l'étage silurien supérieur du Harz possède des restes de Lycopodiales. Mais il résulte de nos recherches, comme on le verra plus loin, que l'on peut affirmer que tous les restes en question appartiennent à des plantes terrestres. De plus, il est probable que le nombre des espèces est peu nombreux. Tout le reste ne peut être qu'un ensemble de suppositions qui découleront des faits que nous avons pu observer et que nous discuterons dans le cours du travail.

Quant à la disposition des coizches de l'étage $\mathbf{H}$ de Bohême, nous empruntons à la publication de M. le Prof. J. J. Jahn les renseignements suivants:

"Barrande a divisé son étage $\mathbf{H}$ en 3 bandes:

"L’inférieure h1 (d'après Krejči = „Schiefer von Srbsko") se compose principalement d'argiles „de diverses apparences. Cette bande, d'après Krejči, a une épaisseur de 20 à 60 mètres; c'est la "partie inférieure de cette bande qui contient des restes abondants de plantes et d'animaux." 


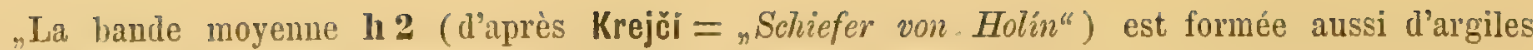
galternant avec des schistes quartzeux. Krejči estime l'épaisseur de cette bande à peu près "it 150-200 mètres. Il ne s'y trouve aucun fossile."

"La bande supérieure $\mathbf{h} \mathbf{3}$ (d'après Krejči $=$ „Schiefer von Hostin") se compose seulement d'ar"giles et ne comporte pas de fossiles. La puissance de cette bande est, d'après Krejči, d'environ "20-40 mètres."

"A la surface, ces schistes de $\mathbf{H}$ s'étendent sur un espace restreint, à peu près entre Prague „et Beraun. Aujourd'hui, on n'accepte pas cette classification de Barrande en 3 bandes, car, $1^{0}$ il "n'y a de fossiles qu'à la base de l'étage II, et $2^{0}$ les prétendues différences pétrographiques ne "suffisent pas pour en faire des horizons particuliers. Les principales stations où l'on trouve des "fossiles sont, d'après la littérature: Srbsko, Hostím et Hlubočepy. Les autres (Třebotov, Holín, „Choteč, Boubová, Karlstein) n'ont fourni que des restes assez rares et relativement peu importants.*) "D'après les animaux fossiles que II. le Prof. E. Holzapfel étudie, il ne reste aucun doute que la „bande H-h 1 de Barrande, c'est-à-dire la seule partie où l'on rencontre des fossiles, n'appartienne "ì la région supérieure du Dévonien moyen."

Dans cette même publication de M. Jahn se trouve un résumé de M. Holzapfel sur la faune fossile de la région, et aussi quelques lignes se rapportant à notre travail, entre autres, la liste des espèces végétales dont la discussion va suivre.

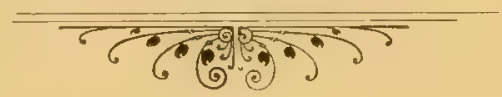

*) Monsieur Jahn vient de m'envoyer de beaux restes de plantes fossiles qu'il a trouvés récemment à Boubová, mais qui ne démontrent rien de plus que ceux de Srbsko etc. $P$. 


\section{Introduction.}

Pour l'exécution du présent travail, nous avons utilisé des matériaux qui nous ont été aimablement prêtés par les musées ou les collectionneurs suivants; nous profitons de l'occasion pour leur exprimer tous nos remerciements.

1. Museum des Königreiches Bölmen. Prag (Prague). (Section géologique et paléontologique, Prof. Dr. Anton Fritsch.)

2. Geologisches Institut der deutschen Universität. Prag. (Dir. Prof. Dr. G. C. Laube.)

3. Geologisches Institut der böhmischon Universität. Prag. (Dir. Prof. Dr. J. N. Woldřich.)

4. Deutsche technische Hochschule. Prag. (Dir. Prof. Dr. Fr. Wähner.)

5. Böhmische technische Hochschule. Prag. (Dir. Prof. Dr. A. Slavík.)

6. Hofmuseum. Wien (Vienne). (Dir. Prof. Th. Fuchs.)

7. Wiener Reichsanstalt. (Dir. Hofrat Dr. G. Stache.)

8. Universität Wien. (Dir. Prof. Dr. L. Suess.)

9. Monsieur M. Dusl, à Beraun. (Collection qui renferme un grand nombre d'originaux de Stur).

10. Monsieur Wenzel Tomášelo, fabricant à Karlstein, Böhmen. (Sa collection a été donnée ensuite à la "böhmische technische Hochschule de Brïm".)

Enfin, le service géologique de Berlin a fait pratiquer des fouilles à Srbsko, près Beraun, et a acheté le grand nombre de fossiles trouvés là. Ces fouilles ont été pratiquées par M. Vincent Marek, sous la direction de M. le Prof. J. J. Jahn, à Brünn.

Nous avons eu entre les mains les dessins de M. le Prof. Vlček, de Wittingau, qui avait entrepris l'étude de cette flore et l'avait laissée inachevée.

Les fossiles des collections sus-nommées se rapportent directement au Dévonien de la Bohême, et plus spécialement à l'étage $\mathbf{I}-\mathbf{h} \mathbf{1}$, de Hostím, Srbsko, etc. Ces matériaux nous ont été envoyés à la suite d'un voyage que Potonié, en vue de ce travail et sous les auspices du service géologique de Prusse, a entrepris à Vienne, à Prague, à Beraun et à Dresde, afin de voir les collections, et de comparer les matériaux.

Nous devons remercier aussi M. W. Gothan, qui travaille au laboratoire de Potonié, et qui a bien voulu faire les macérations de houille et les préparations microscopiques. Il a effectué, en outre, la plupart des dessins. MM. E. Ohmann, C. Krohse et G. Hoffmann, à Berlin, ont fait les autres.

Nous voyons que jusqu'ici les avis ont été très partagés, tant sur la manière de concevoir la géologie pure de ce terrain, l'âge des couches et leurs rapports entre elles, que quant à la paléontologie et à la détermination des espèces végétales. Et cette divergence d'opinions est très compréhensible, si l'on songe que nous sommes ici en présence d'un terrain dont l'allochtonie est très nettement caractérisée. Divers auteurs, Mařik et Bayer entre autres, avaient affirmé que cette flore de l'étage 
H--l 1 était composée de débris clarriés. Nous avons pu relever plusieurs des caractères que Potonié a énoncés comme devant déterminer l'allochtonie d'un terrain. Entre autres, la mauvaise conservation des empreintes végétales, l'absence presque totale de folioles de Fougères, et, avant tout, la présence, dans plusieurs de nos échantillons, des formations décrites sous le nom de "Häcksel". En allemand, on appelle $H a ̈ c k s e l$, dans le langage courant, cette paille hachée en fins débris et qu'on mélange à l'avoine des chevaux. Par analogie, on a nommé de même ces accumulations de débris finement broyés, comme hachés par le transport de l'eau, presque tous à peu près de la même dimension dans un schiste donné, et qui finissent par s'accumuler dans les sédiments qui caractérisent les formations allochtones. Nous n'avons pas d'expression française à appliquer à cette formation, et nous continuerons à la nomwer du mot allemand très explicite. Ces débris, qui sont plus fins à mesure quils sont charriés à une distance plus considérable, peuvent être reınarquablement orientés, tous dans la même direction, s’ils ont été déposés en un endroit où le courant de l'eau était encore relativement fort. Le Häcksel, du reste, est décrit dans le "Lehrbuch" de Potonié, et dans sa "Silur- und Kulmflora des Harzes"; nous y renvoyons le lecteur, de même que pour l'exposé plus complet et la discussion des caractères qui déterminent l'allochtonie d'un terrain.

Nous avons rencontré, dans les differentes stations qui nous intéressent, beaucoup de ces Häcksel; de très bons échantillons, trouvés à Hostím et déterminés dans les collections comme Hostinella hostinensis, montraient des empreintes végétales broyées en très fins morceaux, ou en fragments plus gros, mais toujours toutes de la même grandeur dans un même échantillon. Un autre caractère des terrains allochtones est la présence d'animaux marins mélangés ì des plantes terrestres. Nous avons pu relever ce détail dans les objets que nous avons étudiés (fig. 7, 55, 63, 83, 93, 112).

Puisque nous sommes, daus l'étage $\mathbf{H}$ - h1, en présence d'un terrain allochtone bien caractérisé, il ne faudra pas se baser sur l'absence de limbes de Fougères pour affirmer, a priori, que les débris conservés ne peuvent appartenir à ce groupe de plantes; bien au contraire, les tissus mous des folioles ayant été broyés, pourris, ont disparu, ne laissant que les parties dures, les pétioles, les liges, etc, que les auteurs ont considérés comme des Algues. C'est dans des conditions, qui ne se rencontrent malheureusement qu'avec trop de rareté, que nous trouvons parfois dans les terrains allochtones des restes très reconnaissables de feuilles de Fougères, qui viennent alors élucider nos doutes. C'est ainsi que dans quelques-uns de nos échantillons nous avons pu constater de jeunes feuilles encore enroulées en crosses et qui ont vraisemblablement appartenu à des Fougères. Mais ces débris complets, pour les raisons exposées ci-dessus, ne sont jamais en aussi grande ahondance dans ces terrains que dans les schistes autochtones paléozoïques. Les auteurs, du moins plusieurs par'mi les premiers qui se sont occupés de la question, ont voulu voir dans nos fossiles des Algues, et, comme nous l'avons dit, ils basaient leur opinion sur le fait qu'ils pensaient ces terrains siluriens et dévoniens devoir être considérés comme les plus anciens et devoir posséder les végétaux les plus anciens, donc les plus simples, donc des Algues. Ils oubliaient que les terrains où l'on rencontrait exclusivement ces végétaux anciens et simples sont trop vieux pour avoir pu nous conserver des empreintes végétales. Nous avons déjà relevé les erreurs qui peuvent découler d'une telle manière de voir, et nous avons cité à ce sujet l'opinion de Criẻ et d'autres (voir aussi le Lehrbucl de Potonié, pp. 106, 107, 353,362).

Qu'il y ait eu des Algues marines autochtones dans ces formations qui ne nous ont transmis que des débris allochtones de plantes terrestres, cela ne fait pas l'ombre d'un doute, et cela est confirmé par la présence au milieu de nos restes végétaux terrestres, et sur les mêmes pièces, de fossiles d'animaux marins (Orthoceras, Trilobites, etc., fig. 7, 55, 63, 83, 93, 112). Mais les tissus plus délicats des Algues marines n’ont pas rencontré les conditions nécessaires pour se fixer et arriver jusqu'à nous, et il est bien certain que les restes que nous avons eus sous les yeux pour la plupart du moins - n'appartiennent pas à ces Thallophytes, mais bien à des Ptéridophytes, 
qui ont été amenés d'assez loin, ont été broyés en morceaux plus ou moins gros, et rendus presque méconnaissables.

Nous ne connaissons pas de flore dévonienne ou silurienne bien certainement autochtone, et il est très évident que des doutes subsisteront sur les fossiles de ces terrains jusqu'à ce que des recherches ultérieures aient amené la découverte d'une flore dévonienne autochtone aussi bien définie que la plupart de celles du Carboniférien, et à laquelle on pourra rapporter les débris allochtones comnus. Ises indications claires manquent, qui permettent de caractériser bien nettement ces débris, de les réunir entre eux et de les rapporter à des genres et à des espèces connus. C'est pour cette raison que nous n'avons pas voulu baptiser nos types de noms précis qui n'auraient été que du schéma et de l'arbitraire. Le système analytique utilisé en paléobotanique pour les flores autochtones, par exemple pour celles du Carboniférien, ne pouvant être appliqué ici par suite du manque d'empreintes convenables, à plus forte raison nous ne pouvons pas appliquer le système analytique utilisé en botanique, et qui nous eût permis de rattacher nos restes fossiles à telles ou telles espèces encore existantes, puisqu'on ne peut même pas le faire avec certitude pour beaucoup de débris houillers autochtones. Nous en avons été réduits à faire des suppositions sur les formes que nous avions sous les yeux, et quand nous rencontrions des fragments de houille, nous les faisions macérer par le procédé connu (oxydation par le chlorate de potasse et l'acide nitrique) pour en faire l'analyse microscopique qui pouvait nous fournir d'utiles indications. Ces suppositions nous ont conduits aux résultats probables suivants que nous aurons à discuter plus loin.

Ces débris appartiennent avec assez de vraisemblance à des Fougères, à des Protocalamariacées, ì des Lycopodiales, à des Psilotacées, à des Bothrodendracées, à des Ginkgoacées, et à des Conifères. En outre, il y avait des fragments encore indéterminables, mais assez nets et assez curieux pour mériter d'être signalés.

Il est à supposer que les espèces vraies ne seront pas aussi nombreuses que celles qui ont été décrites jusqu'ici, ni que celles que nous dénommons; car il est peu probable qu'un grand nombre de plantes aient été emportées par les eaux pour être disposées en couches allochtones. En effet, l'eau qui a eu l'occasion d'arracher des plantes les aura enlevées toujours aux mêmes endroits, comme nous le constatons aujourd'hui; ou bien elle aura attaqué des associations de plantes vivant dans des conditions analogues, donc peu variées.

Souvent les noms que nous utiliserons, pour les raisons que nous venons de dire, et parce que nous ne voulons rien préciser, seront ceux utilisés par les anciens paléobotanistes; ils n'auront pour but que de nous faciliter l'exposé de nos recherches et la description de nos échantillons, et d'indiquer seulement les analogies que nous avons pu constater. Nous répétons que nous avons fait plus de noms sans doute qu'il n'y a de vraies espèces; mais ceci dans l'intention surtout de définir' les échantillons dont nous parlons et que nous ne savons comment rattacher entre eux.

Par exemple, pour le genre Spiropteris, que nous décrivons plus bas, on comprendra que l'attribution de cette plante à un genre déterminé serait sans valeur, car il s'agit de jeunes frondes, et il est impossible jusqu'ici d'affirmer que ces frondes aient appartenu à tel genre ou à tel autre. Et ce doute subsistera jusqu'à ce qu'on ait pu découvrir les pinnules qui détermineront cette Fougère par exemple comme un Archcopteris ou un Rhodea, genres qui caractérisent les terrains dévoniens et siluriens.

Tous ces fossiles sont généralement colorés en jaune-brunâtre et se distinguent nettement sur le fond grisâtre assez foncé de la pierre. Ce sont des infiltrations minérales (de fer?) qui les colorent de cette manière. Mais il est un point sur lequel nous devons attirer l'attention des observateurs, c'est que les fossiles, trouvés en grande quantité dans ces stations, ont fait l'objet d'un commerce actif, et que les marchands, pour donner plus de valeur à leurs échantillons, les ont peints avec de la sépia, en suivant quelquefois les traces de l'empreinte, ou bien en se laissant guider souvent par 
leur imagination. Comme une telle pratique peut être cause d'erreurs, et qu'elle l'a été du reste, il sera bon de toujours laver soigneusement, arant de les examiner, les pièces des collections, pièces qui, comme nous arous pu le constater dans un grand nombre de musées, ont subi la petite opération que nous renons de signaler.

Nous lécrirons donc les espèces provisoires suivantes:

\section{Fougères :}

1. Spiropteris hostimensis, $\mathrm{P}$. et $\mathrm{B}$.

2. Rhodea (?) hostimensis, P. et B.

3. Hostimella hostimensis, a) typica, P. et B.

B) rhodereformis, $\mathrm{P}$. et $\mathrm{B}$.

\section{Equisėtales:}

4. Cf. Asterocalamites scrobiculatus, (Schloth.) Zeiller.

\section{Lycopodiales.}

\section{Psilotacées (?):}

5. Pseudosporochmus Krejči, (Stur ex parte) P. et B.

Bothrodendracées (?):

6. Protolepidodendron Tiartsteini, P. et B.

7 Protolepidodendron Scharyanum, Kr.

8. Ulodendion (?) hostimense, P. et IB.

\section{Lycopodiacées :}

9. Lycopodites hostimensis, P. et B.

Gymnospermes (?).

Ginkgoacées (?):

10. Barrandeina Dusliana, (Kr.) Stur.

\section{Conifères (?):}

11. Psitophyton spinosum, (Kr. ex parte) P. et B.

12. Psilophyton bohemicum, (Kr. ex parte) $\mathrm{P}$. et B.

13. Coniferites Fritschi, P. et B.

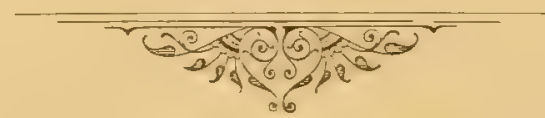




\title{
Deseription des espèces.
}

\author{
Spiropteris hostimensis, *) P. et B.
}

Fig. 1 à 6 .

\author{
Fucoides hostinensis, Barr. ex parte.**) \\ Protopteridium hostincnse, (Barr.) Kr. \\ Hostinella hostinensis, Barr. (in Stur) ex parte. \\ ? Barrandites, Ettingshausen (in Stur).***)
}

Nous avons rencontré dans plusieurs de uos échantillons des empreintes très remarquables, que nous considérons comme des restes de Fougères. Stur, en baptisant ces fossiles d'après Barrande Hostinella hostinensis, en avait fait des Algues, et notamment des Gigartinere, de l'ordre des Floridées. Il est vrai qu'on peut rencontrer dans les Algues rouges des enroulements rappelant ceux des frondes jeunes de Fougères, mais les rappelant très vaguement, tandis qu'ici nous sommes en présence de crosses trop bien caractérisées pour qu'il puisse y avoir des doutes. Les fossiles que nous avons examinés provenaient de Srbsko, de Hostím, etc. (étage $\mathbf{H}-\mathbf{h} \mathbf{1}$ de Barrande), et les mêmes caractères se répétaient, typiques pour les diverses stations (fig. 1 à 6). Stur, p. $332-333$, nie que ces restes soient terrestres; il dit qu'Ettingshausen lui a écrit, en 1881, pour l'informer qu'il avait trouvé dans les plantes de Barrande une Fougère, proche parente de Schizaea (c'est le Hostinella hostinensis, Barr., in Stur), et pour laquelle il propose le nom de Barrandites. Ce type, ainsi que le Lepidodendron Veltheimianum (?)

*) Comme nous l'arons dit plus haut [voir la note**) p. 11] la station de Hostín des auteurs s'appelle en réalit Hostím (voir Jahn). Les espèces baptisées d'après cette station s'appelleront donc hostimensis. De plus, le genre Hostinella des auteurs, genre que nous étudierons plus loin, sera appelé, selon la proposition de M. Jahn, Hostimella. Nous sommes heureux de pouvoir faire ce changement, afin d'établir plus clairement, au milieu de la synonymie embrouillée, les types que nous décrivons. On ne confondra done pas notre Hostimella hostimensis, par exemple, arec les nombreuses formes qui ont été appelées jusqu'ici Hustinella hostinensis et qui comprenaient non seulement potre Hostimella hostimensis, mais encore des types rangés par nous sous d'autres noms. D'après les règles de la nomenclature en vigueur maintenant chez les botanistes, nous aurions dû accepter, pour l'exemple que nous citons, l'ancien nom, en l'accompagnant de la mention: Barr. ex parte. Mais ces règles sont établies en botanique sur des données tout à fait différentes de celles fournies par la paléobotanique, oì l'on doit créer des espèces le plus sourent sur des débris ou sur des états différents de conservation; les règles de la nomenclature botanique n'ayant pas été établies en vue et sous l'influence des besoins des paléobotanistes, ceux-ci doivent s'y soumettre le plus possible, mais seront sourent obligés d'agir d'après leur appréciation personvelle.

**) Il est très difficile d'établir. la synonymie de ces types, les noms les plus variés ayant été appliqués aux formes les plus différentes; souvent des types ont été séparés, quoique spécifiquement très identiques, sous deux noms différents, tandis que deux restes très dissemblables étaient réunis dans une même espèce. D'autre part, ce qui augmente les difficultés est le fait que, les dessins manquant dans la plupart des travaux, il est généralement très difficile de concevoir, d'après les descriptions des auteurs, quelles formes ils ont définies par un nom donné; souvent, en outre, les auteurs citent un nom sans préciser à quoi ce nom se rapporte.

***) Nous mettons un point d'interrogation devant cette détermination, car Stur, qui l'indique, nous dit que Etlingshausen l'a appliquée à un échantillon de Hostinella qu'il avait eu entre les mains, mais il ne nous dit pas ¿̀ laquelle des nombreuses formes de Hostinella ce nom se rapportait. 
qu'Ettingshausen signalait aussi, permettait à cet auteur de conclure dans sa lettre qu'il se trouvait en présence d'une "très vieille flore terrestre".

Il nous semble que Krejči avait été beaucoup mieux inspiré que Stur, en baptisant ces débris dn nom de Protopteridium hostinense.

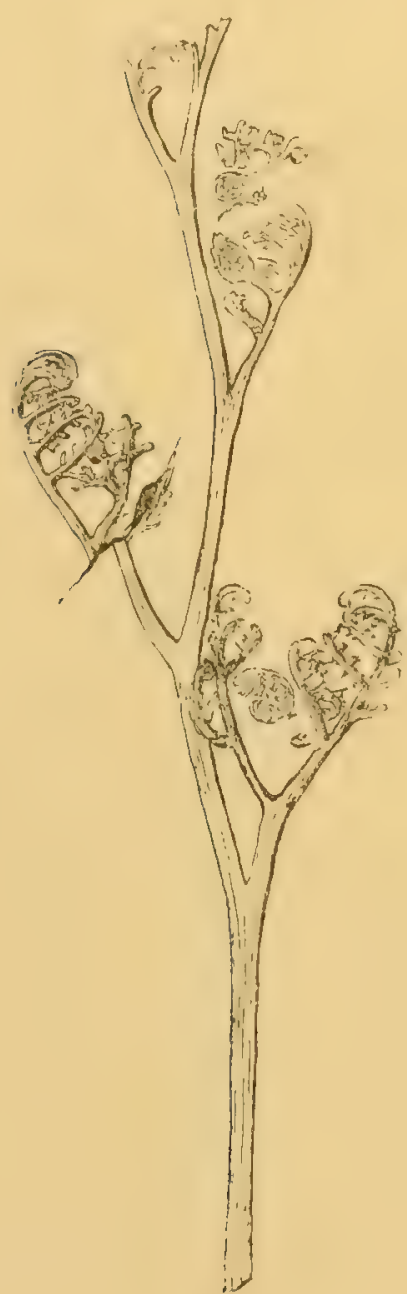

Fig. 1.

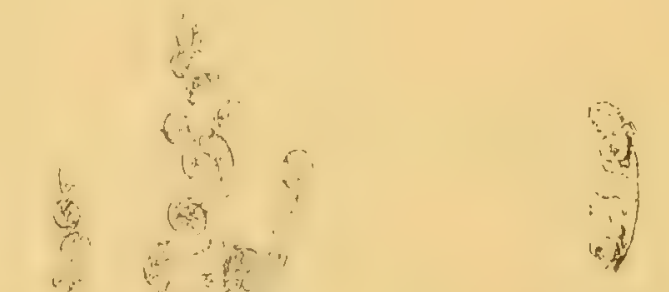

Fir. :.

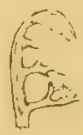

Fig. 6.

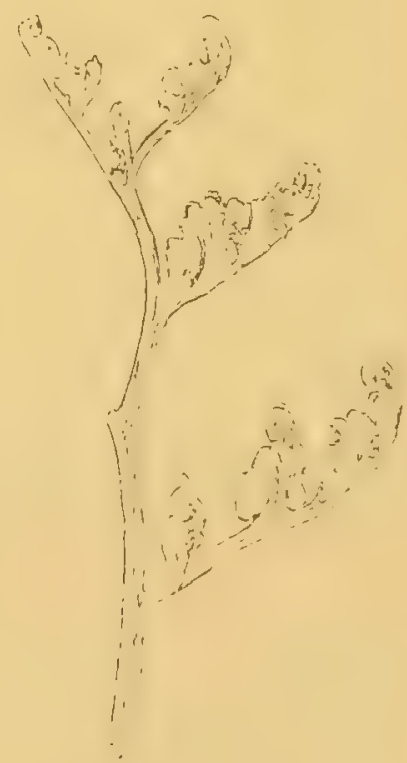

Fig. 4.

Fig. 1-6. Spiropteris hostimensis, P. et B. Grandeur naturelle.

1. Srbsko. (Deutsche Univ. Prag. La contre-empreinte chez M. DusI à Beraun.) Original de Stur: Pl. II, fig. 7. Axe dichotomisé. Enroulement des pinnules des différents ordres.

$\because 2$. Srbsko. (Böhmische Univ. Prag.) Infiltrations enlevant aux détails toute leur netteté.

4. Hostim. (Böhmische Univ. Prag.) Original de Stur, Pl. II, fig. 8. Enroulement des pinnules. Infiltrations.

5. Ilostim. (Univ. Wien.) Enroulement des pinnules.

6. Srbsko. (Geol. Landes-Museum, Berlin.) Enroulement des pinnules.

Cependant nous ne voulons pas préciser autant et attribuer à cette espèce le nom d'un type bien connu; aussi nous contenterons-nous d'adopter l'ancien nom qui servait aux paléontologistes pour désigner les frondes jeunes de Fougères encore enroulées, et qu'ils ne savaient à quel genre attribuer, soit Spiropteris, espèce S. hostimensis. 
Souvent des infiltrations minérales (fer?) empêchent de distinguer avec clarté la forme des pinnules et des crosses (fig. 2-5). Stur admettait ces échantillons enroulés en crosses comme les rameaux fertiles d'Algues dont il décrivait d'autre part les tronćs et les rameaux stériles; et il s'ippuyait, pour affirmer cela, sur l'analogie que présentent ses échantillons avec des Gigartina et plus spécialement avec G. pistillata, dont il donne une figure. Mais tandis que, dans cette Floridée, l'enroulement des rameaux est à peine appréciable, chez les échantillons de Stur comme dans les nôtres, il est très net pour les pinnules.

D'autre part, Stur a rattaché à Hostinella hostinensis des fragments qu'il considérait comme les troncs de cette Algue. Nous n'avons pas très bien saisi, ni dans les descriptions, ni dans les dessins de Slur, ni dans les fossiles originaux qu'a décrits cet auteur et que nous avons eus entre les mains, les raisons qui lui ont fait séparer ces soi-disant trones de Floridées du genre qu'il nomme Sporochnus Krejčíi, et que nous aurons à discuter plus loin.

\section{Hhoden (?) hostimensis, P. et $B$.}

Fig. 7-10.

Fucoides hostinensis, Barr. ex parte.

Haliserites zonarioides, Kr. ex parte.

Hostinella hostinensis, Barr. (in Stur) ex parte.

Nous ne savons pour le moment à quel genre rapporter exactement le Spiropteris dont nous venons de parler. Mais nous avons pu relever d'autres renseignements qui pourraient jeter quelque

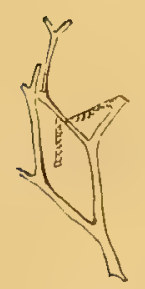

Fig. 7.

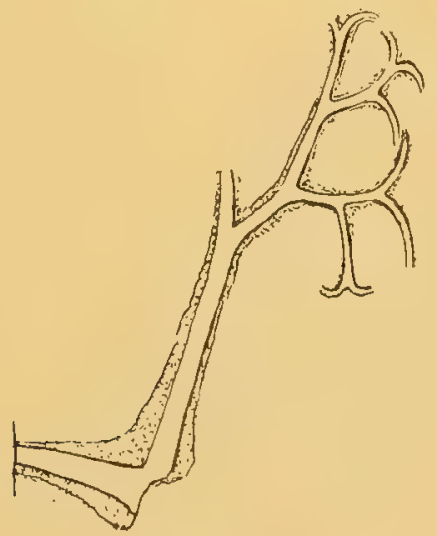

Fig. 8.

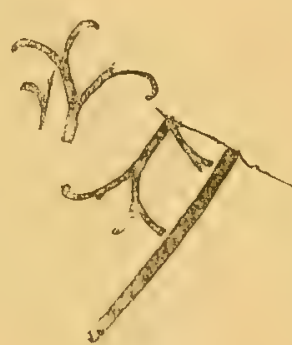

Fig. 9.

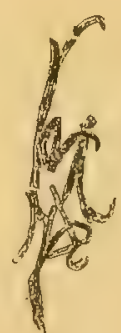

Fig. 10.

Fig. 7-10. Rhodea (?) hostimensis, P, et B. Grandeur naturelle.

7. Dlouhá Hora. (K. K. Geol. Reichsanstalt.) Axe dichotomique. Graptolithes.

8. Hostim. (Univ. Wien.) Axes dichotomisés et enroulés, entourés d'infiltrations minérales jaunâtres (fer?) établissant comme un double contour.

9. Hostîm. (Deutsche Univ, Prag.) Axes dichotomisés et enroulés.

10. Srbsko. (Collection de Y. Dusl à Beraun.) Original de Stur, Pl. IV, fig. $6 a$. Axes dichotomisés et enroulés.

lumière sur la question: Dans d'autres pièces (fig. 7-10) troavées anssi à Hostím, à Srbslio, etc., on peut rencontrer les formes à rameaux dichotomiques et à extrémités nettement courbées (fig. 8-10), qui caractérisent le genre Rhodea, auquel nous rattachons pour le moment nos échantillons sous le nom de Rhodea (?) hostimensis. Stur considérait ces restes comme les dernières ramifications des rameaux stériles de son Hostinella, tandis que ceux que nous décrivons plus haut 
comme Spiropteris étaient pour lui les rameaux fertiles de ce même Hostinella. Si nous arrivions à trouver des formes présentant à la fois ces apparences de Rhodea et les frondes décrites ci-dessus, il va sans dire que les deux types devraient être réunis sous un même nom, ce que nous n'avons pas osé faire dans l'état actuel de nos connaissances.

Souvent les empreintes de ces stations sont entourées d'infiltrations jaunâtres (de fer probablement) (fig. 8). Dans une de nos pièces, trouvée à Dlouhá Hora dans l'étage $\mathbf{H}-\mathbf{h} \mathbf{2}$ de Barrande, nous avons pu voir le rameau de Fougère, accompagné de Graptolithes, preuve de l'allochtonie du terrain (fig. 7).

Dawson considère, en 1862, comme "restes de pinnules fertiles" d'un Cyclopteris incerta (!) des empreintes qui rappellent d'assez près les extrémités un peu enroulées de notre Rhodea (?) hostimensis.

Ces caractères de la flore de Srbsko et de Hostím sont importants pour définir ces terrains comme appartenant au Dévonien. Jusqu'ici les auteurs ne sétaient appuyés pour l'affirmer que sur la stratigraphie de la région et sur la faune. Nous apportons un argument de plus, tiré de l'examen de la flore. En effet, nous voyons nos fossiles être très voisins, sinon identiques ì ceux caractérisant les autres formations lévoniennes. C'est ainsi que notre Rhodea (?) hostimensis est très semblable (identique?) au $R$. condrusorum (Sphenopteris condrusorum, Gilk., Rhacophyton condrusonum, Crép.) qui a été signalé comme caractéristique des „Lenneschiefer" du Dévonien. Il est assez semblable aussi an Splenopteridium Keilhaui, que Nathorst a décrit pour la flore fossile de l'île des Ours, et qui rappelle par l'écartement de ses pinnules Rhodea condrusorum. Il est voisin de la Fougère que Solms désigne comme caractéristique des "Lenneschiefer der Gegend ron Gräfrath am Niederrhein." et qui n'est autre que Rhoden condrusomm. Solms n'admet pas l'identité de son type avec le nôtre; mais il relève leur analogie et signale aussi le rapport qui existe entre Hostimella hostimensis et les Rhodea, quand il dit, p. 88: „De même, nous ne pourrions établir un parallélisme „aussi immédiat entre notre reste et le Sphenopteris condrusorum, Gilk. et le Hostinella hostinensis, "Stur, qui lui est très semblable."

Ilostimells hostimensis, *) $P$. et $B$.

Fig. $11-51$.

Fucoides hostinensis, Barr. ex parte.**)

IIaliserites zonarioides, Kr. ex parte.

Hostinella hostinensis, Barr. (in Stur) ex parte.

? Sphenopteridium Keilhaui, Nath.

? Barrandites, Ettingsh. (in Stur) ex parte.*;

Nous plaçons ici des restes de deux apparences et qui paraissent très caractéristiques pour les terrains dévoniens, car outre que nous les trouvons très fréquents dans l'étage $\mathbf{H}-\mathbf{h} \mathbf{1}$, de Barrande, à Srbsko aussi bien qu'à Hostím, nous en voyons d'assez semblables cités d'une part par Solms-Laubach (Lenneschiefer), d'autre part par Nathorst (Ile des Ours).

1. a) typica. P. et B. Fig. $11-46$.

Il s'agit d'axes (tiges? rachis?) quelquefois assez larges (jusqu'à $10^{\mathrm{mm}}$ et plus), se ramifiant de façon très régulièrement dichotomique; souvent une des deux ramifications prend l'avantage sur l'autre, s'allonge, se place dans la direction de la branclie mère, et rejette la branche soeur de côté (en allemand: Uebergipfelung); les dichotomies suivantes seront soumises au même phénomène, mais

\footnotetext{
*) Voir notre note *), p. 11.

**) Voir notre note **), p. 11.

***) Toir notre note ***), p. 11
} 

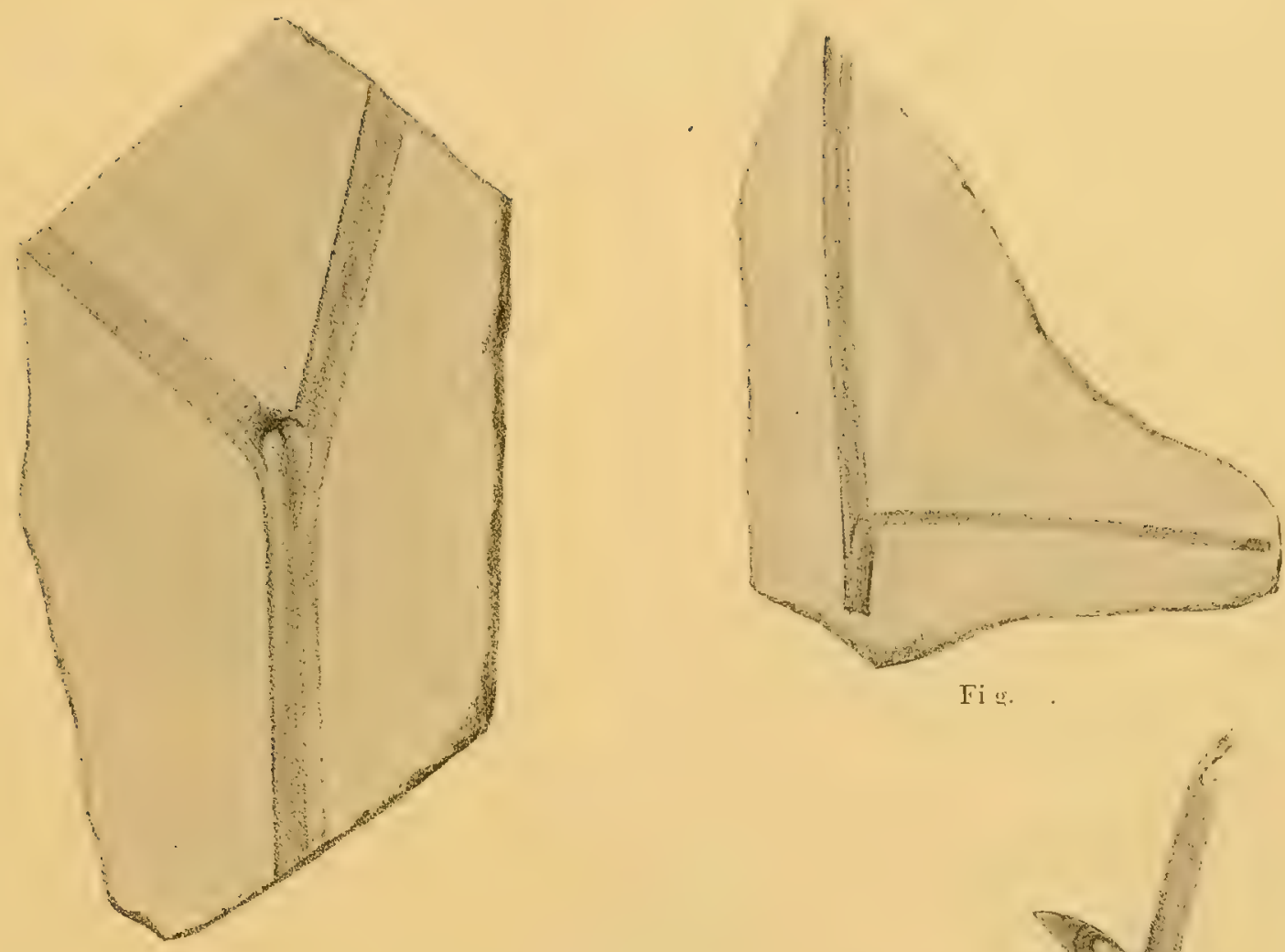

Fi

Fig. 11.

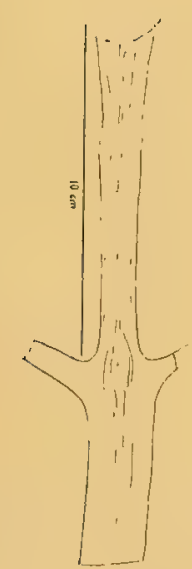

Fig. 14 .

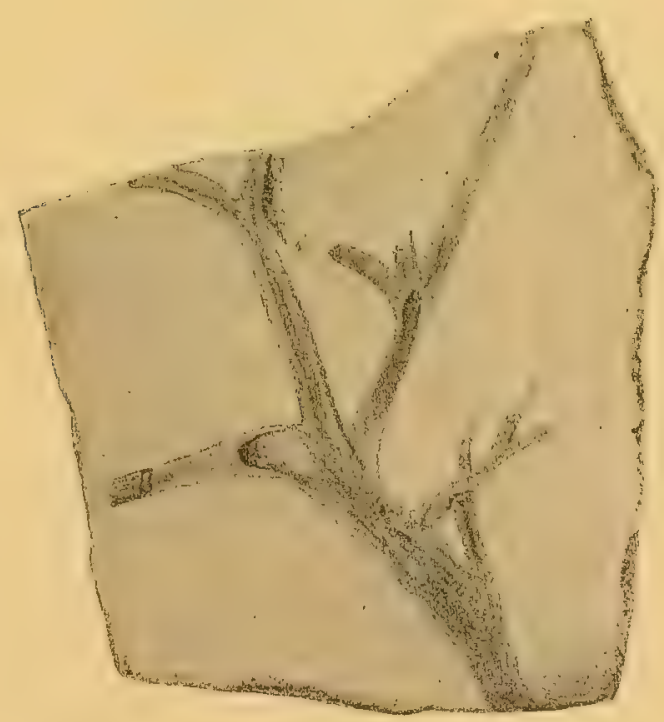

Fig. 13.

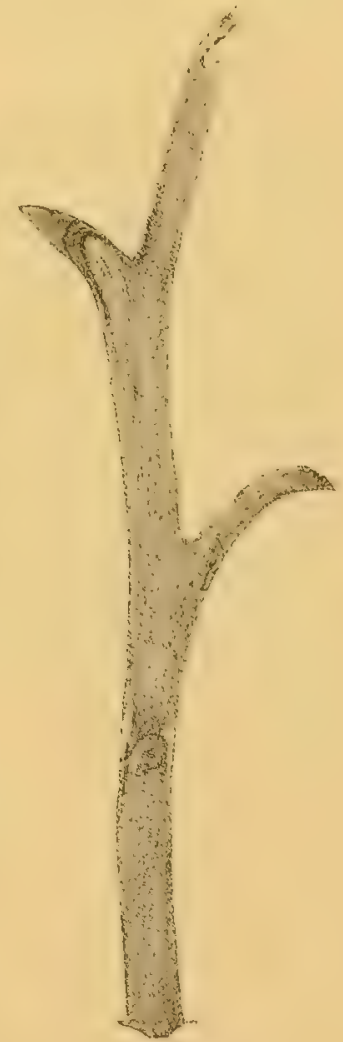

Fig. 15.

Fig. 11-15. Hostimella Lostimensis, P. et B. $\propto$ ) typica

11. Hostim. (Geol. Landesmuseum, Berlin.) Axe dichotomique; deux „bourgeons“ $\frac{1}{1}$.

12. Sibsko. (K. k. Reichsanstalt, Wien.) Axe, "bourgeons" $\frac{1}{1}$.

13. Srbsko. (K. k. Reichsanstalt, Wien.) Axe, "bourgeon" $\frac{1}{l}$.

14. Hostim. (K. k. Reichsanstalt, Wien.) Axe, "bourgeon"? $\frac{1}{3}$.

15. Srbsko. (K. k. Reichsanstalt, Wien.) Axe, "bourgeon", "Uebergipfelung". Points superficiels $\frac{1}{1}$ 
généralement du côté opposé, et l'ou aura un axe plus ou moins droit, avec des ramifications en apparence latérales, alterni-pennées (fig. 12, 15,21, 25 et 33, surtout 36 et 37). Cet objet serait presque une démonstration palpable de la théorie de Potoniẻ, (voir Pericaulom-Theorie) qui fait dériver toutes les ramifications pennées (dans le sens le plus large du mot: grappes, etc.) de dichotomies plus ou moins rapides.
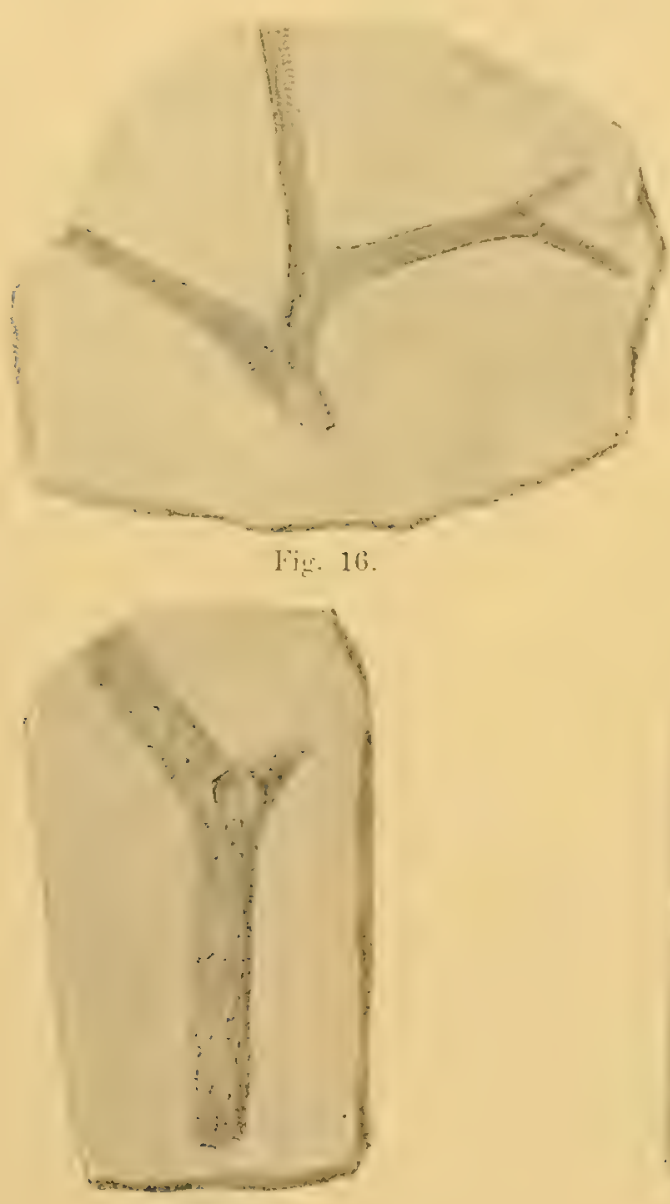

Fiv. 18

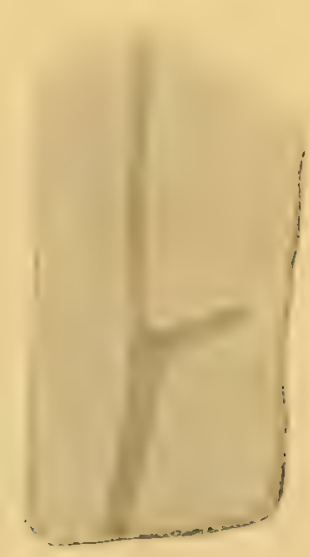

Fig: 19.

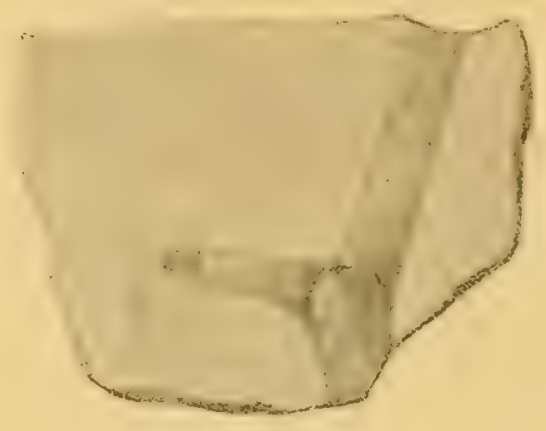

liig. 17.

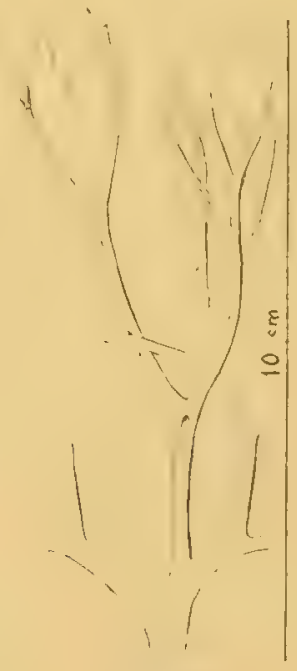

Fi…21).

Fig. 16-20. Ilostimella hostimensis, P. et B. a) typica.

16. Srbsko. (II. k. Reichsanstalt, Wien). Axe, ,bourgeon". Stries transversales sur la surface $\frac{1}{1}$.

17. Srbsko. (, n $n$ T.

18. Hostim. ( $"$ " $"$ Points superficiels $\frac{1}{1}$.

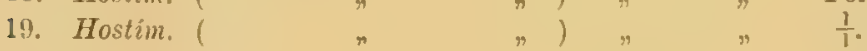

20. Srbstio. ( $"$ ") Axe, dichotomie, "bourgeons ${ }^{k} \frac{2}{3}$.

Nous avons pu voir souvent sur ces formes des axes centraux très nets, qui permettent de repousser absolument l'idée d'une A]gue (fig. 13, 23, 27, 28, 29, 31, 33, 36, 46); dans d'autres cis, sur la surface de ces empreintes, nous remarquons de petites stries transversales courtes et nombreuses (fig. 16), ou bien des stries longitudinales plus ou moins longues (fig. 14, 34, 35), ou plus souvent (fig. 15, 18), de petits points assez rapprochés que nous ne sarons guère ì quoi homologuer; mais ce qui caractérise le mieux ces échantillons (fig. 11-23, 28, 32, 33, 35-37, 41): c'est la présence, sur les axes de divers ordres, d'empreintes particulières rappelabt un peu la trace 


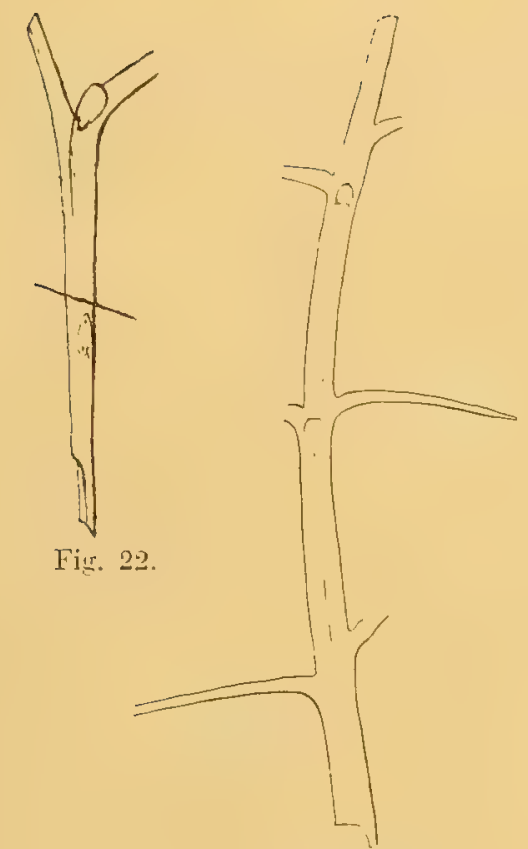

Fig. 21.

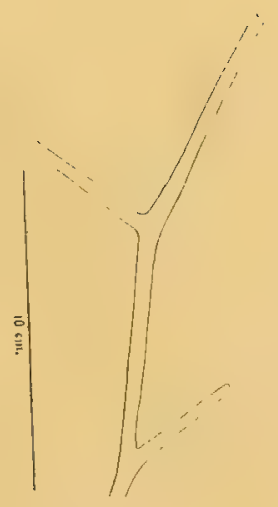

Fig. 27.

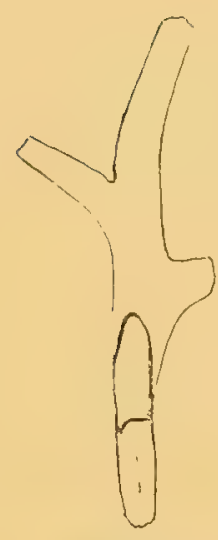

Fig. 24.

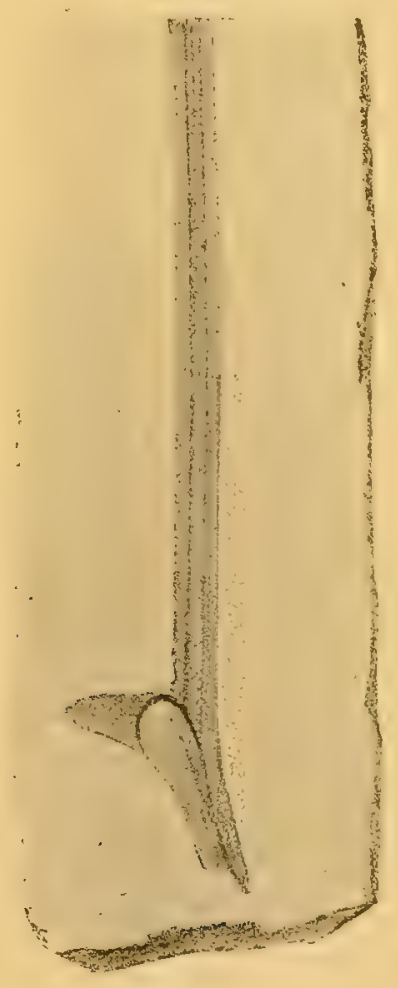

Fig. 28.

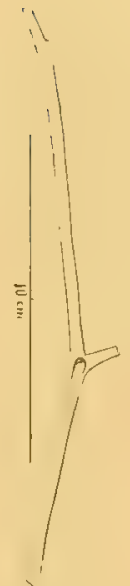

Fig. 23.

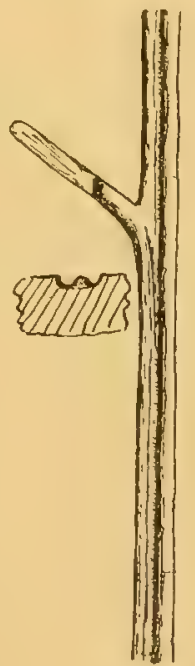

Fig. 29.

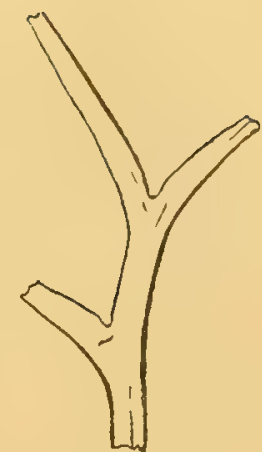

Fig. 25 .

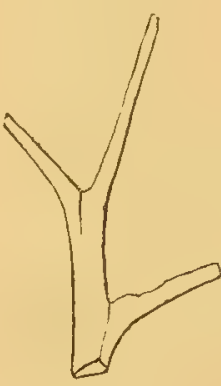

Fig. 26.

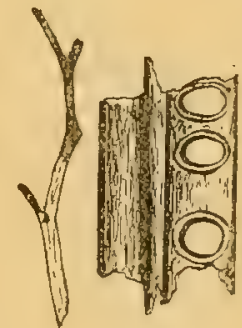

Fig. 30.

Fig. 21-30. Hostimella hostimensis, P. et B. «) typica.

21. Srbsko. (K. k. deutsche Univ. Prag.) Original de Stur, Pl. IV, fig. 1. Axe ramifié par „Uebergipfelung" , „bourgeons" $\frac{1}{1}$.

22. Hostim. (Univ. Wien.) Dichotomie, "bourgeon" $\frac{1}{1}$.

23. Hostim. (Reichsanstalt, Wien.) Axe central, „bourgeons“ $\frac{1}{3}$

24. Srbsko. (Böhm. Univ. Prag.) $\frac{1}{1}$.

25. Srbsko. (Reichsanstalt, Wien.) $\frac{1}{1}$.

26. Karlstein. (Reichsanstalt, Wien.) $\frac{1}{1}$.

27. Srbsko. (K. k. geol. Reichsanstalt.) $\frac{1}{3}$.

28. Srbsko. ( $" \#)$ "Bourgeons", axe central $\frac{1}{2}$.

29. (Univ. Wien.) Axe central très net $\frac{1}{1}$. Nous avons représenté à gauche une section du fossile avec axe central encadré de deux rainures.

30. Fragment avec couche de houille $\frac{1}{1}$; à droite les éléments du bois obtenus par macération; ponctuations aréolées à large contour interne, et disposées en une série; fibres $\frac{25}{1} \frac{0}{6}$. 
que pourrait laisser un bourgeon. Ces soi-disant „bourgeons", qui ont souvent une apparence kuorrioïde, nous ont paru être plutôt le résultat de dichotomies très rapides, mais dont une seule branche se serait développée, et dont l'autre aurait avorté. Nous voyons une preuve de ceci dans le fait que ces „bourgeons", qui sont le plus généralement à la base des deux rameaux de la dichotomie, exactement à l'angle, peuvent se trouver aussi sur les rameaux eux-mêmes, et ceci à des hauteurs plus ou moins grandes (fig. 13, 15, 22, 36, 38).

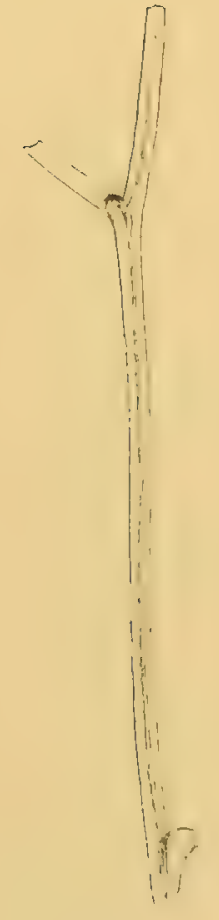

Fig. 33.

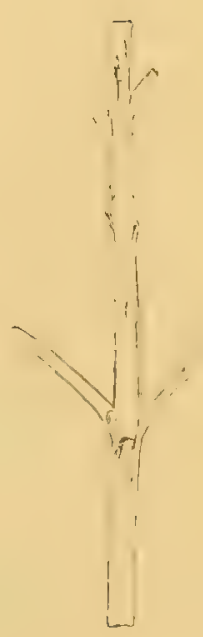

Fig. 32.

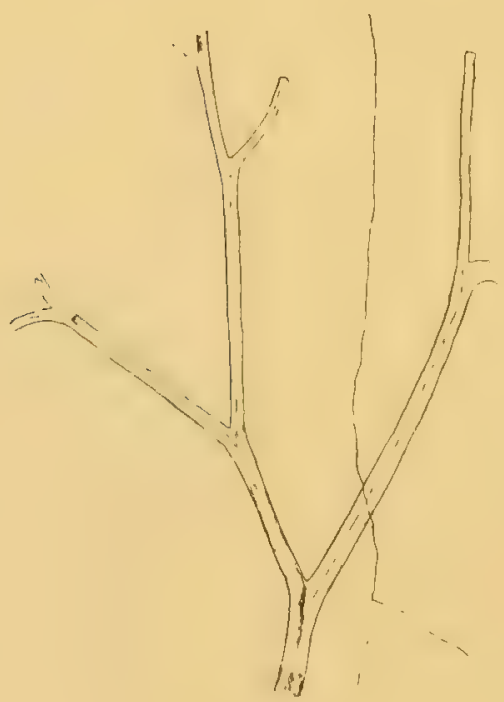

Fị. 31 .

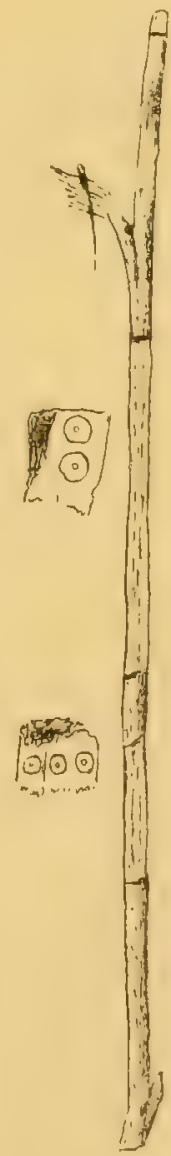

Fig. 34 .

Fig. 31-34. IIostimella hostimensis, P. et B. $\alpha$ ) typica.

31. Srbsko. (K. k. Naturh. Hofmuseum, Wien.) Axe central, dichotomies $\frac{1}{2}$.

32. Srbsko. (Geol, Landesmuseum, Berlin.) Axe ramifié $\frac{1}{2}$.

33. Srbsko. (", ) "Bourgeons". Axe central $\frac{1}{2}$.

34. Srbskio. (Univ. Wien.) Fragment avec houille $\frac{1}{1}$. A gauche éléments aréolés obtenus par macération $2 \frac{5}{1} 0$.

De plus, nous avons pu, par les procédés de macération connus, obtenir des cellules très bien caractérisées par des ponctuations aréolées, ¿̀ contour interne petit (fig. 34) ou grand (fig. 30), et par des fibres (fig. 30). Nous ne voulons pas affirmer que les ponctuations à large contour interne ne soient pas des états différents de conservation de ponctuations à aréoles plus petites. Ces stéréohydroïdes sont une preuve de plus qu'il s'agit ici de plantes terrestres et non d'Algues marines.

Les ramifications peuvent parfois se diriger de façon curieuse, irrégulièrement dans des sens différents (fig. 37); peut-être n'est-ce qu'accidentel. 


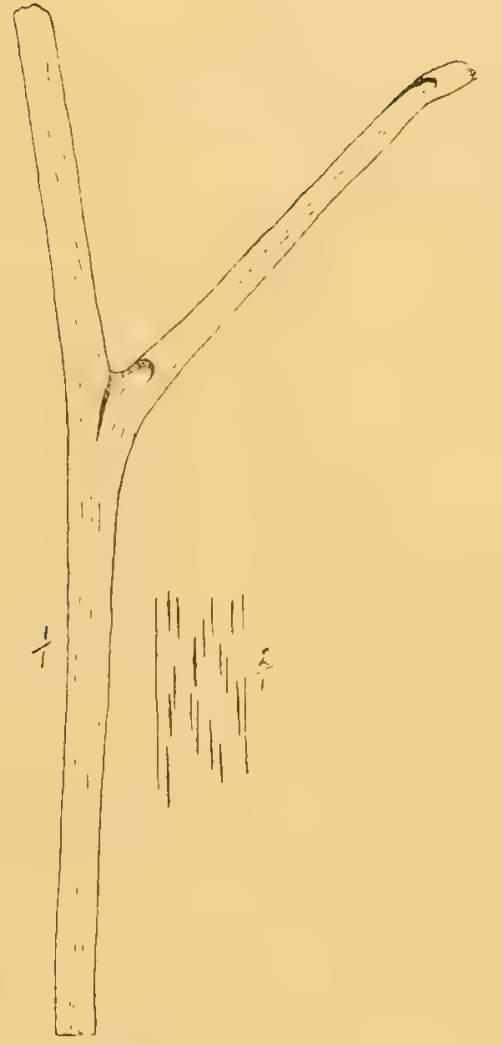

Fig. 35

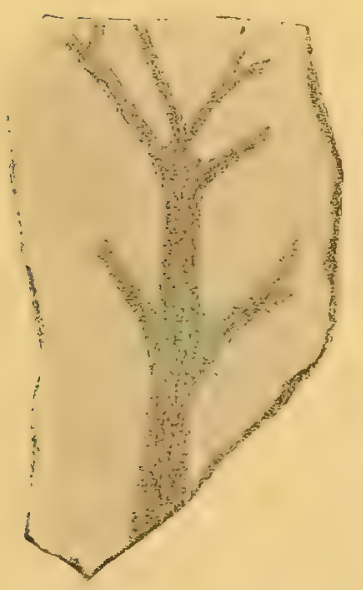

Fig. 39.

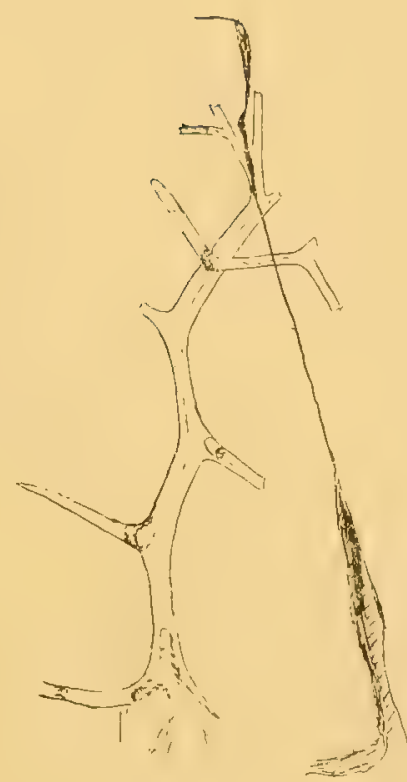

Fig. 37.

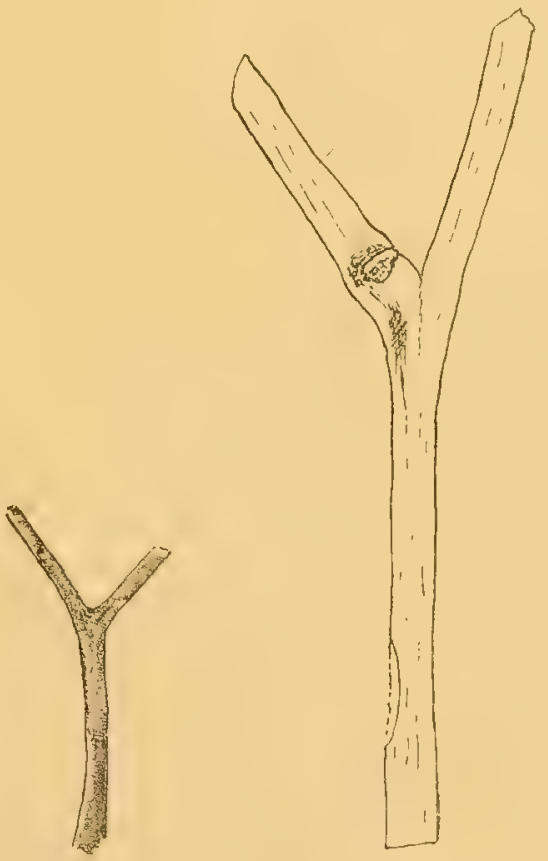

Fig. 40 .

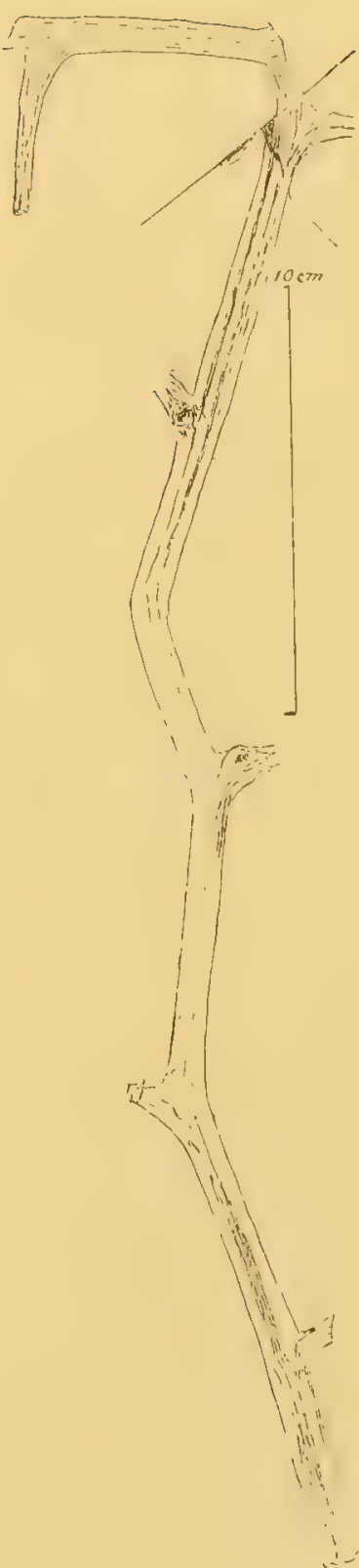

Fig. 36.

Fig. 35-40. Hostimella hostimensis, P. et B. $\alpha$ ) typica.

35. Hostim. (Univ. Wien.) Axe dichotome, „bourgeon" $\frac{1}{1}$; à droite portion avec stries longitudinales $\frac{2}{1}$.

36. Srbsko. (Geol. Landesmuseum, Berlin.) „Uebergipfelung". Env. $\frac{i}{2}$

37. Srbsko. (Hofmuseum, Wien.) "Uebergipfelung". Les ramifications ne sont pas toutes dirigées dans le même sens. "Bourgeons" $\frac{1}{2}$.

38. Hostim. (Univ. Wien.) „Bourgeon" placé assez haut sur la branche de gauche $\frac{1}{1}$

39. Srbsko. (Geol. Landesmuseum, Berlin.) Ramifications nombreuses $\frac{1}{1}$.

40. Srbsko. Dichotomie, „bourgeons ${ }^{\star 1} \frac{1}{1}$. 
La fig. 11 montre deux „bourgeons" côte à côte, chacun à la lase d'une des ramifications, et accolés l'un à l'autre; on pourrait aussi penser à un bourgeon dichotome.

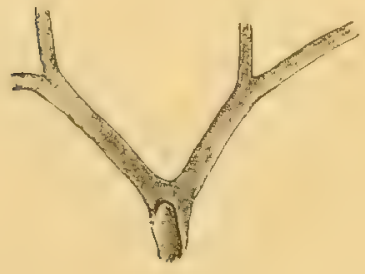

Fig. 41 .

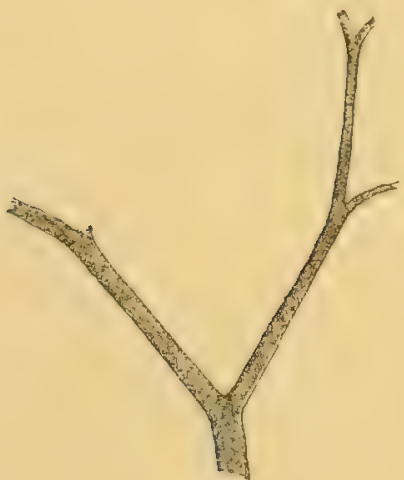

Iig. 44 .

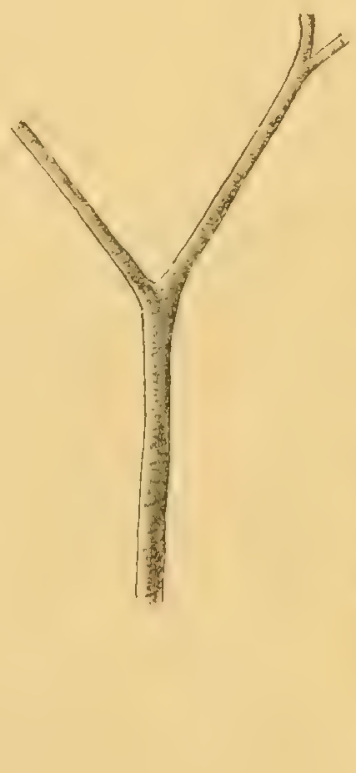

Fig. 12

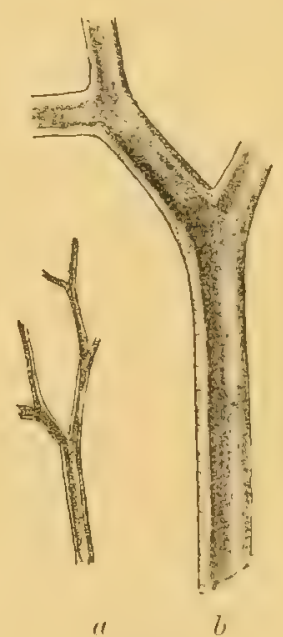

Fi⿺. 41 .

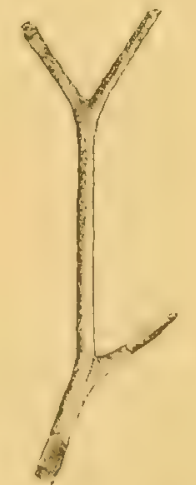

Fij. 4::

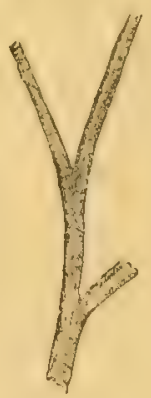

ligi. 45.

Fig. 41-46. IIostimella hostimensis. P. et B, $\alpha$ typica.

41-44. Srbsko. Dichotomies, rbourgeons" $\frac{1}{1}$

45. Chotec. Dichotomies $\frac{1}{1}$.

46. Choteč. Dichotomies, $a \frac{1}{1}, b \frac{3}{1}$.

2. $\beta$ ) rhoderformis, P. et B. Fig. 47-51.

Ce sont des restes généralement plus grands que notre forme typica, ramifiés en dichotomies souvent répétées, et dont les ramifications de plus en plus fines se terminent par des extrémités fréquemment un peu recourbées (fig. 47,50). Nous avons trouvé plusieurs échantillons semblables; nous en avons parlé à propos de notre Rhodea (voir pages 13,14 ), et nous avons signalé déjà les travaux et les opinions de Solms à ce sujet. Cet auteur a décrit une forme qui rappelle de très près nos échantillons, et qui, par ses dichotomies souvent répétées, par ses extrémités un peu recourbées, ferait penser aux Rhodea. Nous ne voulons pas affirmer cependant la parenté de nos empreintes avec les restes que nous avons décrits ci-dessus comme Spiropteris et Rhodea (?), mais nous voulons indiquer seulement que cette parenté ne doit pas être repoussée a priori. Il est regrettable que, par suite de l'allochtonie, les extrémités de ces branches délicates semblent être toujours cassées. Les petites frondes que nous avons décrites pourraient n'être que les extrémités des ramifications du type qui nous occupe. Cependant, comme il se pourrait aussi que ce fut autre chose, nous n'avons pas voulu les ranger dans la même catégorie et nous ne leur donnons pas le même nom. Afin de faciliter la discussion et pour qu'on puisse en parler, nous avons nommé notre 


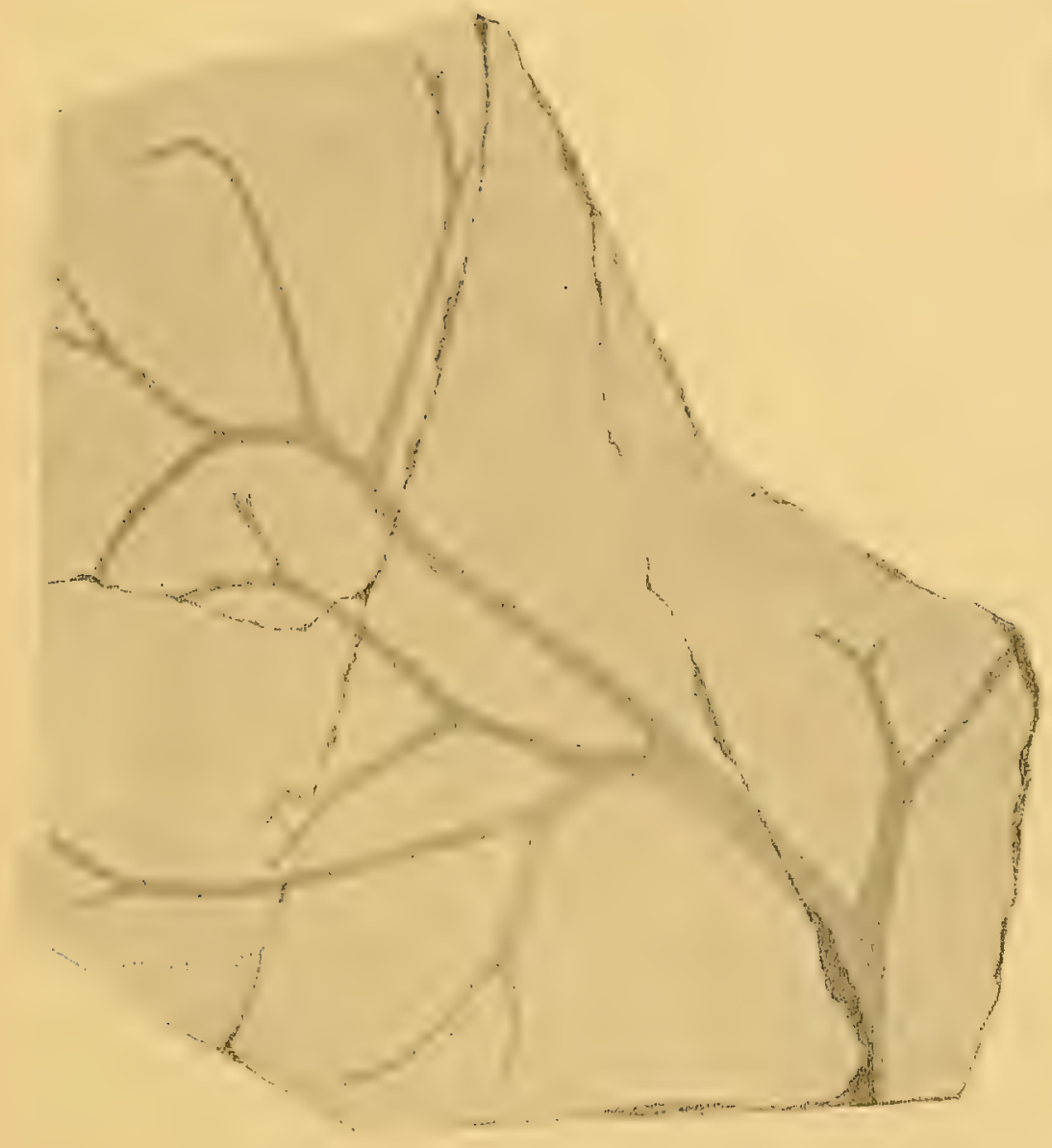

Fig. $47 . a$

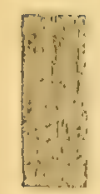

Fig. 47.7

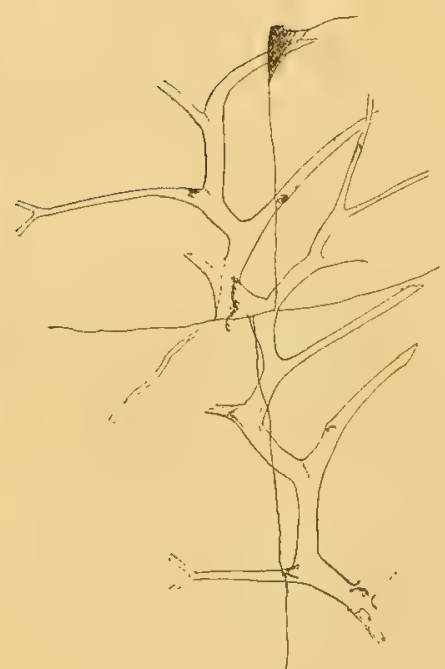

Fig. 49.

Fig. 47-49. Hostimella hostimensis, P. et B. B) rhodeaeformis.

47. Hostim. (K. k. geol. Reichsanstalt.) a) Ramifications nombreuses $\frac{1}{2}$, b) moutre les points à la surface $\frac{1}{1}$.

48. Hostim. (K. k. geol. Reichsanstalt.) Ramifications nombreuses $\frac{1}{4}$.

49. Srbsko. (Hofmuseum, Wien.) Ramifications nombreuses, "bourgeons"? $\frac{1}{2}$.

type Hostimella Tostimensis, $\boldsymbol{\beta}$ ) rhodecformis, de façon à n'affirmer en aucune manière une parenté avec un genre connu.

Nous avons pu voir parfois sur certains rameaux des espèces de cicatrices rappelant les „bourgeons" que nous avons décrits ci-dessus à propos de notre forme $\alpha$ ) typica et ces "bourgeons" nous ont permis d'établir un rapport entre les deux types. Il se peut que, si les "bourgeons" sont moins nombreux ici, c'est justement par le fait que les

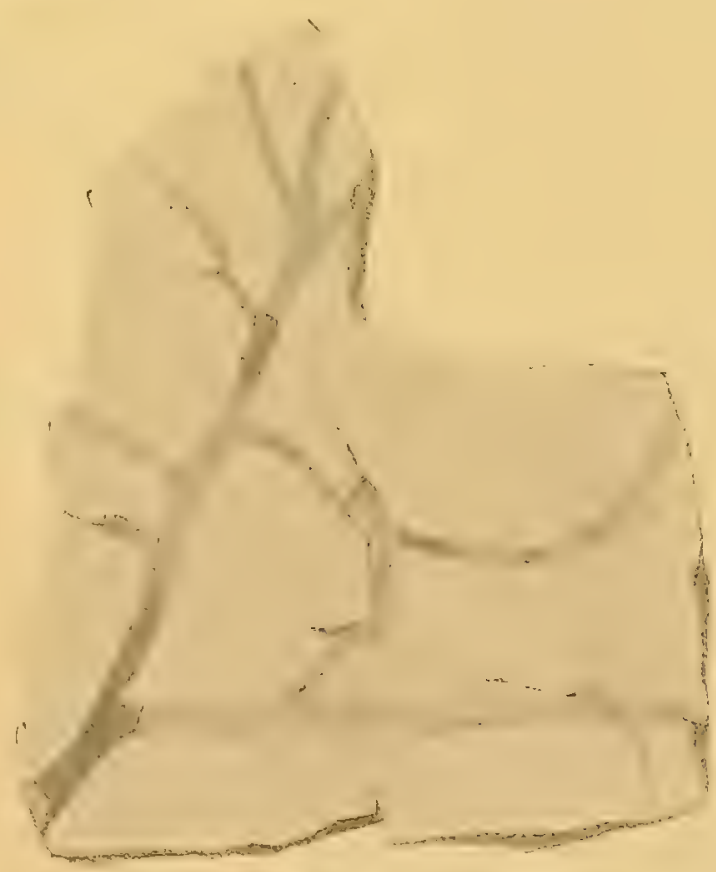

Fig. 48. 


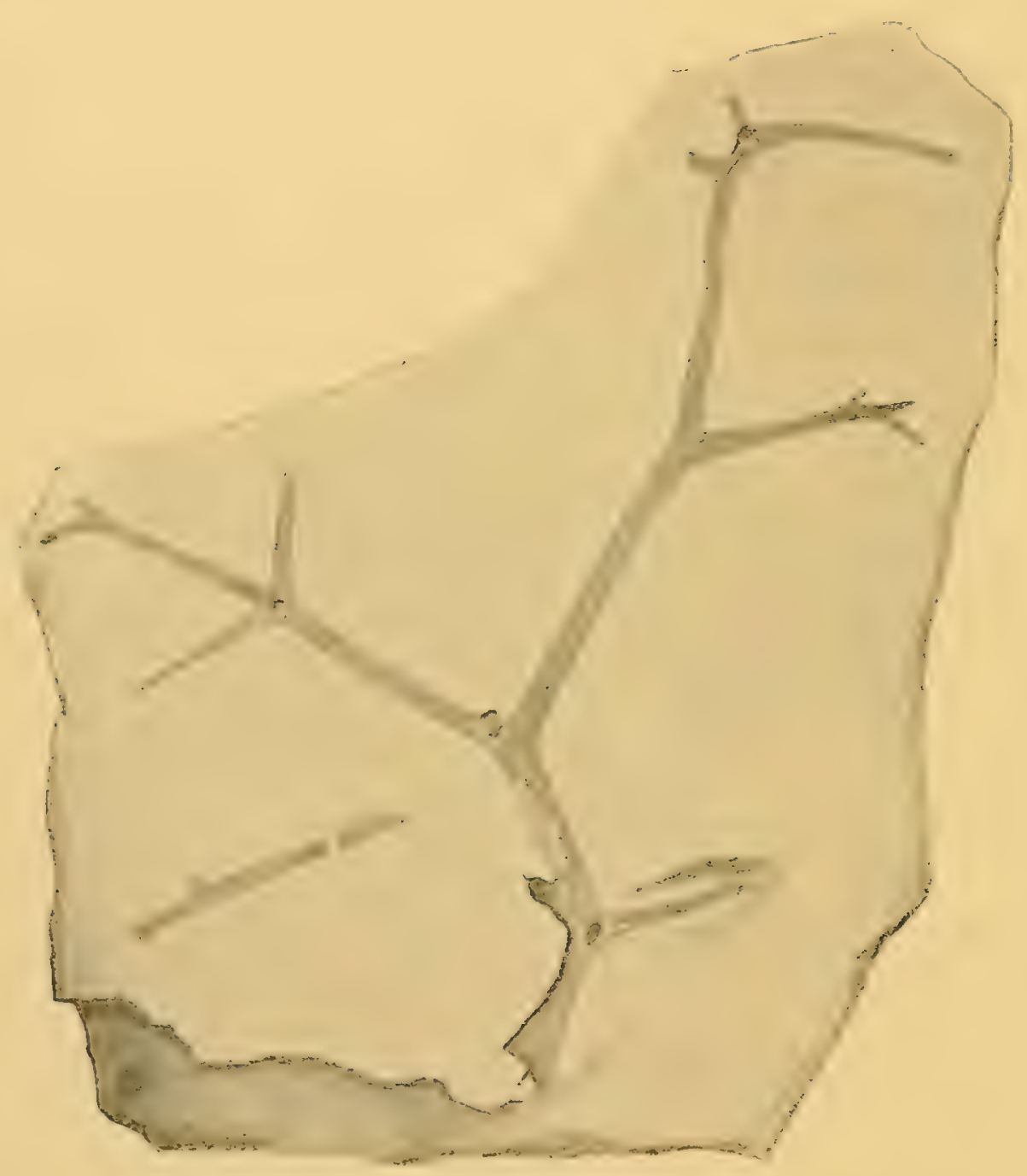

Fig. 50. Hostimella hostimensis, P. et B. $\beta$ ) rhodeaeformis.

50. Hostim. (K. k. geol. Reichsanstalt.) Ramifirations, "bourgeons" $\frac{1}{y}$. ramifications sont beaucoup plus abondantes. Nous arous pu constater aussi (fig. 476 ) les petits points à la surface de l'empreinte.

Ces deux types de Hostimella hostimensis ont été décrits par les auteur's précédents sous les noms de Fucoides hostinensis, Barr. ex parte, Hostinella hostinensis, Barr. (in Stur) ex parte, Haliserites zonarioides, Kr. ex parte.

Stur, dans les figures qu'il donne, n'a pas clairement représenté les détails de ces échantillons. Il s'en tient au nom cle Barrande et les considère comme des Algues. Quant ì nous, nous ne pouvons que répéter que nous ne savous oir les placer. A cause des "bour. geons" qu'on relicontre à la fois dans nos formes $\alpha$ et $\beta$, et à cause de l'analogie de la forme rhoderformis avec les Rhodea, nous pour-

rions peut-être y voir une parenté avec ce genre. D'autre part, nos deux types ne peuvent se séparer absolument, car certaines de nos figures montrent à la fois les caractères des formes $\alpha$ et $\beta$ : ramifications nombreuses (fig. 13 et 39), un peu courbées (fig. 20), „bourgeons“ bien nets (fig. 49 et 50 ). Les fig. 49 et 37 présentent entre elles de nombreuses analogies.

Nous devons relever en outre, un point qui pourrait venir à l'appui de ce que nous disions, plus haut, du rapport de ce type avec notre Spiropleris. C'est que Nathorst dans ses terrains déroniens de l'Hle des Ours a signalé, sous le nom de Sphenopteridium Keilhaui, des formes très semblables aux nôtres, tant par la dichotomie des rameaux que par les points de la surface et par les „bourgeons" qui sont à l'angle des dichotomies. En outre Nathorst a été en possession des ramifications latérales portant les pinnules, pinnules qu'il dessine et qu'on peut reconnaitre très analogues aux $R$ hodea, comme nous l'avons déjà relevé. Nous n'avons pu constater de nervures ni sur les uns ni sur les autres de nos échantillons; c'est pour cela que nous ne pouvons prétendre que nous soyons en présence du Sphenopteridium, et nous préférons ne rien préciser. Nous n'avons jamais pu non plus 
trouver réunis les deux types Rhodea et Hostimella sur le même échantillon; mais puisque nous avons d'une part les extrémités des pinnules, d'autre part les axes, nous serions presque en droit de les rapporter les uns aux autres.

Sous le nom de Rhachiopteris tomistriata, Dawson a aécrit, dans sa ,Flora of the DevonPeriod", 1862/63, des axes avec des cicatrices rappelant certaines de nos formes de Hostimella.

Nous devons relever aussi le fait qu'un de nos Pscudosporochnus était accompagné d'un tel fragment dichotomique avec "bourgeon". Nous ne croyons pas cependant que notre Hostimella puisse se rapporter à notre Pseudosporochnus, car cette constatation était un fait purement isole; d'autre part, ce fragment dichotomique n'était pas directement réuni au Pseudosporochnus et pouvait être un débris ne se trouvant là que par hasard.

\section{Incertie sedis.}

Nous avons représenté dans la fig. 52 un type que nous ne savons pas à quoi rapporter; nous le plaçons ici, non pas qu'il soit possible de lui supposer un rapport quelconque avec Hostimella, mais parce que, sur cet axe et à l'aisselle d'appendices en forme de feuilles, il y a des "bourgeons“ ou du moins des espèces de marques arrondies qui pourraient faire penser aux bourgeons de Hostimella. La fig. 51 montre en effet en deux ou trois places des "bourgeons" dont la disposition rappelle vaguement celle des organes similaires de la fig. 52. Cette dernière représente du reste, un échantillon assez mal conservé que nous avons eu avec sa contre empreinte; il était en trop mauvais état pour mériter une dénomination spéciale, mais assez curieux pour être signalé.

\section{Cf. Asterocalnmires scrobiculatus, (Schloth.) Zeiller.}

Fig. 53.

Voir la synonymie dans Potonié: Protocalamariacées, p. 558 dans Engler \& Prantl, Ptéridophytes.

C'est le type Calamites transitionis des auteurs, suffisamment connu pour qu'il nous soit inutile d'en donner une nouvelle description. (Voir à ce sujet: Potonié dans Engler \& Prantl, $l$. $c$. et Potonié, Lehrbuch, p. 365-366, et Silurflora.) Ce dernier anteur disait dans sa "Silurflora", que Asterocalamites commençait avec le Culm et qu'on ne le connaissait pas encore certainement des étages précédents. Notre échantillon pourrait donc laisser croire que ce genre apparaîtrait déjà dans le Dévonien. Du reste, dans le même ouvrage, p. 66, l'auteur signale un reste qu'il considère comme semblable 


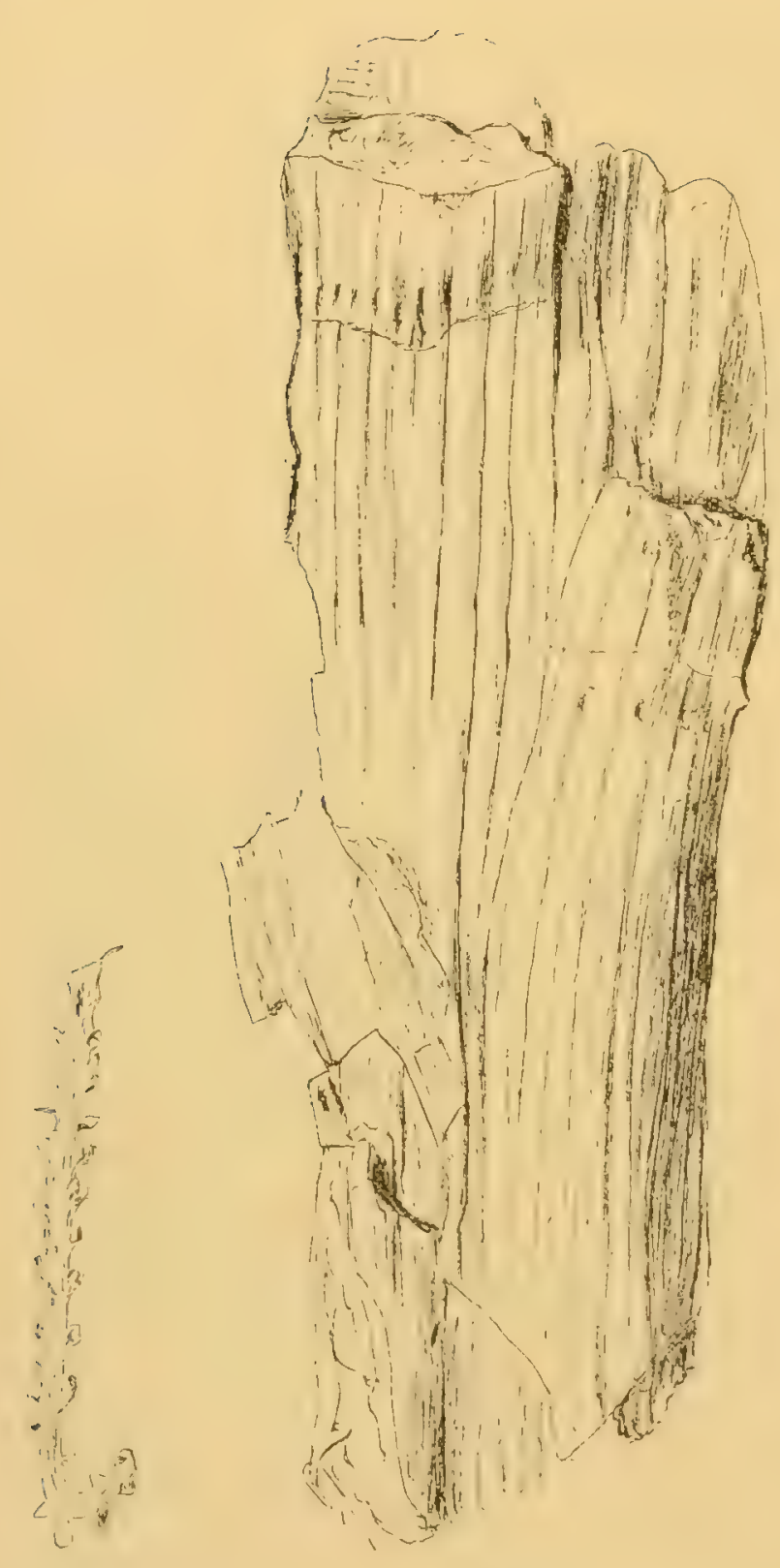

Fig. 52.

Fig. 53.

Fig. 52. Incertæe sedis.

52. Srbsko. (Geol. Landesmuseum, Berlin.) „Bourgeons"? feuilles? $\frac{1}{2}$.

Fig. 53. Cf. Asterocalamites scrobiculatus, (Schloth.) Zeiller.

5ร. Srbsko. (Collection de M. Dusl à Beraun.) $\frac{1}{1}$. acquis dans cet horizon sa plus grande extension, tandis que dans les étages précédents elle n'aurait fait que commencer son développement. Quant au nom, nous avons dit: Cf. Asterocalamites scrobiculatus, car nous ne voulons pas affirmer être en présence de cette espèce; les caractères, il est à Asterocalamites; ce serait donc une première apparition de ce genre lans des terrains cncore beaucoup plus anciens que ceux qui nous occupent et où nous sigualons sa présence probable.

Liuique échantillon que nous rattachons ¿ cette espèce, et provenant de la région dévonienne du centre de la Bohême nous est arrivé avcc sa contreempreinte, par l'intermédiaire de M. I)usl, mais il est malheureusement trop mal conservé pour que nous soyons autorisés à le déterminer certainement et définitivement comme appartenant à la même espèce que les pièces si caractérisées du Culm (Potonié). Mais on peut roir assez clairement les caractères de l'espèce.

Si c'est un Asterocalamites, nous n'avons, comme c'est presque toujours le cas, que le moulage du centre de la tige; les stries sont en continuation et non en alternance d'un entrenœud à l'autre; puis aux noeuds, comme nous n'avons pas les lignes transversales, nous avons pu constater en deux endroits, et de façon assez nette, les points nodiaux décrits Culm (p. ex. Polonié, Silurflora, fig. 49, p. 89). Il est un point à relever, c'est que notre échantillon se présente comme un axe dichotomisé. Or on sait que chez Asterocalamites, qui est la plus vieille des Equisetales connues, les feuilles se divisaient dichotomiquement. On peut supposer que, chez des échantillons du Dévonien, plus anciens done que ceux du Culm, la dichotomie puisse se retrouver encore dans le tronc, tandis quelle naurait persisté daus la suite que chez les feuilles. Nous avons, dans le cours de ce travail, assez souvent l'occasion de signaler cette persistance des caractères ancestraux chez les feuilles jour qu'il soit inutile de s'y arrêter longuement ici. Que ce fossile ne nous soit parvenu du terrain de Bohême qu'à l'état d'un seul échantillon, cela n'a rien non plus qui doive nous étonner: si cette plante est fréquente dans Je Culm, c'est qu'elle aurait déjà chez Asterocalamites scrobiculatus du 
vrai, semblent concorder, mais nous trouvons les stries moins nettes; labsence de données très aftirmatives ne nous permet pas de dire à coup sûr que nous pouvons ranger ce fossile sous ce nom spécifique, ou lui en attribuer un autre.

\section{Pacudosporocinne Frejèí, (Stur ex parte) P. et B.}

Fig. $5.1-81$.

Chondrites verticillatus, Kr.

Sporochnus Krejecii, stux ex parte.

Hostinella hostinensis, Barr. (in Stur) ex parte.

? Barrandites, Ettingsh. (in Stur) ex parte.*)

Nous avons eu la chance de trouver suffisamment d'échantillons, et de suffisamment bien con. servés pour pouvoir recoustituer dans ses parties les plus essentielles une plante de nos terrains de Bohême.

Nous répétons ce que nous avons dit plus haut à propos de nos restes de Fougères, que nous ne comprenons pas pourquoi Stur a séparé dans deux espèces et dans deux genres différents des échantillons qui nous sont clairement apparus comme ayant appartenu à des plantes semblables.

Conme nous le verrons plus loin, les auteurs ont eu tort, selon nous, de considérer cette plante comme une Algue. Nous sommes empêchés de conserver un des noms de genre qu'ils lui ont donné, puisque ce nom de Sporochmus est appliqué à une Algue vivante. Aussi, nous avons adopté le nom de. Pseudosporochnus, espèce $P$. Krejčii. Les échantillons que nous allons décrire ont été trouvés dans les stations de Hostím et Srbsko.

1. Nous sommes en présence d'une part (fig. 54-58,63-69, photographie, fig. 81), de troncs assez grands, pouvant atteindre $40^{\mathrm{cm}}$ et plus $\left(2^{\mathrm{m}}\right)$ de hauteur, sur 2 à $4^{\mathrm{cm}}$ et plus $\left(20^{\mathrm{cm}}\right.$ ) de largeur, selon la hauteur considérée. A la base, ces trones vont s'élargissant régulièrement comme un cône qui aurait été aplati (fig. 57, 58, 67-70). Dans le cas représenté par notra photographie, fig. 81, nous voyons encore le tronc entier, et nous remarquons que la base a plus ou moins la forme d'un bulbe.

Au sommet, les branches sont divisées en plusieurs rameaux. Les branches et les rameaux sont dûs à une ramification dichotomique, du tronc d'abord (fig. 61, 76), puis qui se répète sur les ramifications ou sur une des ramifications, et cela si rapidement quil en résulte pour le sommet du tronc une ramification en apparence tri-tétra-penta-palmée (fig. 55-58, 62, 63, 69, 82). Plusieurs de nos pièces prouvaient très nettement cette interprétation, et l'on voyait la dichotomie des branches naître presque à la même hauteur que la dichotomie du tronc, mais cependant un peu plus haut de façon à montrer clairement de quoi il s'agit (fig. 55, 57,60,63, 64, 67, 68, 70). 'Très souvent nous avons pu voir les ramifications dichotomiques disposées comme en plusieurs étages (fig. 63, 68, 69). Un autre caractère à sigualer est la présence à la base des échantillons, partant de la partie élargie, et sur une hauteur plus ou moins grande, dappendices (racines?) qui servaient en tous cas à la fixation de la plante au sol (fig. 67, 69, photographic, fig. 81). Ces appendices sont quelquefois en touffes très serrées, peu claires.

Le plus souvent on trouve sur les troncs (fig. 54, 56, 62, 63, 66, 67, 82) et sur les branches (fig. 56. 70) des marques**) knorrioïdes; les Knorria ne sont autre chose, comme on le sait,

*) Voir note $* * *)$ p. 11.

**) Traduction du mot allemand "MIal", les cicatrices étant des ${ }_{2} N a r b e{ }^{4}$. (Ne pas oublier qu'une cicatrice indique généralement l'endroit où se trouvait un organe qui est tombé, tandis que l'expression "MIal" peut se rapporter à toutes sortes de marques de différentes natures et que souvent on ne sait comment interpréter.) 
que les moulages subcorticaux des tissus parenchymateux (parychnos) qui entouraient les traces foliaires des Lépidodendracées ei des Bothrodendracées. Notre type rappelle le plus Knorria acicularis des Bothrodculracées, qui se caractérise par des bourrelets knorrioïdes (Kinorriawulst) longs et minces et assez espacés. Ces Knorria, s'atténuant peu à peu, peuvent se prolonger assez bas le long du tronc, se disposer de façon plus ou moins régulièrement spiralée; elles ont généralement

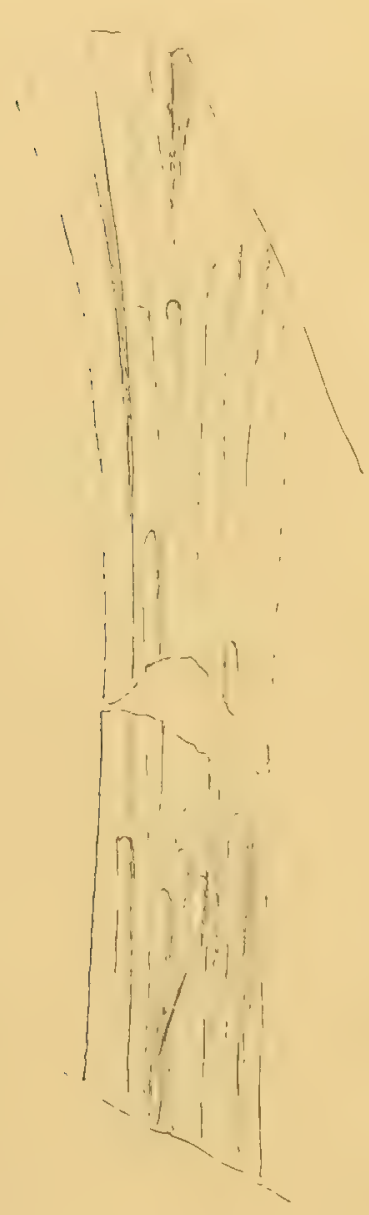

Fig. 54.

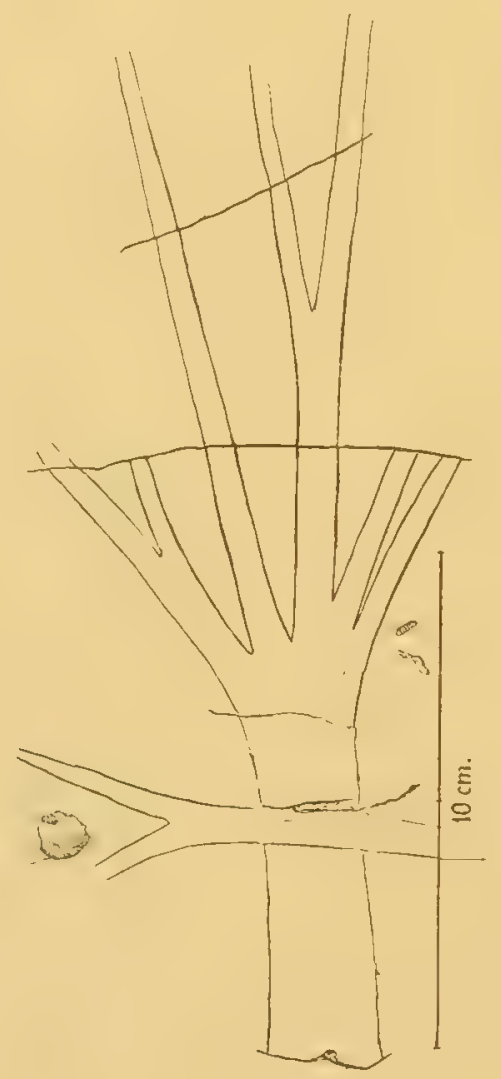

Fig. 55 .

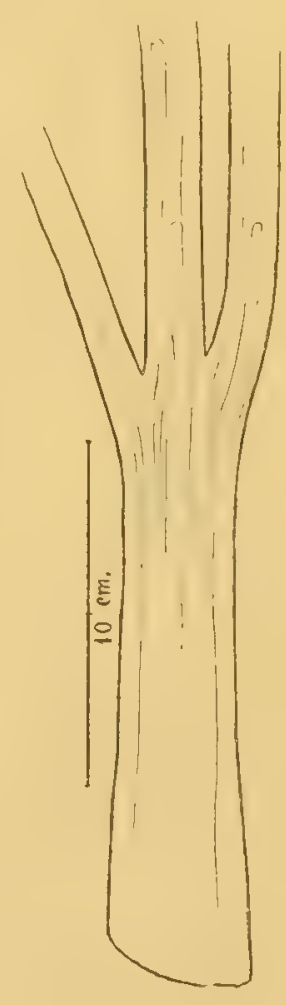

Fig. 56 .

Fig. 51-56. Pseudosporochnns Krejěii, (Stur ex parte) P. et B.

54. Hostim. (Univ. Wien) Surface knorrioïde $\frac{1}{1}$.

55. Srbsko. (Univ. Wien.) Dichotomie en éventail $\frac{1}{2}$

56. Srusko. (K. k, Uaiv. Prag.) Trichotomie, Kinorria $\frac{1}{3}$.

$2^{m m}$ de largeur. Nathorst, dans son "Ile des Ours" et Potonié dans sa "Silurflora", signalent le fait que souvent des Knorria acicularis ont été décrites comme Astcrocalamites, et Potonié les désigne comme $K$. acicularis calamitoilde". Un cas semblable se rencontre chez notre Psendosporochmus: chez cette plante, nous avons généralement des Knorria, mais nous avons constaté (fig. 65) des Knorria si allongées, si étroites et si rapprochées les unes des autres qu'elles donnent l'apparence générale d'une Calamitéc. 
Quelquefois les Knorria font défaut, mais on voit sur le tronc de petites traces (fig. 58,63, 64) comme des lignes très fines, plus ou moins longues, toutes dirigées dans le sens longitudinal du tronc ou des branches, et qui pourraient être les impressions des rayons médullaires d'un bois, car

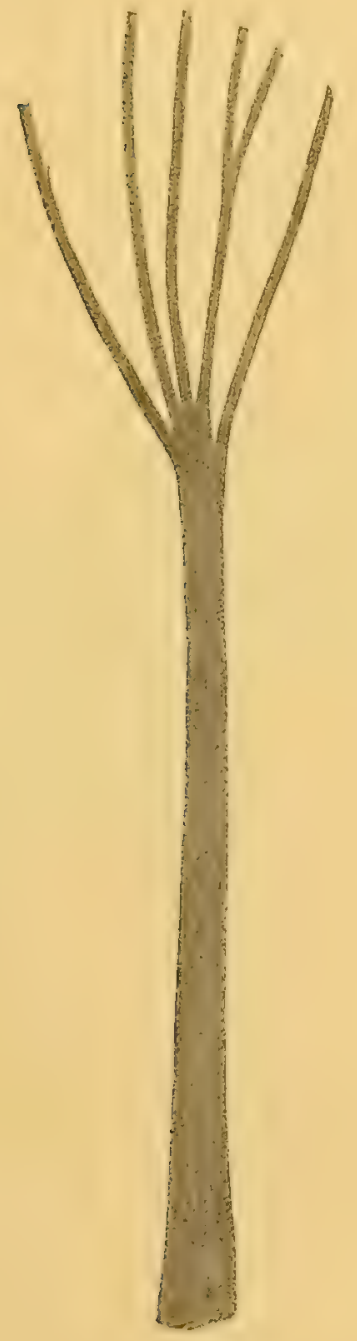

Fig. 57.

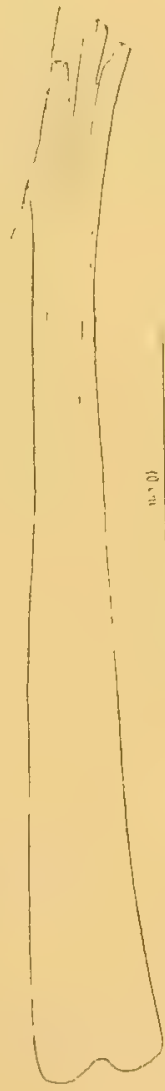

Fig. 58

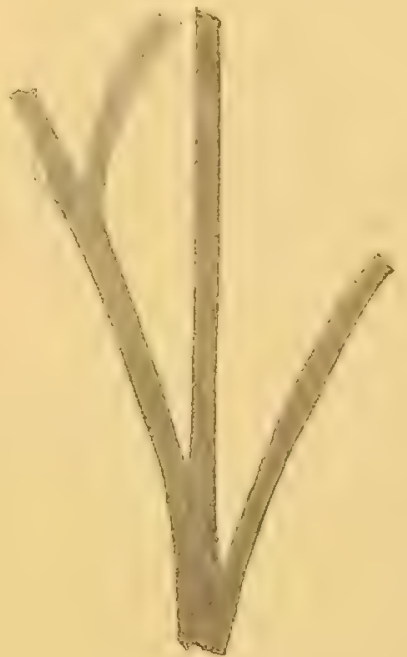

I'ig. 60

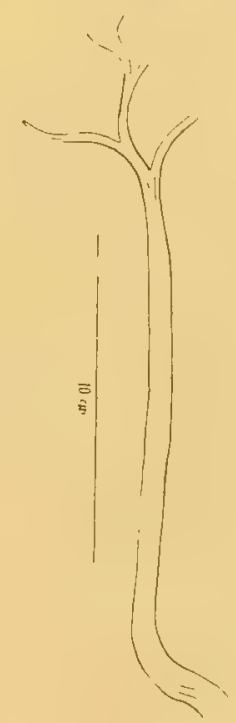

Fig. 59 .

Fig. 57-60. Pseudosporochnus Krejěii, (Stur ex parte) P. et B.

57. Dichotomie en éventail $\frac{1}{3}$

58. Hostim. (Geol. Reichsanstalt, Wien.) Troncs élargis à la base $\frac{1}{3}$.

59. Hlubočep. (Deutsche Univ. Prag.) Dichotomie $\frac{1}{3}$.

60. Dichotomie $\frac{1}{2}$.

nous verrons plus loin que nous sommes ici en présence de plantes ayant possédé un bois bien caractérisé

Nous avons trouvé à Srbsko, dans les fouilles que nous avons fait faire, une pièce de 2 mètres de hauteur environ, composée d'un tronc de $20^{\mathrm{cm}}$ de large à la base et sarrêtant en haut aux pre- 
mières ramifications qui n'étaient malheureusement conservées que sur une très faible longueur. Nous avons fait photographier (fig. 81) cet échantillon intéressant qui prouve donc que notre Pseudo-

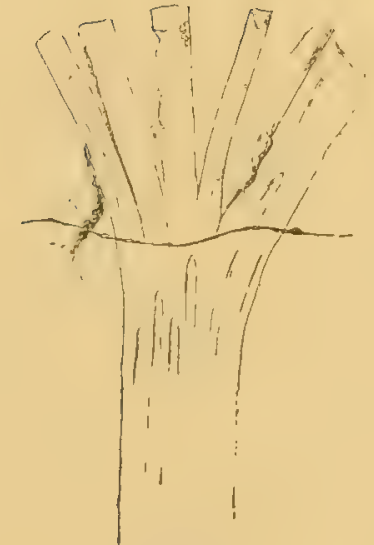

Fir. 6으.

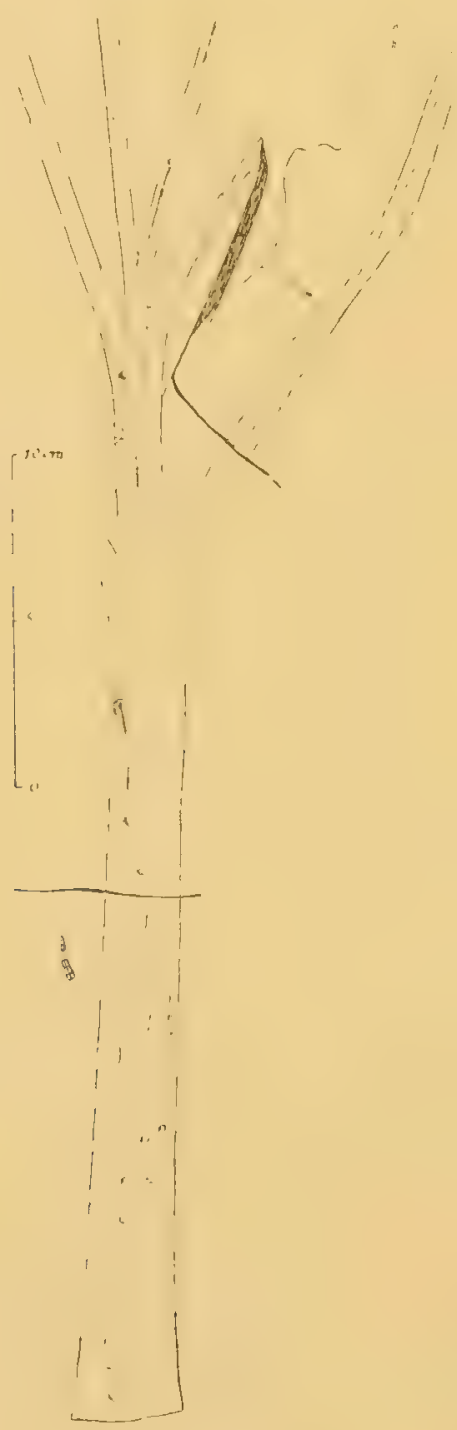

Fig. 63.

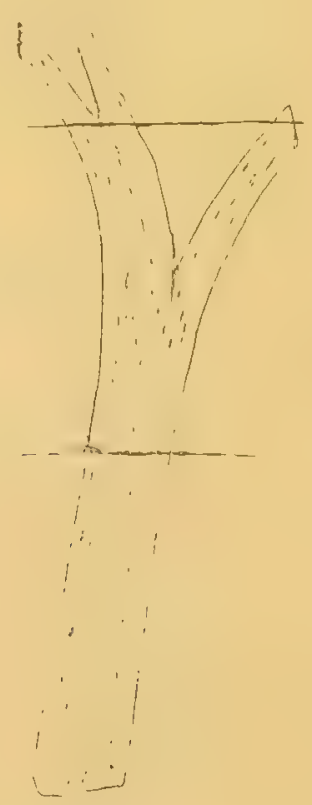

Fig. 61.

Fig. 61-64. Pseudosporochnus Krejěii, (Stur ex parte) P. et $\mathbf{B}$.

61. Srbsko. (Reichsanstalt, Wien.) Dichotomie. Elargissement basilaire $\frac{1}{5}$.

62. Srbslio. (Univ. Wien.) Dichotomie en éventail: Kunorria $\frac{1}{2}$.

63. Srbsko. (Univ. Wien.) Graptolithe à gauche Ramifications en deux étages. Kinorria $\frac{1}{3}$.

61. Srbsko. (Univ. Wien.) Stries longitudinales à la surface $\frac{1}{2}$.

sporochnus pouvait atteindre la hauteur d'un petit arbre. Ce type de fossiles, ainsi que ceux que nous allons décrire, sont certainement les empreintes végétales les plus abondantes des stations qui nous occupent. 


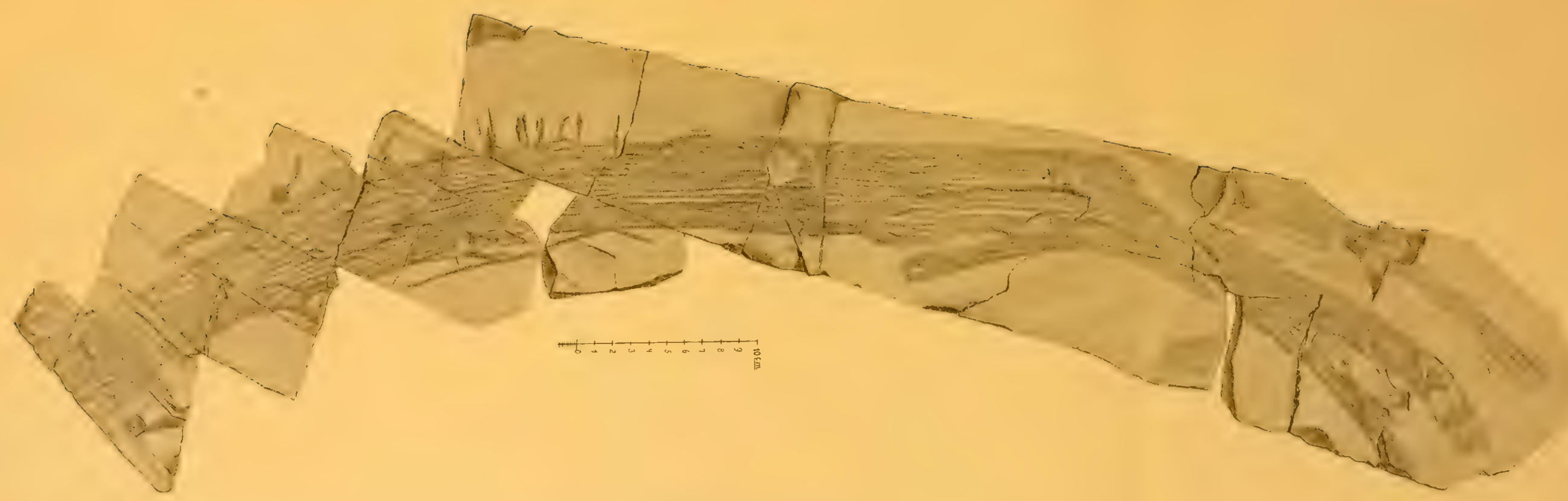

Fig. 65. Pseudosporochnus Krejèii, (Stur ex parte) P. et B.

65. Srbsko. (Reichsanstalt, Wien.) Stries longitudinales, apparence calamitoïde, appendices latéraux $\frac{1}{3}$. 



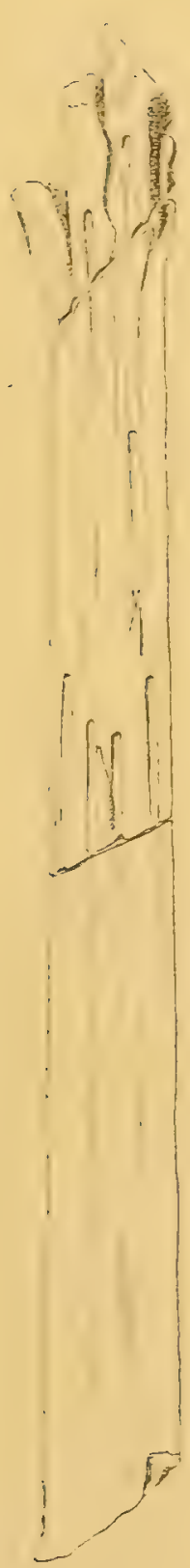

Fig. 6 h.

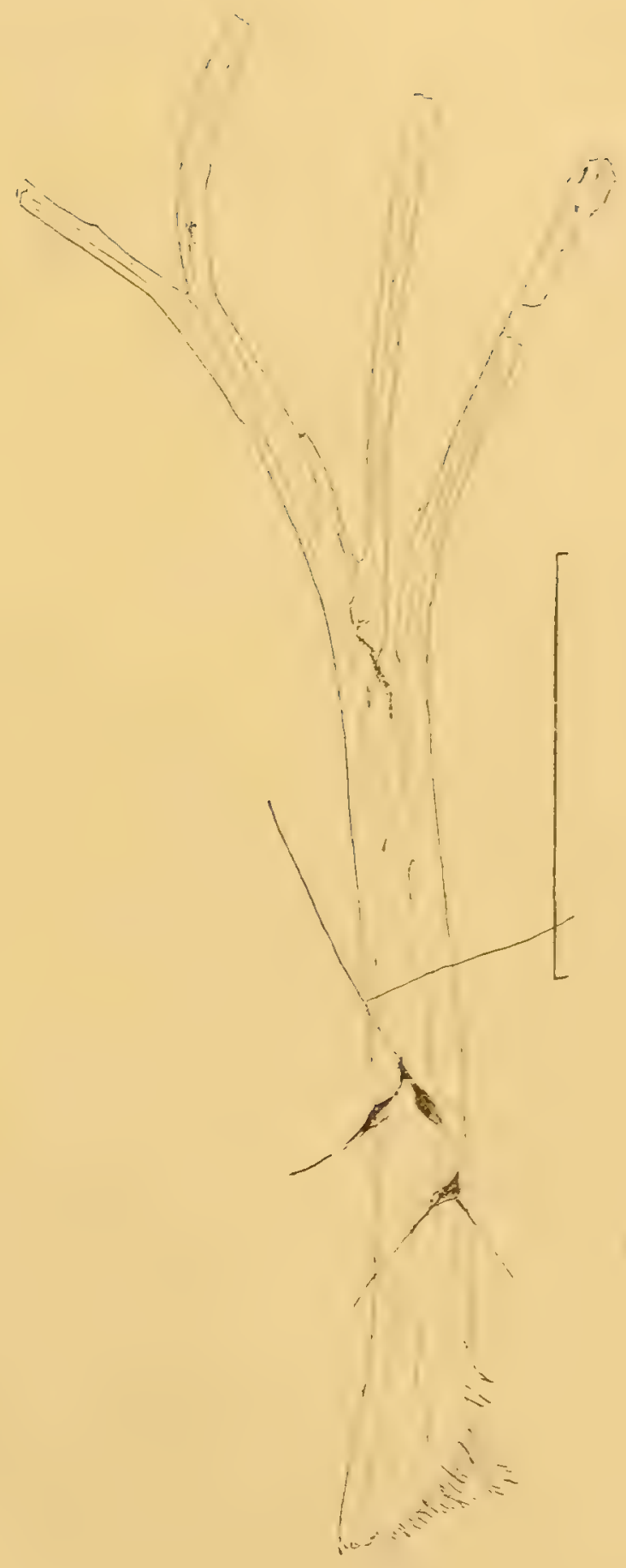

Fig. 67.

Fir. 63 .

Fig. 66-68. Pseudosporochnus Krejěí, (Stur ex parte) P. et B.

66. Srbstio. (Unir. Wien.) Knorria $\frac{1}{2}$.

67. - (Collection de Mr. Dusl à Beraun.) Original de Stur, Pl. III, fig. 2. Dichotomies, Knorria, appendices basilaires (racines?) $\frac{1}{2}$.

68. (Geol. Landesmuseum, Berlin.) Stries longitudinales, ramifications en denx étages, Elargissement basilaire $\frac{1}{6}$. 
2. D'autre part (fig. $71-80$ ), nous avons pu trouver des branches qui partaient parfois d'une base ramifiée en simple ou double dichotomie et qui se ramifiaient à leur tour en un grand nombre

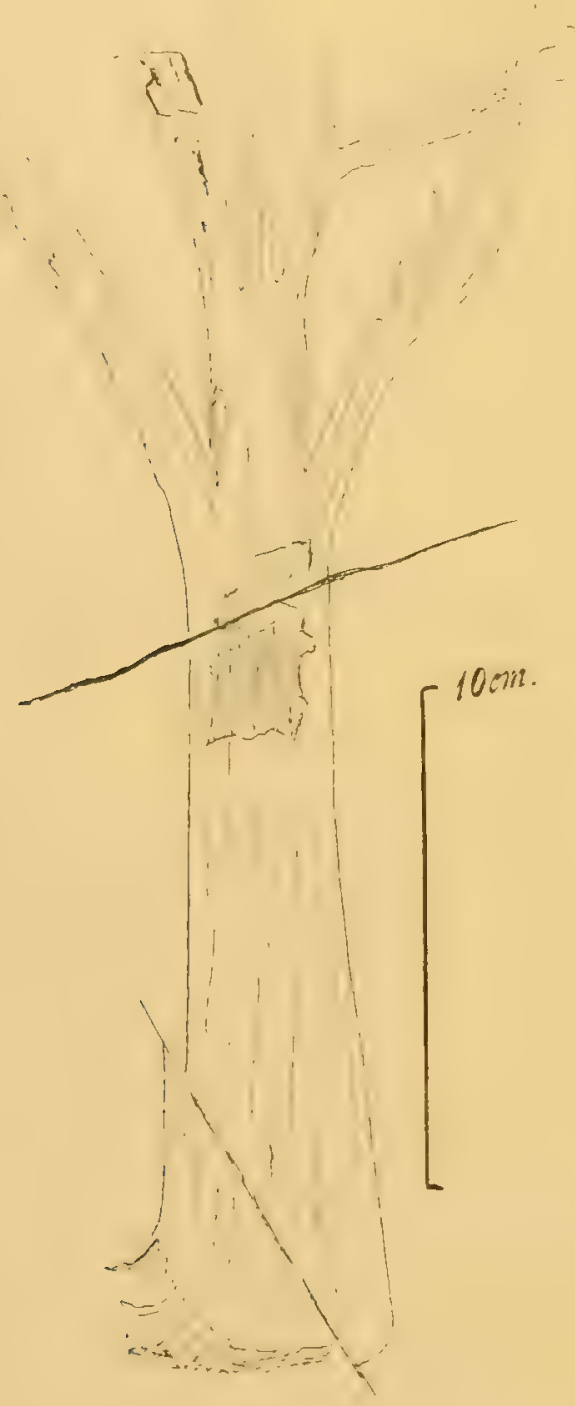

Fig. 69.

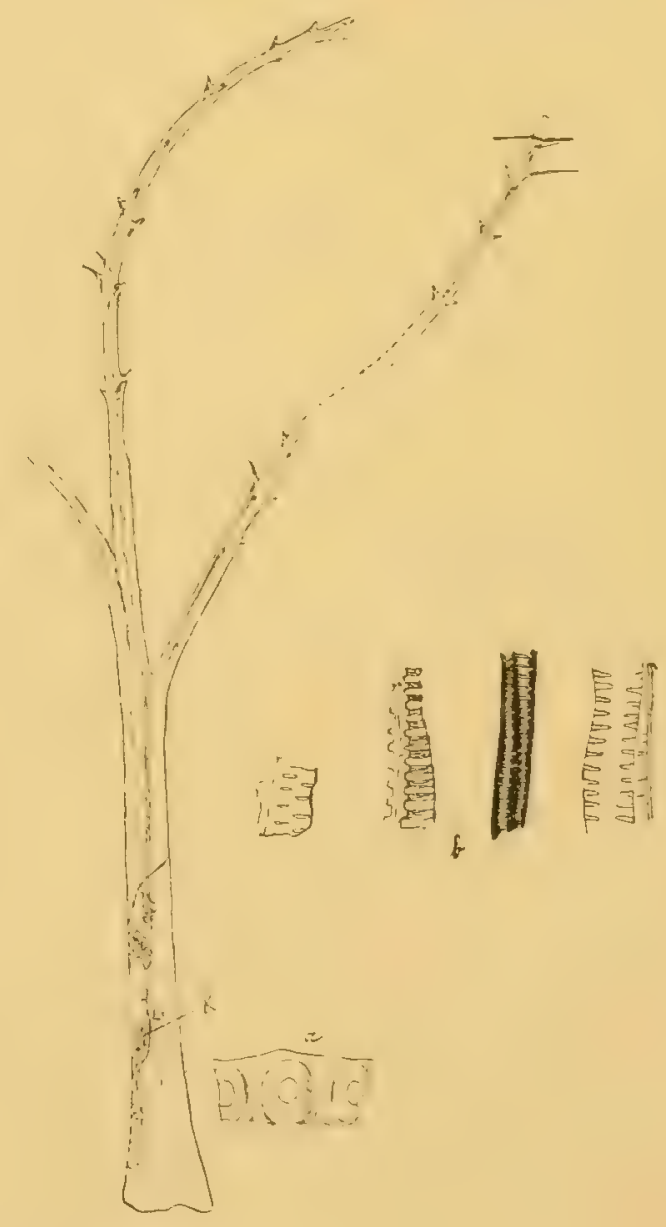

Fig. 70 .

Fig. 69-70. Pseudosporochnus Krejěii, (Stur ex parte) P. et B.

69. Srbsko. (Collection de M. Dusl à Beraun.) Original de Stur Pl. II, fig. 4. Dichotomies, appendices basilaires (racines?) $\frac{1}{2}$.

70. Srbsko. (Hofmuseum, Wien.) Knorria, élargissement basilaire, appendices latéraux (ramifications cassées) $\frac{1}{2}$. La macération de la houille trouvée à la surface $(K)$ a donné $a$ ) des ponctuations aréolées, $b$ ) des hydrostéréides de diverses natures 3.50 .

de ramifications. Celles-ci sont pennées, mais dérivent d'une dichotomie dont une des branches filles prendrait la direction principale, rejetant la branche sœur de côté, de façon à former un sympode 


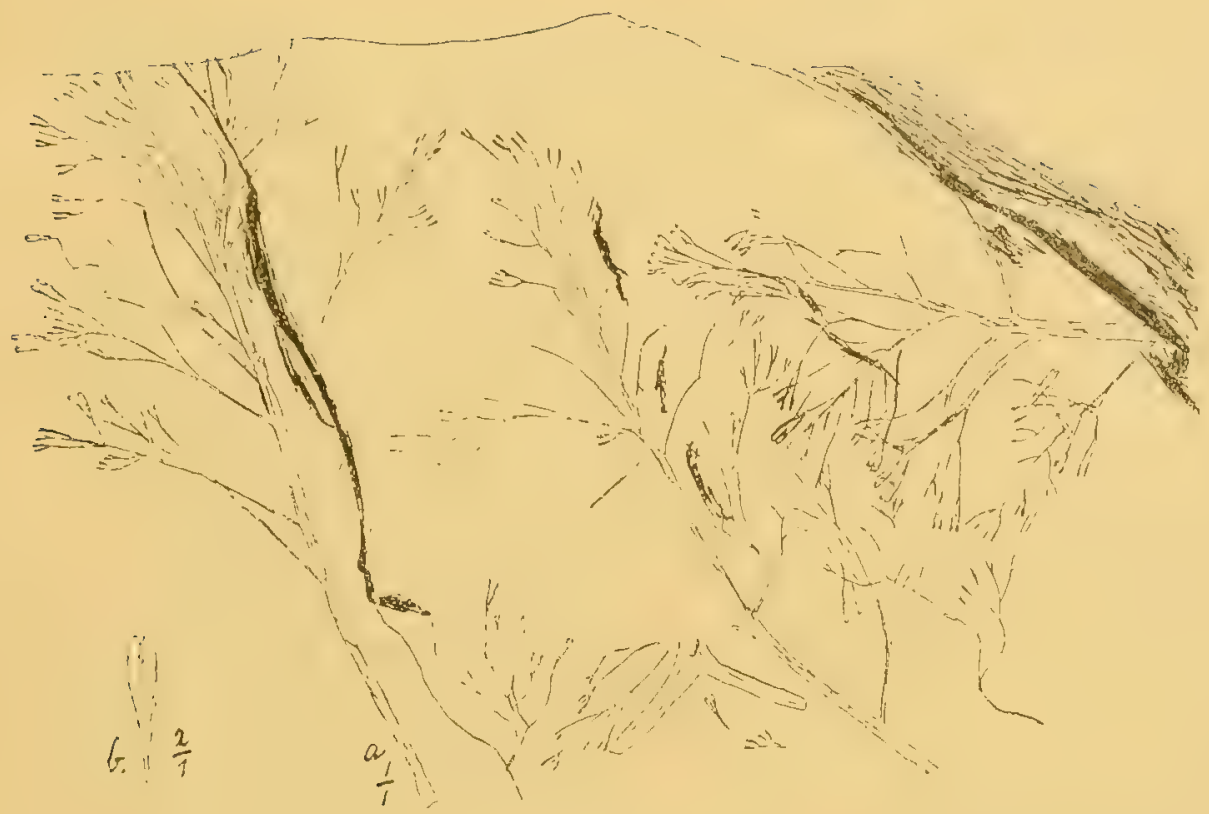

Fig. 71.

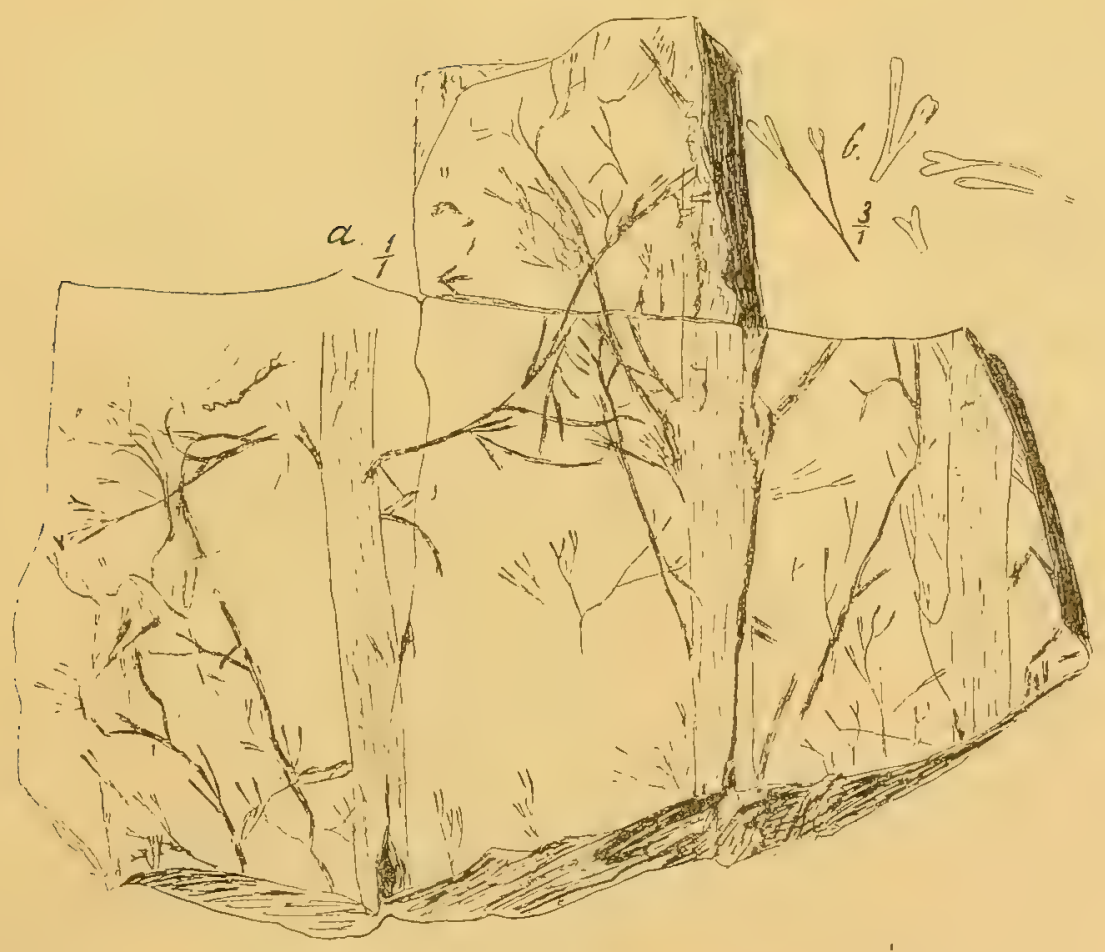

Fig. 72.

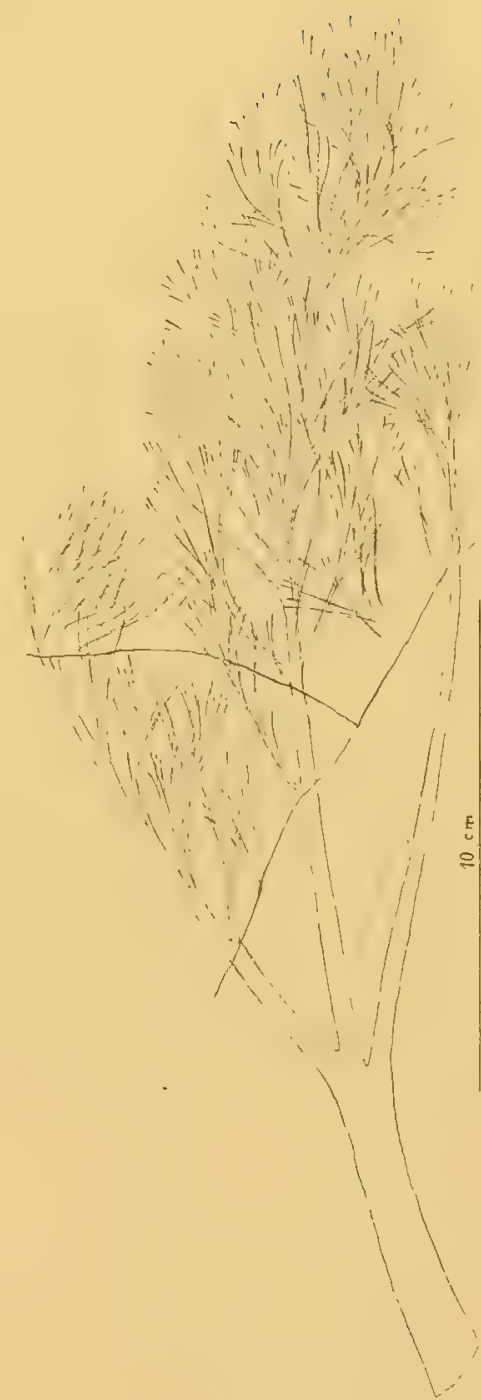

Fig. 73.

Fig. 71-73. Psendosporochnus Krejčii, (Stur ex parte) $P$, et B.

71. Srbsko, (Collection Dusl.) Original de Stur: Pl. II, fig. 2, a) Ultimes ramifications renflées $\frac{1}{1}, b$ ) une de ces ramifications latérales ayant l'air de se diviser en deux moitiés $\frac{3}{1}$.

72. Srbsko. (Geol. Landesmuseum, Berlin.) a) comme ci-dessus $\frac{1}{1}, b$ ) divers stades d'écartement des terminaisons (sporanges?) 3 .

73. Srbsko. (Böhm. Univ. Prag.) Original de Stur: PI. II, fig. 1. Dichotomies; renflements des terminaisons ultimes $\frac{1}{2}$. 
dichotomique. Tout serait donc divisé sur le mode dichotome. Les rameaux eux-mêmes se divisent à leur tour en dichotomies plus ou moins régulières, en branchettes très fines et dont les extrémités ultimes sont renflées, parfois assez fortement (fig. 71, 72, 73, 77, 79, 80). Ces extrémités étaient souvent curieusement dichotomes; nous les avons représentées, en les grossissant 2 ou 3 fois, dlans les fig. 71 et 72 ; on pourrait les considerer daus ces dessins comme divers moments de l'ouverture de sporanges (si, comme nous le discuterons plus bas, ce sont des sporanges).

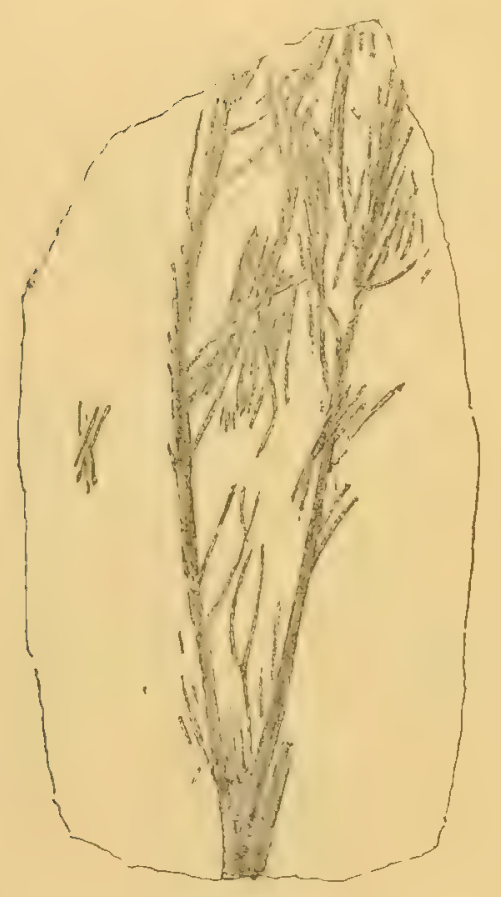

Fiv. 75 .

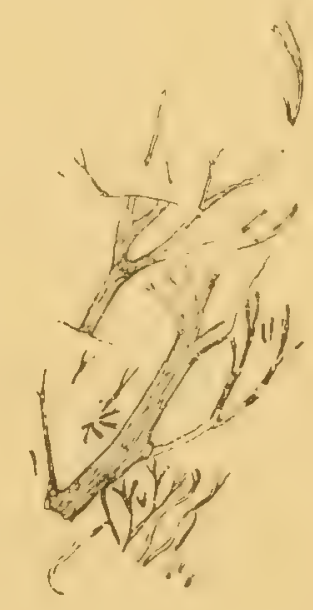

Irig. 71

Fig. 74-75. Psendosporochnus Kirejéi, (Stur ex parte) P. et $\mathrm{B}$.

74. Srlstio. (K. k. geol. Reichsanstalt.) Ramifications; infiltrations minérales $\frac{1}{T}$.

75. Srbsko. Infiltrations minérales $\frac{1}{1}$.

débris mieux caractérisés, dont nous venons de donner l'apparence. Le tronc élargi en bas, la ramification deux fois dichotomique du sommet nous avait donné l'ilée de la parenté avec les plantes ci-dessus; mais notre conception était troublée par la présence sur les branches de petits appendices que nous prenions pour des feuilles et que nous ne pouvions rencontrer dans les autres types. En regardant plus attentivement, nous avons vu qué ces appendices étaient de longueurs différentes et comme cassés au sommet; nous avons alors compris quils pouvaicnt être les débris des fines ramifications que nous venons de décrire et qui auraient été cassées près de la branche qui les portait. Des Knorria se trouvaient sur les branches et semblaient être les marques sous-corticales sinon de feuilles, du moins de ces petites ramifications. Sur le tronc, les Knorria pourraient être les traces de feuilles qui auraient été disposées en spirale; mais nous ne pouvons que formuler une supposition à cet égard, car nous n'avons pas pu découvirir les feuilles en question. Souvent ces empreintes 


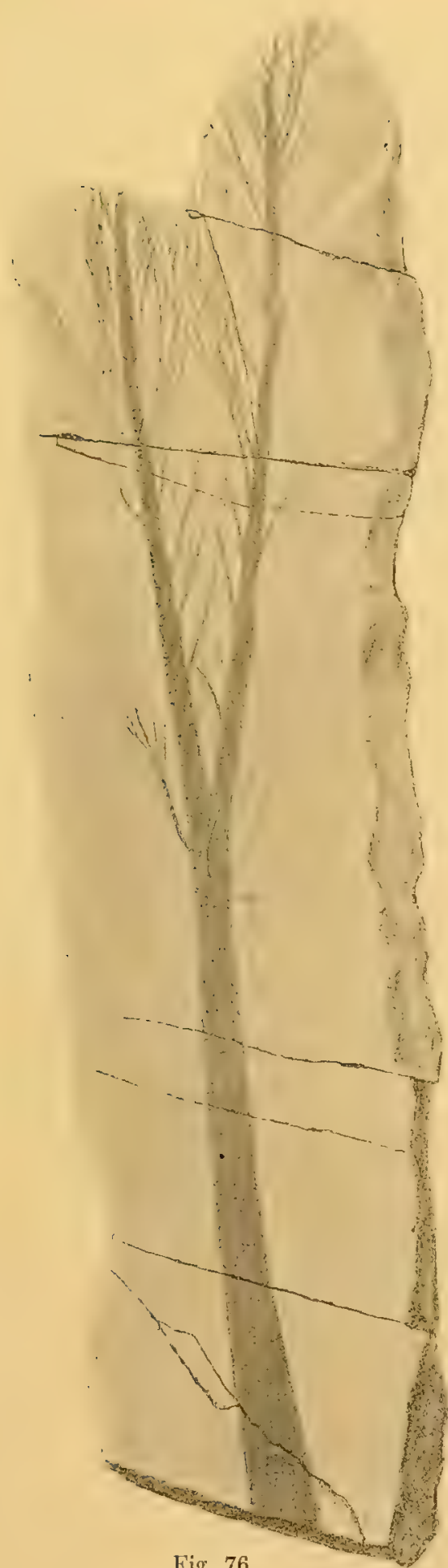

Fig. 76 .

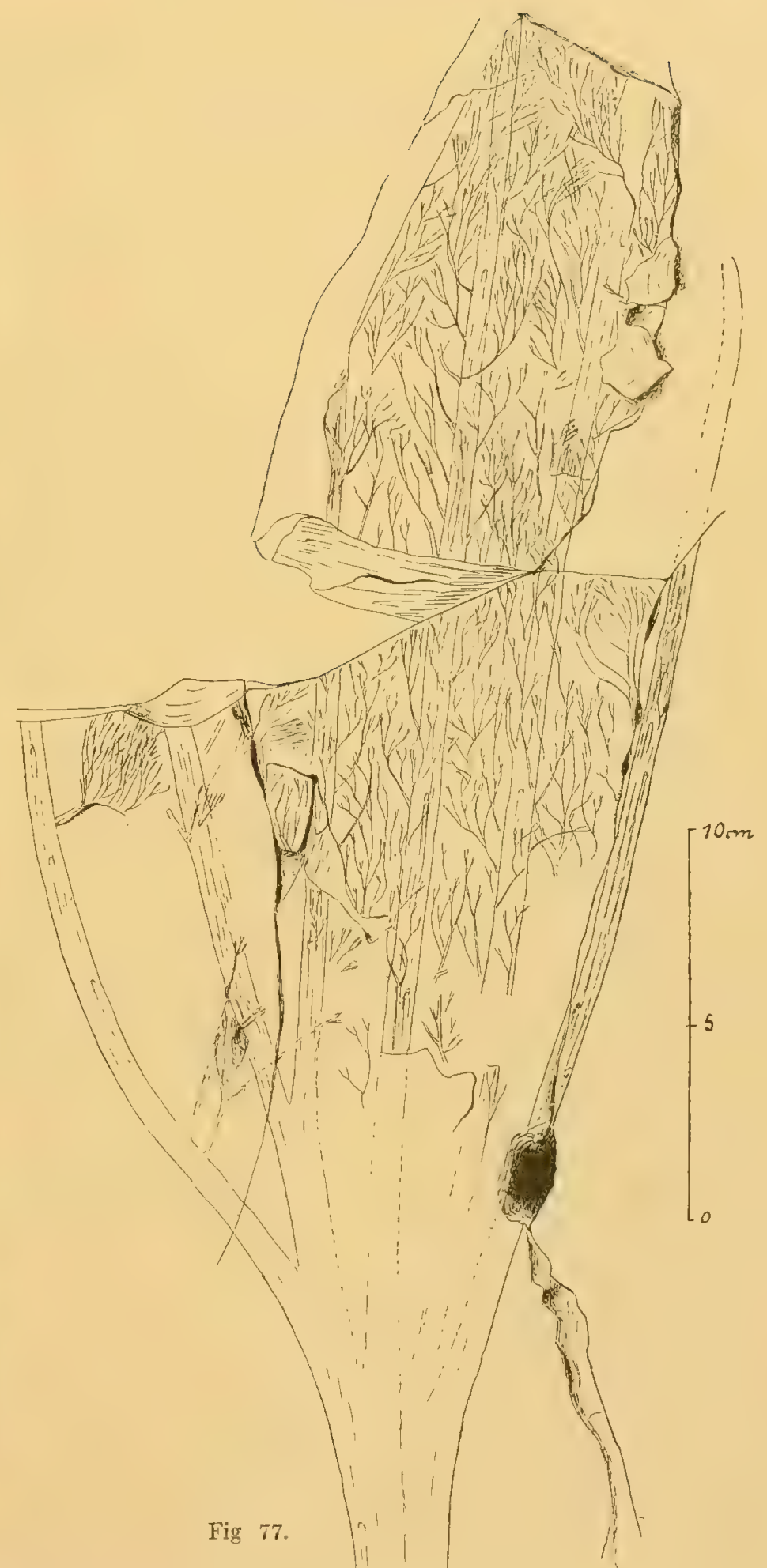

Fig. 76-77. Pseudosporochnus Krejěii, (Stur ex parte) P. et B.

76. Dichotomie. Infiltrations?

77. Srbsko. (Hofmuseum, Wien.) Dichotomies, ramifications ultimes rentlées $\frac{1}{n}$ 
sont rendues peu claires par suite des infiltrations minérales (fer) qui élargissent leurs rameaux (fig. $74,75,78,80$ ).

Sur le tronc d'un de nos fossiles (fig. 70), nous avons rencontré des fragments de houille que nous avons fait macérer et nous avons été assez heureux pour obtenir des fragments d'hỵdrostéréides

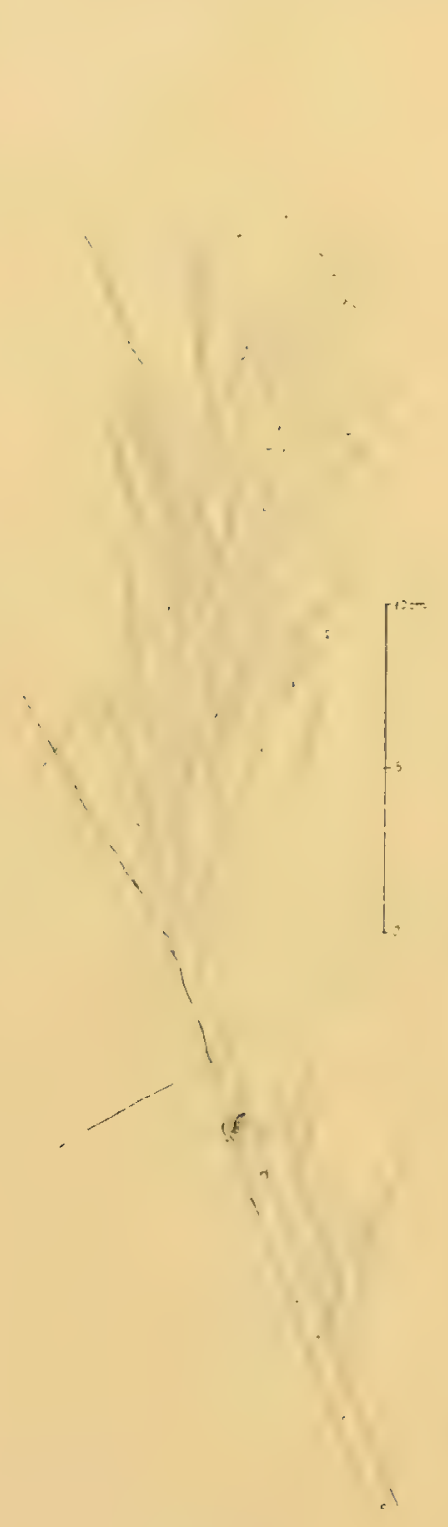

Fig. 78 .

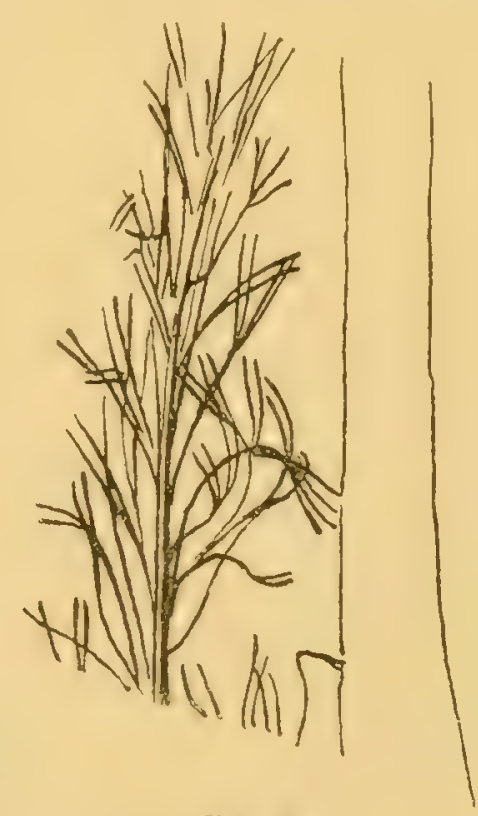

Fie so.

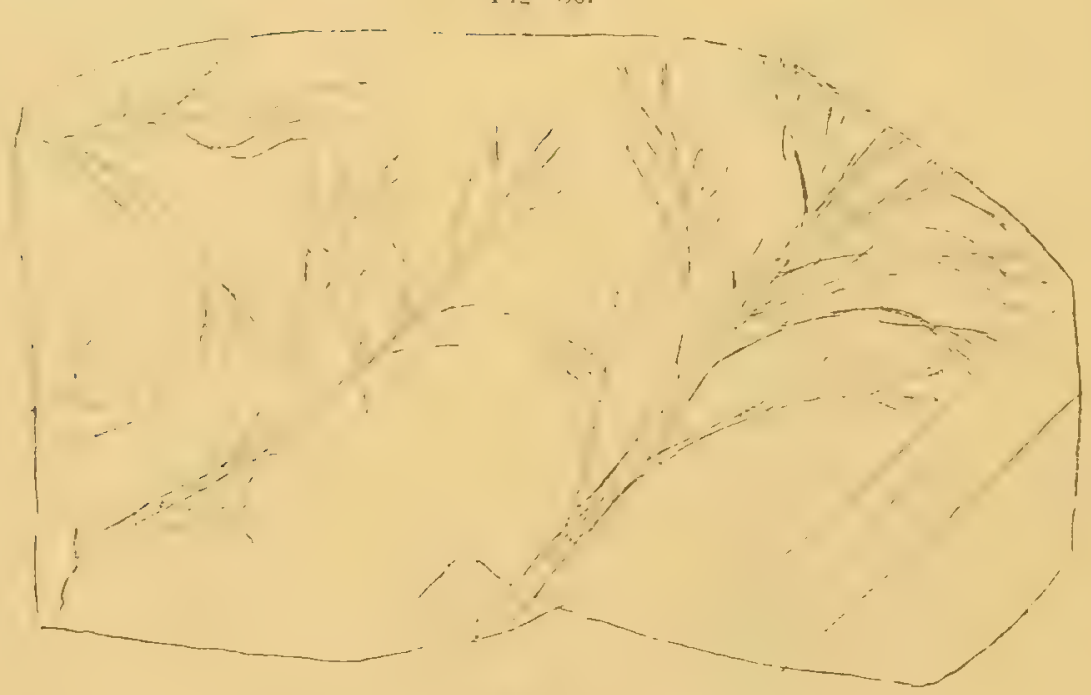

Fin. 79

Fig. 78-80. Pseudosporochnus lírejǔii, (Stur ex parte) P. et B.

78. Srbsko. (Hofmuseum, Wien.) Ramifications cassées. Infiltrations? $\frac{1}{3}$.

79. I (Böhm. Univ. Prag.) Original de Stur, Pl. II, fig. 2. Extrẻmités renflées ’

80. $n(n, n)$ Original de Stur, PI. II, fig. 3. Infiltrations minérales entourant les rameaux $\cdots$

(fig. 70 b) avec des ponctuations aréolées très nettement constituées et disposées en une série seulement. Preuve que ces plantes possédaient du bois. Nous avons pu constater aussi des éléments variés plus ou inoins annelés ou réticulés, et des passages de ces éléments entre eux et aux vaisseaux scalariformes (fig. $70 \mathrm{~b}$ ). 
Le Geologisches Landes-Museum de Berlin possède des échantillons venus du Dévonien (Lenneschiefer) de Westphalie (Gräfrath) et qui montrent des restes très analogues au Psendosporochnus par la ramification au sommet du tronc et par les marques knorrioïdes. Irais nous n'avons pas pu reconnaître sur ces empreintes, toujours brisées près de la ramification dichotomique, si la base était élargie de façon caractéristique comme dans nos échantillons.

Nous nous sommes naturellement demandé à quel groupe de plantes on pouvait rattacher ce reste végétal. Nous avons de primeabord écarté l'idée des Algues, car les Knorria d'une part, les trachéides de l'autre, parlent contre cette interprétation. La présence de trachéides qui rappellent celles des Conifères pouvait faire penser aux Gymnospermes. Mais les trachéides seules ne pourraient pas être une raison pour caractériser une plante comme Gymnosperme, puisqu'on trouve des ponctuations aréolées, disposées, il est vrai, sur plusieurs rangs longitudinaux chez les Calamariées et parmi les Cycadofilices (notamment chez les Lyginoptéridées) etc. (Voir Potonié dans Engler et Prantl, loc. cit.)*)

La présence de renflements aux extrémités ultimes des ramifications pouvait éveiller un rapport avec les Hyménophyllacées, car on aurait pu interpréter ces renflements comme des sporanges. Mais les autres caractères ne concordent pas. Nous devons signaler aussi le fait que Nathorst a décrit sous le nom de Codonophyton epiphyticum du Déronien de l'Ile des Ours des restes qui rappellent de très près les ramifications terminales de nos types de Bohême, et lui-même a saisi ce rapport quand il dit (p. 47): "Siur a décrit quelques restes de l'étage $\mathbf{H}-\mathbf{h} \mathbf{1}$ du Silurien de „Bohême (qui est maintenant admis comme Dévonien) sous le nom "de Sporochnus Krejčí, et ces restes pourraient être comparés "dans une certaine mesure arec Codonophyton." Mais Nathorst, qui a classé ces débris dans les "Incertre sedis", ne sait pas comment les interpréter.

Mac' Coy, dans son Prodromus, a décrit, en 1876, des restes tout à fait indistincts sous le nom de Cordaites australis. Ces restes du Dévonien supérieur ont de l'analogie avec les nôtres.

Quant à nous, nous avons été frappés par l'apparence extérieure de ces plantes et le rapport qu'elle offre avec celle des Psilotacées. Si nous examinons un Psilotum triquetrum, p. ex., nous voyons les tiges se ramifier dichotomiquement et pouvoir présenter souvent, par la rapidité avec laquelle s'est faite une seconde dichotomie, une apparence trichotome, ce qui rappelle très exactement les formes fossiles que nous avons décrites. De plus, sur la tige des Psilotum, il y a de petites feuilles très espacées; nos bourrelets knorrioïdes pourraient être l'indication qu'il y avait aussi dans nos fossiles de petites feuilles sur le tronc. Enfin si nous consultons le travail de Bertrand sur les Tmésiptéridées, nous trouvons, p. 292, que le bois de ces plantes possède des ponctuations

*) Voir aussi à ce sujet, plus loin, p. 58.

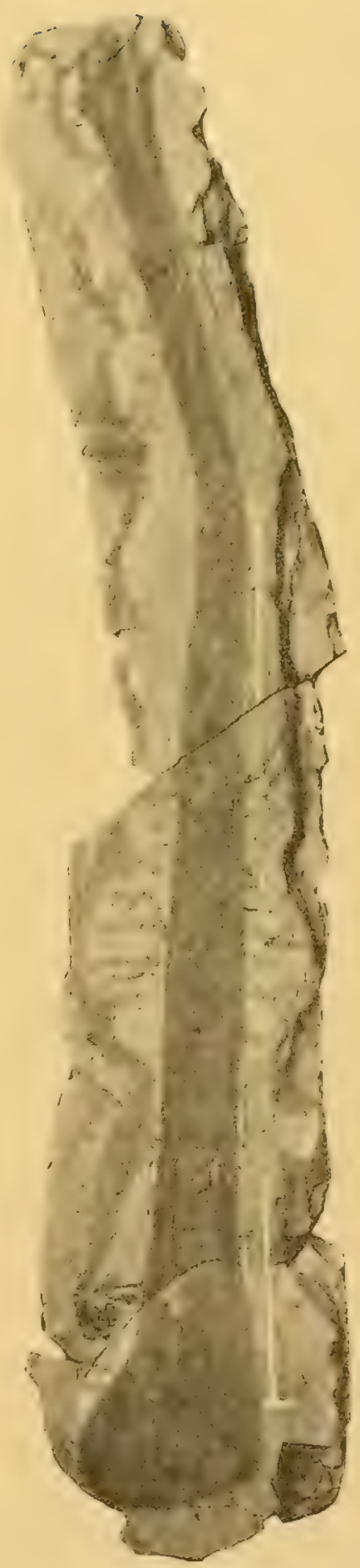

Fig. 81. Pseudosporochnus Krejèiii, (Stur ex parte) P : et B.

81. Srb̌sko, (Geol. Landesmuseum, Berlin.) Photographie d'un grand échantillon montrant la base élargie, comme renflée en bulbe Environ $\frac{1}{10}$. 
elliptiques en une série et pouvant être bordées d'une aréole étroite, ce qui concorderait tout à fait arec ce que nous avons obtenu par la macération de notre houille. Il est rai que nos ponctuations sont beaucoup plus grandes, plus rondes que celles rencontrées chez les Psilotum; mais ce n"est pas un argument contre notre interprétation, car on voit l'anatomie des plantes fossiles présenter souvent des caractères très différents de celle des plantes actuelles correspondantes. Il n'y aurait que la question des très fines ramifications terminées par un renflement (sporange?), qui pourrait s'opposer à la présence de ces fossiles parmi les Psilotacées et parler plutôt en fareur des Fougères. C'est ainsi que ces renflements terminaux pourraient faire penser aux Calymmotheca des Houilles (Lehr)uch de Potonié, p. 103) et aux Dimepteris du Dévonien (Schmalhausen, 1594, p. 30). Mais d'une part, certains auteurs ont considéré comme terminaux les sporanges des Tinésiptéridces (Psilotum et Tmesipteris); d'autre part, il se pourrait que nous fussions ici en présence d'un type mixte, analogue au type mixte des Cycadofitices et qui présenterait des caractères de Fougères et de Psilotacíes. L'état de nos connaissances ne nous permet pas de résoudre définitivement cette question.

Nous avons voulu seulement relever ce point jmportant: Il serait intéressant que la plante que nous arons décrite fût une Psilotacée, qui serait aux petites Psilotacées actuelles un type géant, ligneux, presque arborescent. Ce serait le type prédit, attendu et cherché par tous les paléobotanistes qui se sont occupés des flores siluriennes et déroniennes, et qui voulaient trourer cet ancêtre des Psilotacées actuelles. Il est même curieux que tous cenx qui se sont nccupés de ces fossiles de Bohême n'aient pas aperçu plus tôt ce rapport. Notre Pscudosporochnus serait à peu près aux $P$ silotacées ce que les Lépidophytes sont aux Lycopodiales récentes.

Les Psilotacées sont certainement des plantes en roie de régression, tout le prouve, et surtout leur distribution géographique. Aussi il était à préroir, et beaucoup l'avaient prévu, que l'on découvrirait une ancienne extension de ces plantes curjeuses arec des formes plus ou moins rapprochées des formes actuelles. Bertrand avait annoncé limportance des Tmésiptéricées à ce point de rue et il dit (p. 2ら6): „Je pense aussi qu'il peut être utile aux paléo-botanistes de connaître mieux „quills ne penrent le faire aujourd'hui l'organisation des types anciens que l'on compare aux Lepi"iodendron et aux Psilophyton. Cette application de règles toutes nouvelles montrera en inême temps „à ces sarants les points qu'ils devraient s'efforcer de mettre en relief lorsqu'ils ont à indiquer les "affinités probables des genres fossiles disparus aujourd'hui." (Voir aussi it ce sujet Solms.)

\section{Incertie sedis.}

Nous derons décrire ici un certain nombre de débris que nous ne sarons comment interpréter, qui sont trop peu clairs ou trop petits pour se laisser rattacher à une espèce ou à une autre, ou qui sont pourtus d'organes dont nous ignorons la signification. Ils sont généralement trop mal conservés pour que nous puissions leur attribuer un nom spécial; cependant certains caractères nous autorisent à les placer ici plutôt qu'ailleurs.

1", fig. 82. Nous avons pu constater une sorte de dépression elliptique entourée d'un bourrelet sur un fragment de Pseudosporochnus Krejěii, dont nous possédions aussi la contre-empreinte et qui, ramifié de façon très typique, portait des Knorvia très caractérisées. Nous ne savons à quoi rapporter le détail en question; est-ce un organe de la plante? Est-ce un petit organisme qui se serait incrusté là? Nous lignorons, mais cette marque est si nette qu’elle était intéressante à sjgnaler.

$2^{0}$, fig. S3. Un axe ramifé dichotomiquement; c'est pour cette raison que nous l'arons placé ici; mais l'axe principal et ses ramifications portaient des cicatrices curieuses, plus ou moins elliptiques, déprimées, arec une marque centrale (trace fasciculaire?). Ces cicatrices étaient raguement disposées en spirale. 


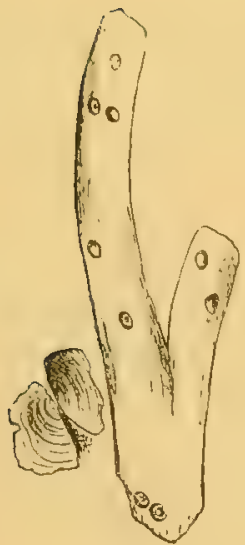

Fig. 83 .

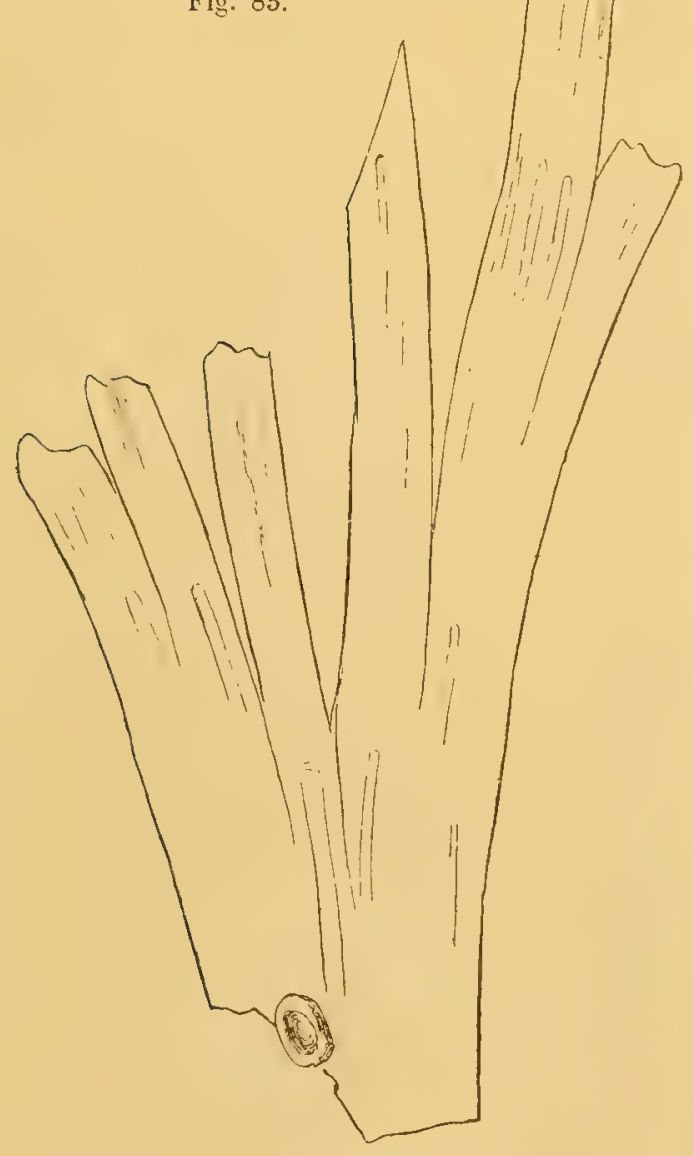

Fig. 82.

Fig. 82-85. Incerta sedis.

82. Srbsko. (Geol. Landesmuseum, Berlin.) Pseudosporochnus Krejčí ramifié en éventajl. Tinorria. A la base de l'échantillon un bourrelet circulaire $\frac{1}{1}$.

83. Srbsko. (Geol. Landesmuseum, Berlin.) Axe dichotome; cicatrices. A gauche l'empreinte $d^{\prime}$ une coquille $\stackrel{1}{1}$.

84, 85. Hostim. (K. k. geol. Reichsanstalt.) Axe ramifié. Knorria $\frac{1}{1}$.

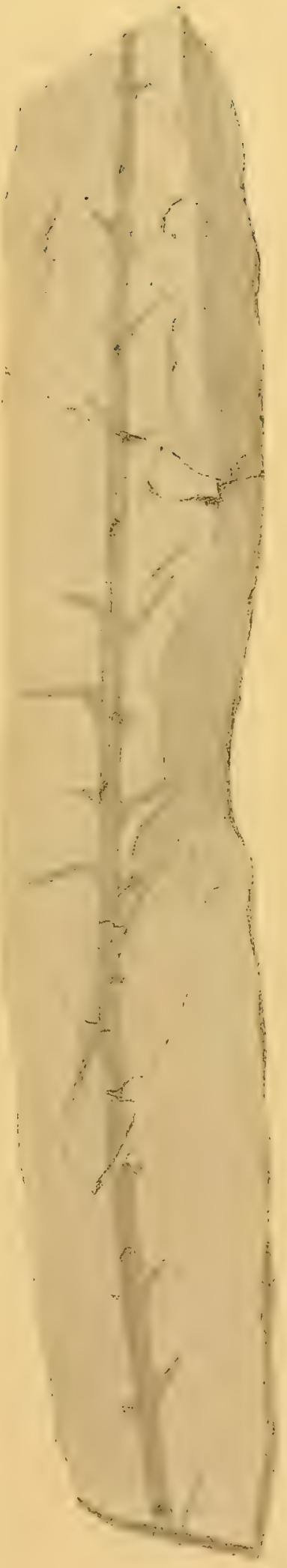

Fig. 81 .

l'ig. \&5.

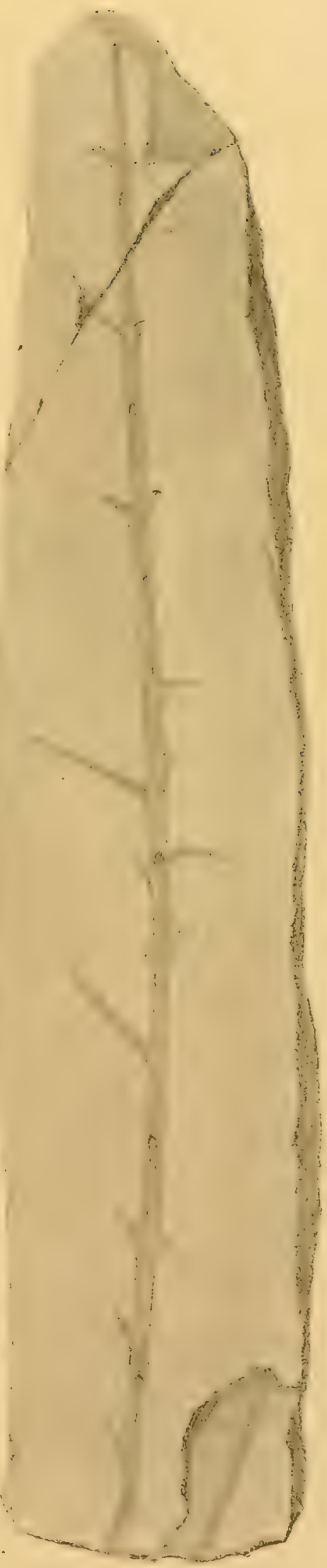


30. fig. 84-S6. Des branches ramifiées latéralement et possédant des Knorria plus ou moins indistinctes; elles pourraient se rapporter aux branches de Pseudosporochnus, dont les ramifications ultimes auraient été cassées. ( $C f$. fig. 70.)

$4^{\prime \prime}$, fig. 87, 88. Axes ramifiés. Ce sont peut-être des ramifications de troncs de Pseudosporochmus; il semble, dans la fig. 87 , que l'on voie un axe central.

50, fig. 89, 90. Axes ramifiés, dont les terminaisons sont pourvues de parties rentlées qui pourraient correspondre peut-être aux renflements cités chez Pseudosporochnus. Il se peut aussi que ces renflements, très gros ici, et à contour assez indistinct, aient été exagérés par des infiltrations minérales (fer?).

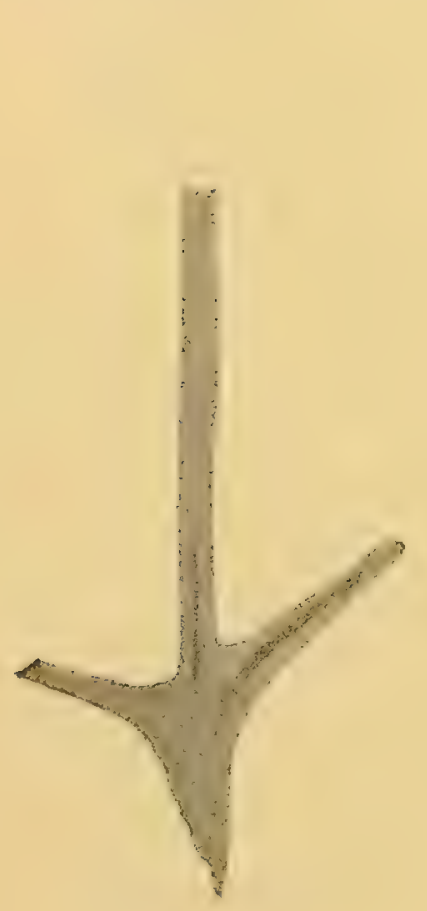

Fig. 83.

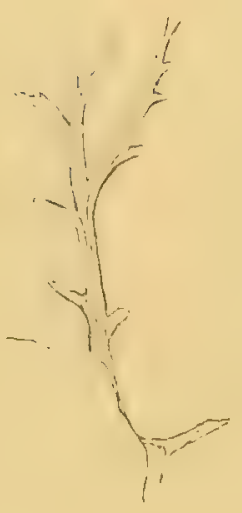

Fig. 86 .

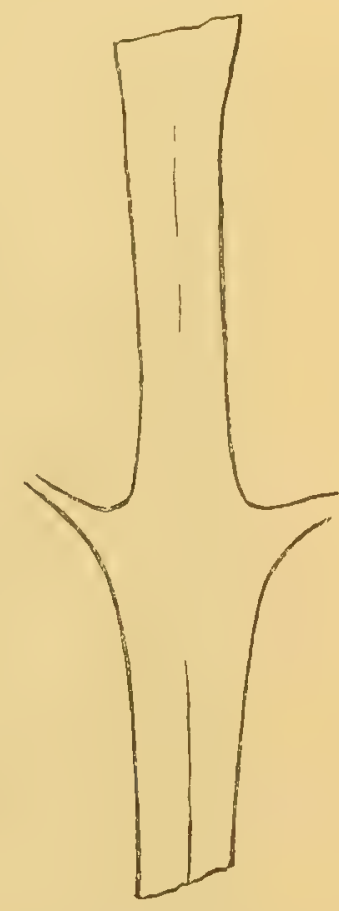

Fig. 87.

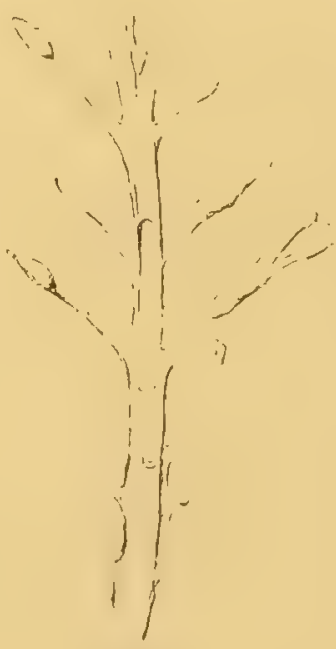

Fir. 90.

Fig. 86-90. Incerta sedis

86. Srbsko (Böhm. Univ. Prag.) Axe ramifié ! 1 .

87. " (Reichsanstalt, Tieu.) Axe ramifié -1.

88. Ramifications dichotomiques. Axe central ?

89. Hostim. (K. k. geol. Reichsaustalt.) Formations renflées (concrétionnaires?). Env. I

90.

Protolepidodendron harlsceini, P. et B. et Protolepidodendron Sehreryaneme, Kr.

Fig. $91--102$.

Chauvinia Scharyana, Stur.

Dicranophyllum austraticum, Dawson.

? Bothrodendron (Cyclostigma) brevifolium, Nath.

Lepidodendron Veltheimianum (?) Ettingsh., (in Stur).

Nous avous donné deux uoms spécifiques aux restes que nous rangeons provisoirement dans le genre Protolepidodendron. 
1. Protolepidodendron karlsteini, P. et B.

11 s'agit tout d'abord de branches assez étroites (fig. 91-93) trouvées à Karlstein et à Srbsko en Bohême; ces branches sont couvertes de feuilles petites, mais assez larges, ovoïdes-lancéolées, et nous les

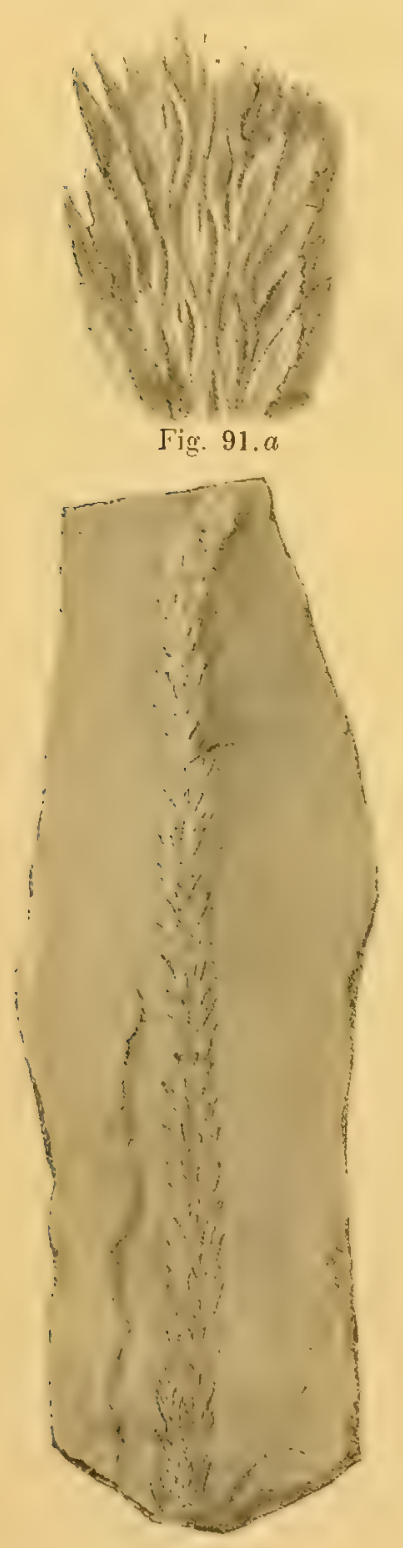

Fig 91.6
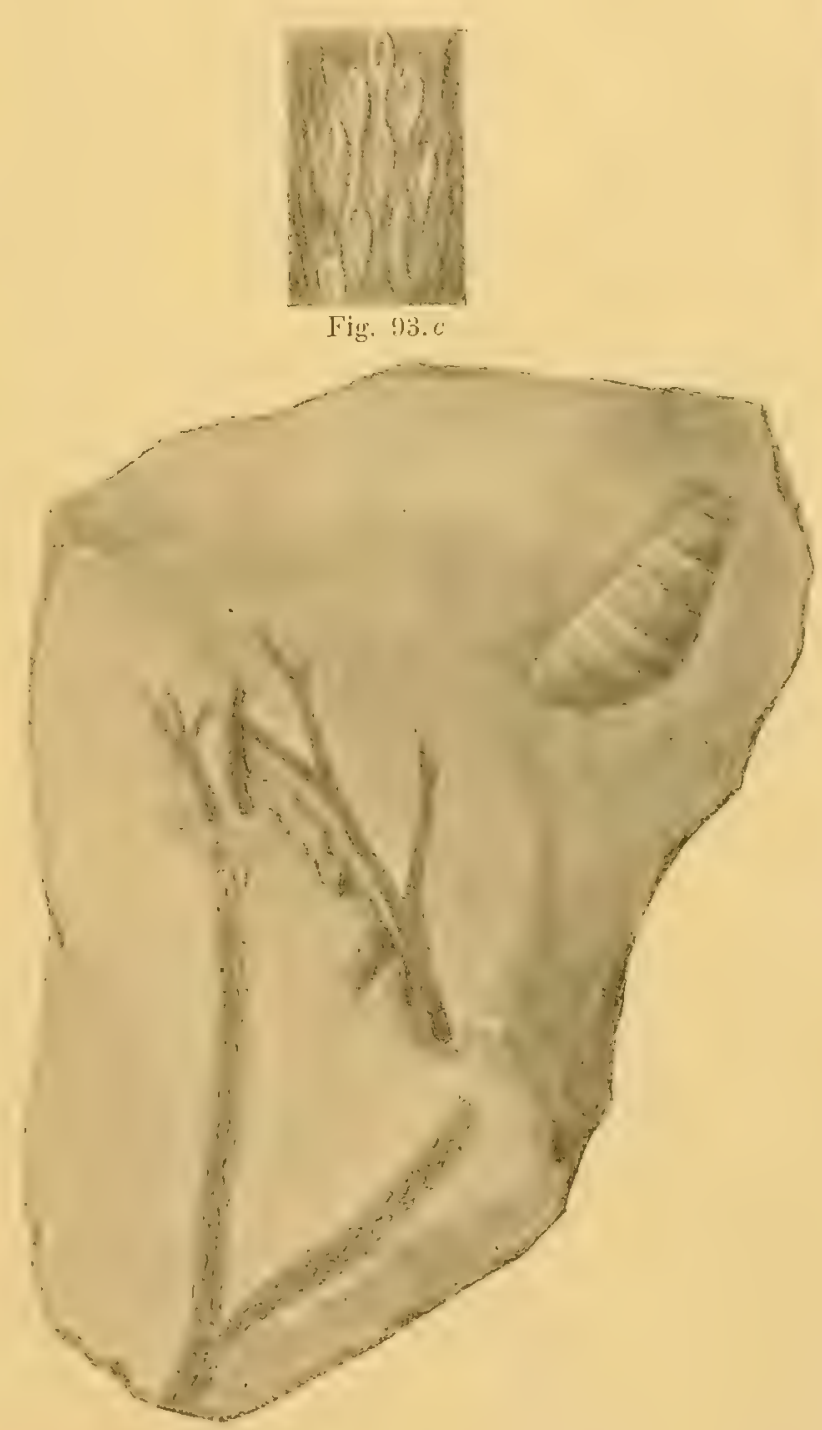

Fig, 93.a

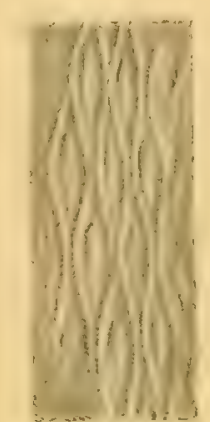

Fig. 93.6

Fig. 91-93. Protolepidodendron karlsteini, P. et B.

91. Karlstein. (K. k. geol. Reichsanstalt.) Axe portant de petites feuilles assez larges. Coussinets lépidodendroïdes. $a \cdot{ }^{5}, b \quad \frac{1}{1}$. 92. Srb̋sko. (Collection Dusl.) Axe portant de petites feuilles lancéolées $\frac{1}{1}$.

93. $" \quad$ (K. k. geol. Reichsanstalt.) Feuilles, élargies, coussinets lépidodendroüdes, empreinte d'animal. $a \frac{1}{1}, b$ et $c \frac{5}{1}$.

avons représentées (fig. $91 a, 93 b, c$ ), agrandies 5 fois, de façon qu'on puisse y reconnaître les détails importants. Ces branchettes rappellent assez exactement l'apparence de certaines Lépidodendrées, d'autant plus qu'en certaines places, où les feuilles ont été enlevées, on peut voir les coussinets 
foliaires, vraisemblablement bergérioïdes, c'est-ì-dire dépourvus de leurs tissus périphériques et très nettement caractérisés, arec une trace de faisceau au sommet du coussinet (fig. 91a).

\section{Protolepidodendron Scharyanum, Kr.}

Les autres lébris, trouvés ì Srbsko, se présentaient (fig. 94-102) comme branches très semblables aux précédentes, étroites, de $\frac{1}{2}-1^{\mathrm{cm}}$ de large, dichotomes, couvertes de coussinets plus ou moins rhomboïdaux (fig. $96 b, 98 b, c$ ) que l'on pourrait aussi prendre, arec leur trace fasciculaire plus ou moins centrale (fig. 96 b) pour des états Bergeria. Il se pourrait cependant, les marques étant centrales, que ces cicatrices foliaires fussent analogues à celles qui caractérisent généralement les coussinets complets des Bothrodendracées. Ces coussinets peuvent être assez rariables d'apparence comme en témoignent nos figures, agrandies ou non.

Mais ces débris ne sont pas, comme les précédents, couverts de feuilles plus ou moins larges; on remarque au contraire, sur les bords, des empreintes assez rares, très déliées, presque filiformes (fig. 956 ) et parfois partagées au sommet, de façon tout à fait inattendue, par une petite dichotomie (fig. $98 a, l$ ). Quelquefois aussi, on voit ces appendices s'attacher au bord de la tige par une base un peu élargie, et s'allonger en s'amincissant, sans se diviser (fig. $95 a$ et $b$ ).

Dans les fouilles que nous avous fait faire, nous arons trouvé (fig. 99) un échantillon très élégant, montrant un axe, et, sur les côtés, des feuilles très finement terminées en deux pointes. Ce type peut se rapporter à ceux décrits ci-dessus, quand même nous n'y trouvons pas les cicatrices caractéristiques de la surface. Nous voyons, en effet, un axe central fort bien conservé, et des deus côtés de cet axe il y a une zone brune, à l'extérieur de laquelle sont les feuilles bifurquées. Cette zone correspondrait aux tissus extérieurs à l'axe central.

Nous avons laissé ces types 1 et 2 réunis sous un même nom de genre, car les cicatrices, quoique présentant entre elles des dissemblances assez notables, nous ont permis ce rapprochement. Il se pourait cependant qu'il fût préférable de placer le type à feuilles larges parmi les Lycopodites ou d'autres Lépidoploytes.

Stur a fait de ces fossiles une Siphonée: Chauvinia Scharyana, (Kr.) Stur. Ici encore nous ne comprenons pas que Stur ait pu décrire comme Algues les types qu'il dessine et qui possèdent des coussinets lépidodendroïdes aussi nettement caractérisés. Stur indique, dans sa PI. I, fig. 1, les feuilles filiformes toutes dichotomes au sommet. Il dit (p. 334) „qu'un échantillon de Chauvinia ${ }_{n}$ Scharyana fut communiqué par Barrande à Ettingshausen et que ce dernier le tenait pour un "Sagenaria (peut-être Lepidodendron Veltheimianum). Mais on acquiert le mieux, ajoute Stur, Ja "conviction que ce reste ne peut appartenir à un Lepidodendron, si l'on compare ma figure avec "les branches de même largeur des vrais Lepidodendron par exemple de $L$. Veltheimianum." Il est intéressant pour nous de constater que les coussinets de ce type rappellent ceux des Lepidodendron et que v. Ettingshausen l'avait déjả remarqué. Mais les échantillons que nous possédons sont trop incomplets pour qu'on puisse les rattacher au genre Lepidodendron si bien caractérisé. Un autre point doit nous faire repousser immédiatement toute idée d'Algue, c'est que, par une macération convenable de la houille que nous avous rencontrée dans la pièce dont nous parlions plus haut (fig. 99), nous avons pu trouver des éléments vasculaires, entre autres de très jolies trachéides scalariformes (fig. 99 b).

Krejči avait décrit ces débris comme Protolepidodendron Scharyanum, Kr.

Ils pourraient se rattacher peut-être anx Bothrodendracées qui sont les Lépidoplıytes généralement répandus dans les terrains dévoniens. On sait que les Bothrodendron sont remarquables par le fait que les coussinets lépidodendroïdes qu'ils ont sur les branches jeunes, s'effacent rapidement et que les rameaux passent en vieillissant à l'état léioderme. Nous n'avons pas pu trouver de tels 
états léiodermes, mais il faut dire qu'aucun de nos échantillons ne dépassait $1 \frac{1}{2} \mathrm{~cm}$ de largeur. Aussi ne serait-il pas impossible que nous fussions en présence de branches jeunes de Bothrodendracées, sans que nous priissions l'affirmer à coup sûr.

La présence de feuilles (?) dichotomes au sommet, chez une de nos espèces, ne parlerait pas contre l'interprétation de ces fossiles comme Bothrodendracées, quoique celles-ci n'aient pas de feuilles divisées. Il serait même intéressant de constater chez une Bothrodendracée très ancienne la présence de feuilles dichotomes, ce qui serait l'équivalent de ce qu'ou retrouve chez les Equisetales, dont les plus anciennes (Protocalamariacées) ont des feuilles bifurquées qui ont disparu chez les fossiles plus récents *).

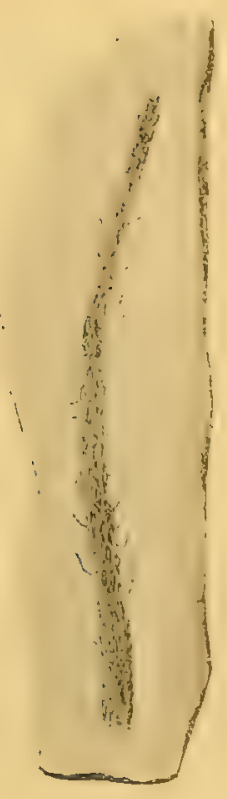

Fig. 95. a

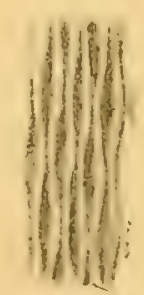

Fig. $96 . b$

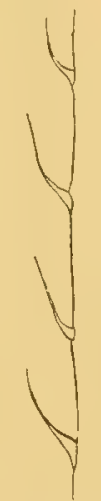

Fig. 95.b

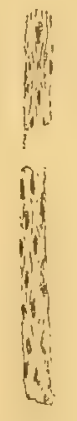

Fig. 91.

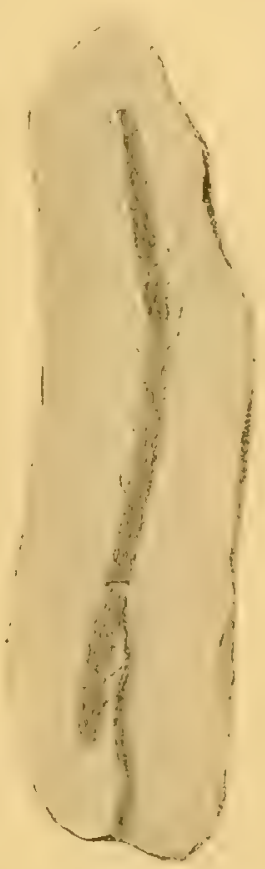

Fig. 96. $a$

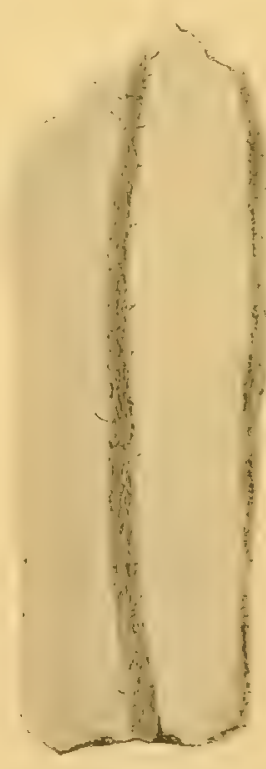

Fig. 97.

Fig. 94-97. Protolepidodendron Scharyanum, P. et B.

94. Srbsko. (Reichsanstalt, Wien.) Coussinets lépidodendroïdes $\frac{1}{1}$.

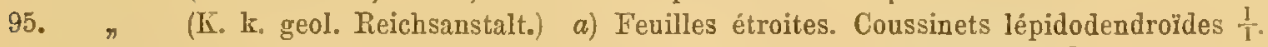

b) montre la base large des feuilles $\frac{5}{1}$.

96. Srbstio. (K. K. geol. Reichsanstalt.) Feuilles étroites, coussinets lépidodendroildes. $a \frac{1}{1}, b \frac{5}{1}$.

97. $"(n, " n), " n, \frac{1}{1}$.

Nous devons citer ici 3 figures de M. Vlček, dont M. Fritsch nous a communiqué les dessins; nous avons pu voir qu'il avait, comme nous, remarqué sur les restes à coussinets lépidodendroïdes des feuilles curieuses, élargies à la base, puis très étroites et bifurquées au sommet. Nous reproduisons (fig. 100-102) trois de ses dessins qai montrent bien de quoi il s'agit.

Des observations semblables ont été faites par Dawson qui a décrit, du Dévonien d'Australie, des fossiles absolument identiques à ceux qui nous occupent, avec les mêmes cicatrices le long du tronc, et les mêmes feuilles bifurquées au sommet. Il les a appelés Dicranophyllum australicum. Il ajoute que le nom Dicranophyllum a été établi par Grand' Eury pour certaines plantes des schistes houillers français, et que cet auteur regardait ces plantes comme probablement des Conifères.

*) Voir p. 24. 
Nathorst, comme Heer avant lui, dans son "Ile des Ours" a décrit beaucoup de restes de Bothrodendracées qui concordent bien au point de vue des rameaux et des coussinets foliaires

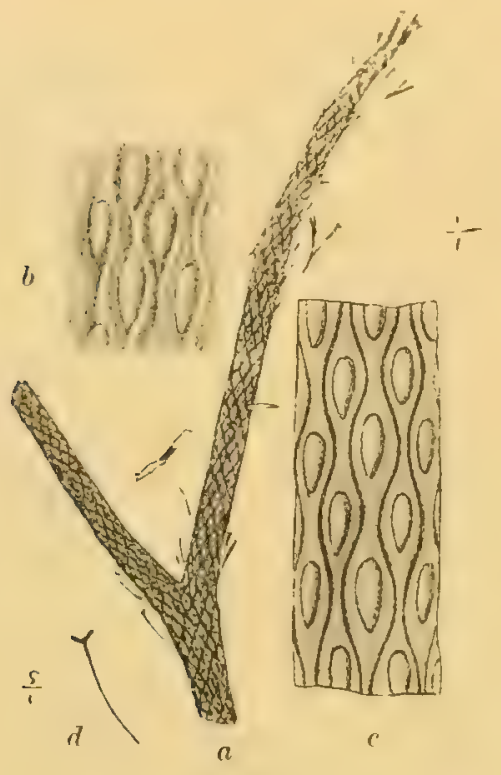

Fier. 98 .

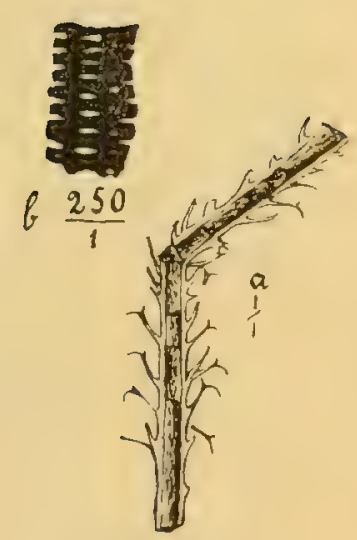

Fig. 99.

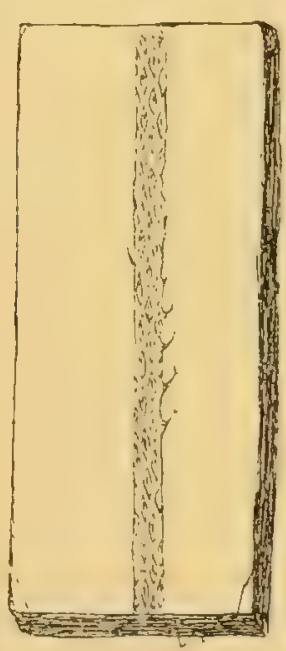

Fig. 101.

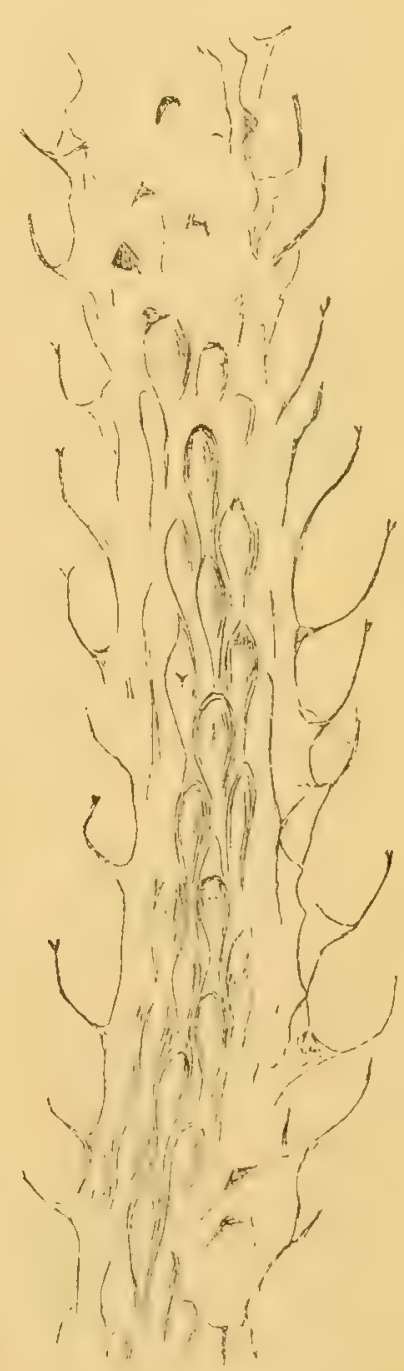

Fig. 100 .

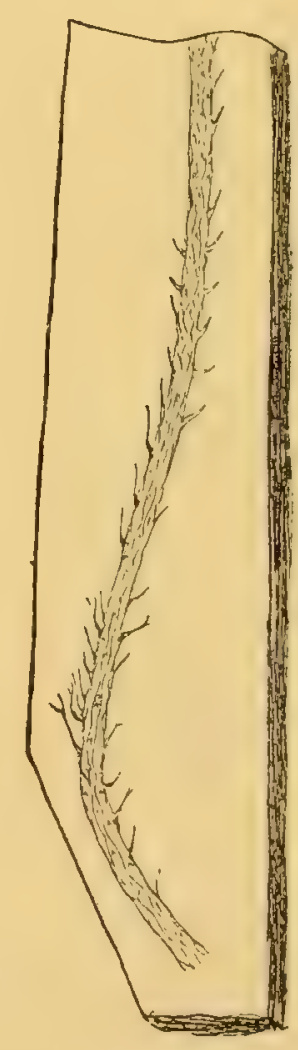

Fig. 102.

Fig. 98-102. Protolepidodendrou Scharyanum, P, et B.

98. Srbsko. (K. k. geol. Reichsanstalt.) a Coussinets lépidodendroïdes $\frac{1}{1}, b, c$ id. 1 , $a$ feuilles bifurqquées au sommet $\frac{3}{1}$.

99. Srbsko. (Geol. Landesmuseum, Berlin.) $a$. Axe central rempli de houille. Petites feuilles élargies a la base, bifurquées au sommet $\frac{b}{1}$ b. Une hydrostéréide obtenue par macération de la houille 250

100. Dessin de M. Vlček. Coussinets lépidodendroldes, feuilles bifurquées an sommet (plusieurs fois grossi). $101-102$.

avec nos types de Bohême. La plupart de ses échantillons, qui sont heancoup plus gros que les nôtres et ont nettement les caractères de Bothrodendron avec les formes léiodermes typiques, n'ont malheureusement que rarement des feuilles. Un de ses dessins cependant inontre, comme les nôtres, 
des feuilles filiformes, et parfois, semble-t-il, dichotomiques au sommet. C'est ce que Nathorst appelle Bothrodendron (Cyclostigma) brevifolizm, Nath.") Potoniẻ a cité aussi (Silur-Flora) un Bothrodendron pour des schistes siluriens du Harz. Done l'hypothèse d'une Bothrodendracée ne peut pas être repoussée a priori. Quant au nom à attribuer ì ces types, nous pourrions ieur donner celui de Dicranophyllum, qui semble le mieux indiquer leur identité et qui aurait l'avantage de relever cette curieuse coïncidence de la présence de types identiques, à la fois en Bohême et en Australie. Mais ce nom aurait l'inconvénient, selon la conception de Grand' Eury, de rappeler une parenté (peu probable) avec les Conifères. Nos fossiles pourraient aussi être rangés dans le genre Bothrodendron; cependant, puisque aucune raison fondamentale ne s'oppose au nom donné par Krejăi, nom qui a pour lui l'avantage de l'ancienneté (1879), nous conserverons à nos fossiles le nom de Protolepidodendron Scharyanum, Kr. pour les échantillons à feuilles dichotomiques, et nous appelerons $F$. Karlsteini le type à feuilles plus larges. Des recherches ultérieures pourront seules, par la découverte d'échantillons meilleurs, permettre d'affirmer, si ces deux espèces doivent être définitivement séparées et dans quel groupe il faut les placer.

\section{Incertae sedis}

Nous devons décrire ici un type incertain (fig. 103) qui se rattache peut-être à nos Bothrodendracées ou qui pourrait être considéré comme une Lépidodendracée. Il s'agit d'un axe portant de nombreuses marques disposées en séries longitudinales très régulières et qu'il est difficile d'interpréter, soit comme Knorria, soit comme coussinets foliaires. Les Bothrodendracées ont souvent, avant d'arriver à l'état léioderme, des apparences qui rappellent celles que nous décrivons, mais d'autre part on a décrit des formes de Lepidodendron très analogues à notre échantillon. Ainsi Dawson, en 186!-1863, puis en 1873, publie un Lepidodendron Gaspianum qui présente les mêmes caractères (marques knorrioïdes) que notre pièce de Srbsko.

Ulodendron (?) hostimense, $P$. et $B$.

Fig. 104.

Les fouilles que nous avons fait faire à Srbsko nous ont fourni un type intéressant qui consiste en un axe assez large, ramifié en dichotomies. Les rameaux sont couverts de stries longitudinales plus ou moins irrégulières et plus ou moins accentuées. Peut-être ne sont-elles qu'accidentelles, mais sur cette impression, dont nous possédons aussi la contre-empreinte, ce qui nous a frappés est la présence, à la base des rameaux, de marques plus ou moins arrondies avec une dépression centrale, et dans cette dépression une espèce de cicatrice. Ces formations rappellent celles décrites sous le nom d'Ulodendron et qui sont constituées par des tiges de Lepidodendron portant les marques d'insertion des cônes cauliflores. Zeiller a rattaché, avec pretuves à l'appui, aux Bothrodendron, sous le nom de $B$. punctatum, un type avec de semblables formations et qui avait été signalé à plusieurs reprises (entre autres par Schimper) sous le nom d'Ulodendron punctatum. Chez notre échantillon, ces marques se trouvant être toujours à la naissance des ramifications, pourraiest être un argument de plus en faveur de la théorie qui veut que les fleurs naissent toujours en un point de moindre accroissement. Ici, chaque rameau de la dichotomie recevant moins de nourriture, il en résulterait un accroissement ralenti, et il est intéressant de constater que c'est en ce point justement que se trouve la fleur. L'analogie est si grande entre les Ulodendron et nos échantillons, que nous avons cru pouvoir adopter pour notre fossile, qui malheureusement est unique, le nom d'Ulodendron (?) hostimense. Mais on ne pourra rien affirmer jusqu'au moment, où l'on aura trouvé ces formations en

*) Mr. Nathorst m'écrit que son $B . b$. n’a pas de feuilles bifurquées. $P$. 

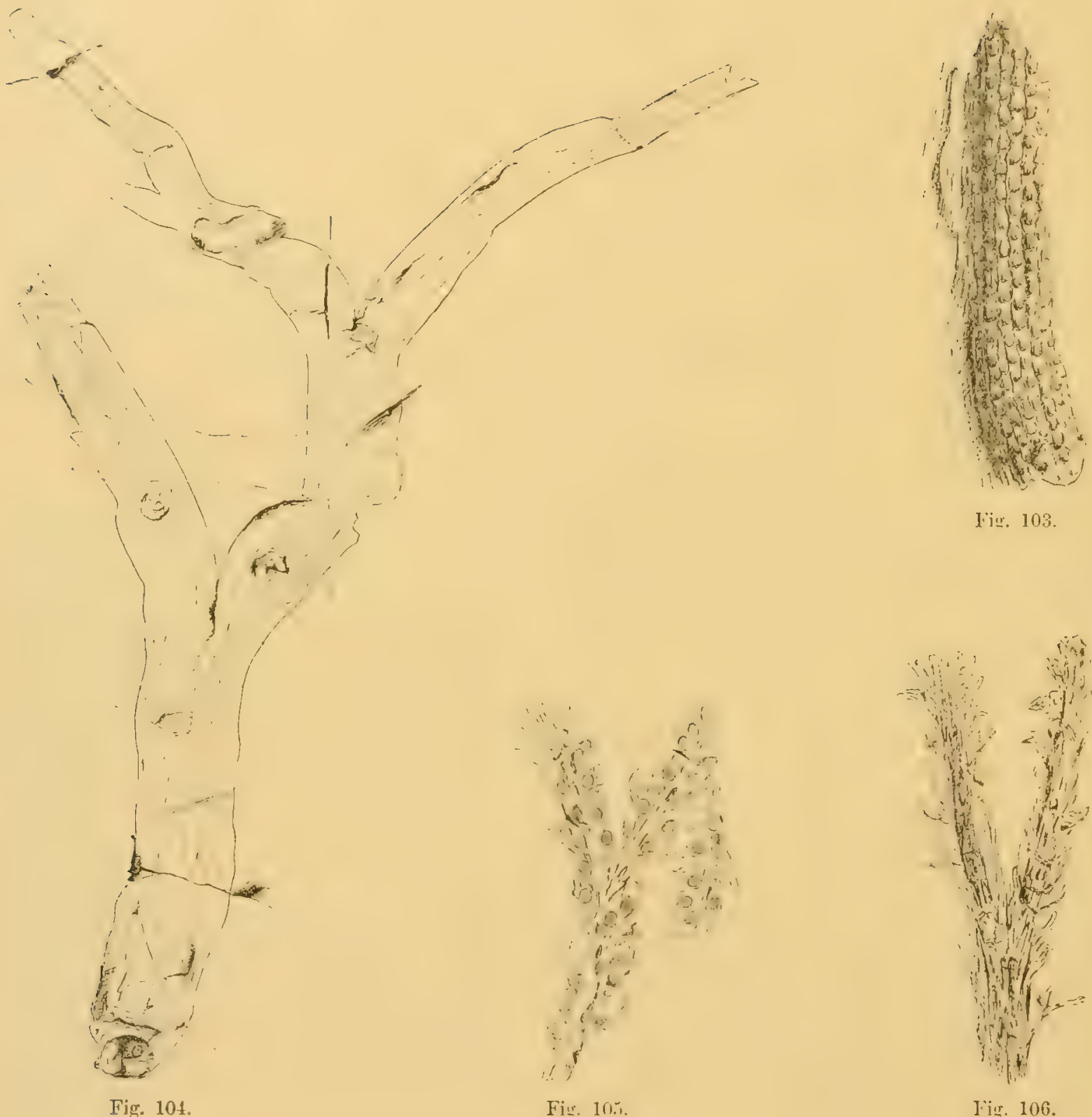

IFị. 103

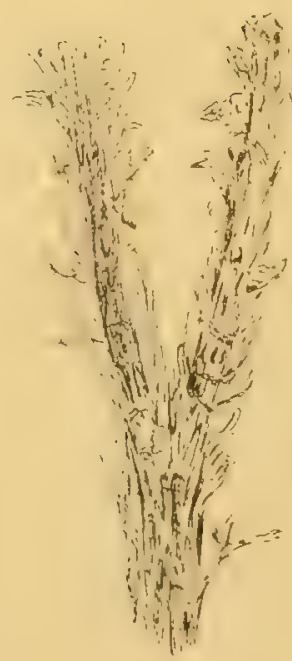

Iiig. 106.

Fig. 103. Incerta selis.

103. Srusko. (Geol. Laudesmuseum, Berlin.) Empreinte lépidodendroïde $\frac{1}{1}$

Fig. 101. Ulodendron(?) hostimense, P. et B.

101. Srbsko. (Geol. Landesmuseum, Berlin.) Tronc dichotomique avec cicatrices ulodendroides $\frac{1}{1 .}$

Fig. 105-106. Iycopodites hostimense, P. et B.

105. Srbsko. (Geol. Laudesmuseum, Berlin.) Axe fertile dichotome; feuilles nombreuses, sporanges $\div$.

106. (Collection Dusl.) Axe feuillé dichotome, stérile.

relation avec des formes typiques de Bothrodendron, et en attendant nous ne pouvons pas prétendre certainement être en présence de vrais Ulodendron. Cependant rien ne vient infirmer décidément 
jusqu'ł présent notre supposition; au contraire puisque nous venons de voir que des restes analogues ì des Bothrodendracées sont fréquents dans l'étage $\mathbf{I I}-\mathbf{h} \mathbf{1}$ de Barrande.

Nous n'affirmons pas que notre Ulodendron appartienne à notre Protolepidodendron; nous nons bornons à les décrire côte à côte, et des recherches ultérieures élucideront peut-être ce point, en mettant au jour des formes typiques de Bothrodendracées avec les cicatrices caractéristiques et portant les marques ulodendroïdes.

Lycopodites hostimensis, $P$. et $B$.

Fig. 105-106.

Nous avons eu entre les mains un fossile dont nous n'avons pas trouvé jusqu'ici l'indication dans les ouvrages traitant du Dévonien de la Bohême. Il s'agit d'une branche feuillée dont nous avions aussi la contre-empreinte; elle était remarquable par la grandeur et le grand nombre de ses feuilles, et par le fait que, sur la plupart de celles-ci, et, semble-t-il, fixés à leur base, se trouvaient des sporanges assez gros $\left(1 \frac{1}{2}-2^{m m}\right.$ de diamètre), circulaires, peut-être un peu elliptiques, mais suffisamment bien conservés pour qu'on puisse reconnaitre leur forme générale. Potonié, dans son Lelıbuch (p. 259) et dans les Ptéridophytes des „Pflanzenfamilien“ d'Engler et Prantl (p. 716), décrit les Lycopodites comme des restes de Lycopodiacées très semblables à des rameaux jeunes de Lípidodendracées.

Il donne quelques exemples qui prouvent qu'il y avait très probablement, dans les terrains paléozoiques déjà, de vraies Lycopodiacées ou des Sélaginellacées, et que certains restes décrits pourraient même être rapportés directement à Lycopodium. On connaît, dit-il, la forme des sporanges, mais on ne peut pas dire, s'ils sont iso-ou hétérosporés. Les feuilles, il est vrai, sont disposées comme chez les Lycopodiacées et les Sélaginellacées, mais il serait imprudent d'utiliser les noms récents de Selaginella ou de Lycopodium. Il est préférable de s'en tenir à l'ancien nom de Goldenberg, qui nommait ces restes Lycopodites. C'est ce que nous avons fait pour l'espèce qui nous occupe, et nous lui avous donné, vu la disposition de ses feuilles, la dichotomie de ses branches, la distribution et la forme de ses sporanges, le nom de Lycopodites (espèce $L$. hostimensis). Il est intéressant de relever ce fait que l'axe fertile (fleur) est dichotomisé, comme cela se rencontre encore chez de nombreuses Lycopodiacées actuelles, notamment chez beaucoup d'espèces tropicales.

Nous avons représenté (fig. 106) un échantillon qui pourrait se rattacher à notre Lycopodites, quoique nous n'ayons pas pu y constater les sporanges; mais la dichotomie de l'axe, les feuilles dont il est couvert, tout parle en faveur d'une similitude.

Han'randeina Duslianer, (Kr.) Stur ex parte.

Fig. $107-122$.

Protolepidodendron Duslianum, Kr. ex parte.

1. Nous avons tout d'abord à considérer des restes végétaux se présentant le plus souvent comme des tiges de $20-30^{\mathrm{cm}}$ de longueur, et dont la largeur varie de $2-4^{\mathrm{cm}}$. Ces branches, comme toujours dans ces dépôts allochtones, sont malheureusement des fragments portés autrefois par des troncs plus considérables, qui ne sont pas parvenus jusqu'à nous.

Ces branches sout caractérisées à leur sommet par une dichotomie remarquable (fig. $107 a$ et $b$, $111,112,115$ ) et chacune des ramifications ainsi produites peut (fig. 111) se dichotomiser à son tour.

Sur les fossiles eux-rnêmes, on remarque des moulages qui peuvent être de deux natures selon les échantillons considérés; sur les uns, ce sont des marques knorrioïdes assez allongées: $20-40^{\mathrm{mm}}$ 


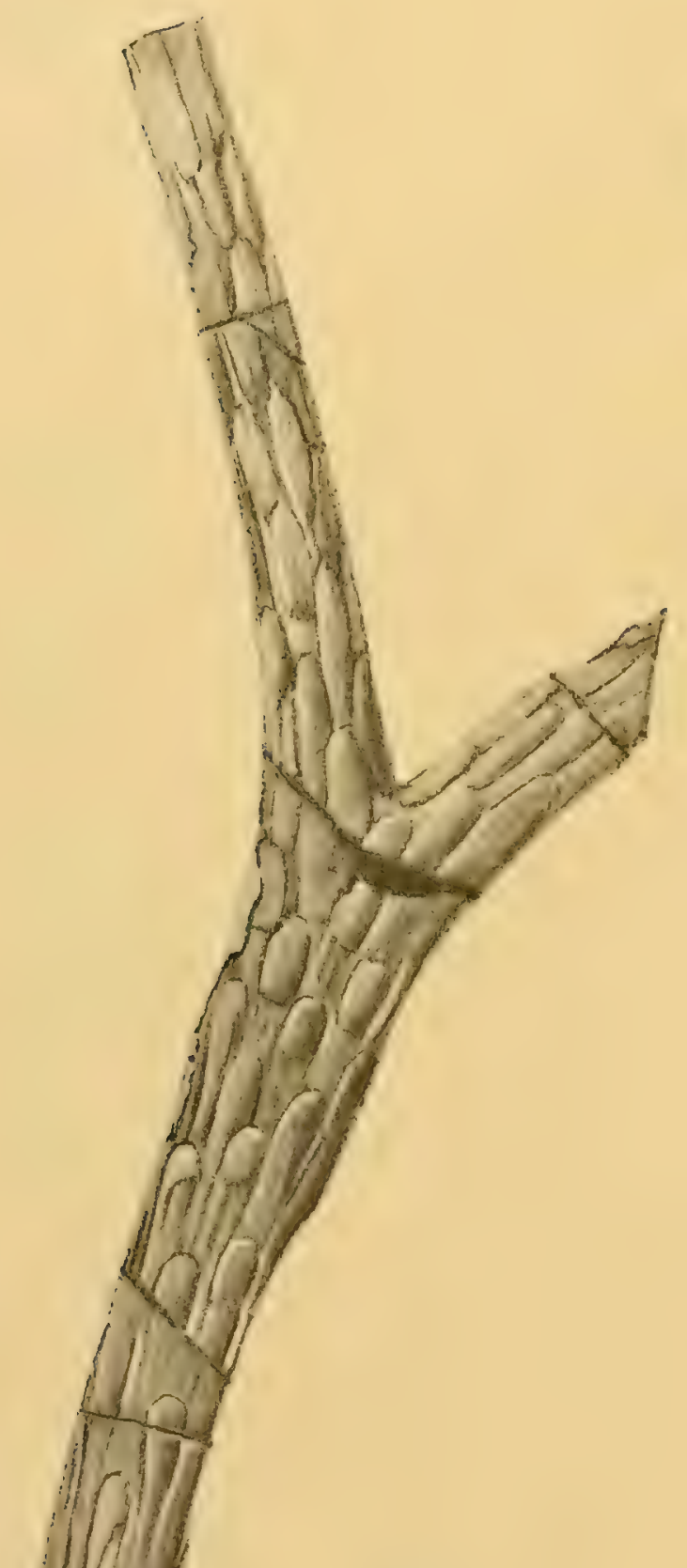

ligr. 117. a

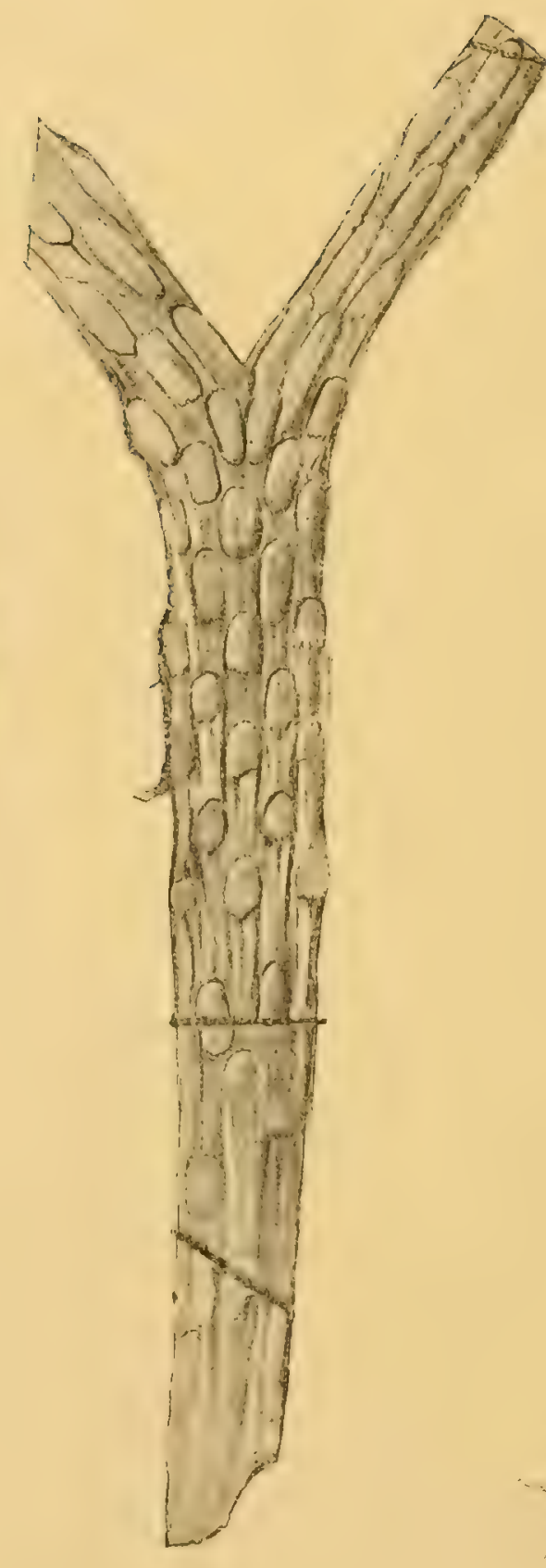

Fig. 107.b

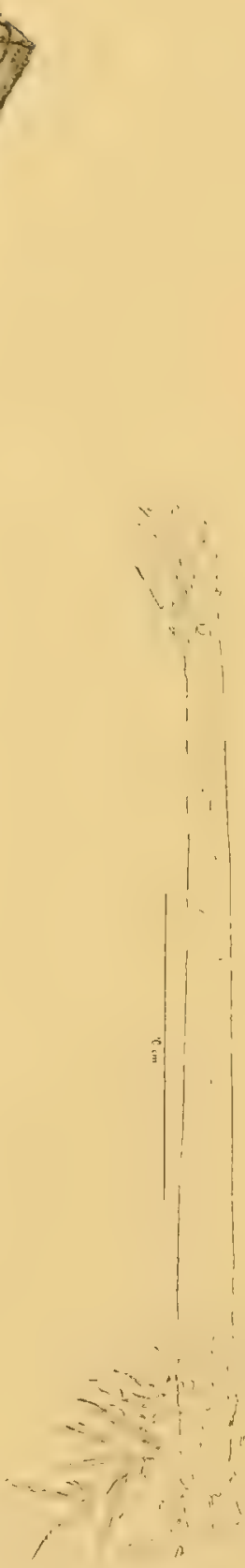

Fig. 108 .

Fig. 107-108. Barrandeina Dusliana, (Kír.) Stur.

107. Srbsko. (Univ. Wien.) Un fossile a) avec sa contre-empreinte. b) Dichotomie. Aspidiaria $\frac{1}{1}$.

108. Srbsko. (K, k. geol. Reichsanstalt.) Appendices basilaires. Tinorria 3.

au plus de long, sur 2-8 de large, et très rapprochées les unes des autres, jusqu'à se toucher (fig. 108-117, 120, 121). Peut-être avons-nous dans certaines figures de véritables Knorria (fig. 108, 111, etc.). 
Mais dans le type le mieux caractérisé que nous ayons (avec sa contre-empreinte), fig. 107, nous voyons qu'il s'agit de forme correspondant tout à fait à l'état de conservation qu'on appelle Aspidiaria chez les Lépidodendracées (voir Potonié Silurflora, p. 117, fig. 72, à propos de Lepidodendron Veltheimi).

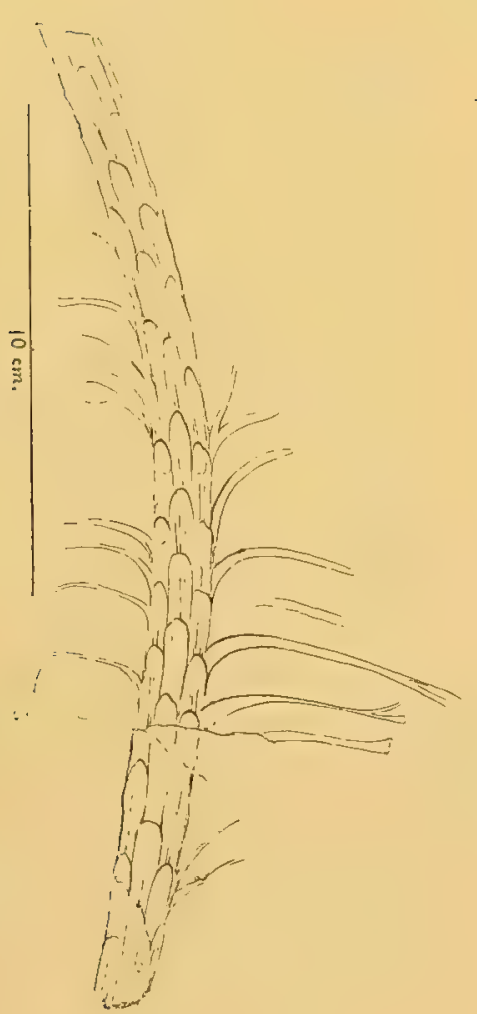

Fig. 109.

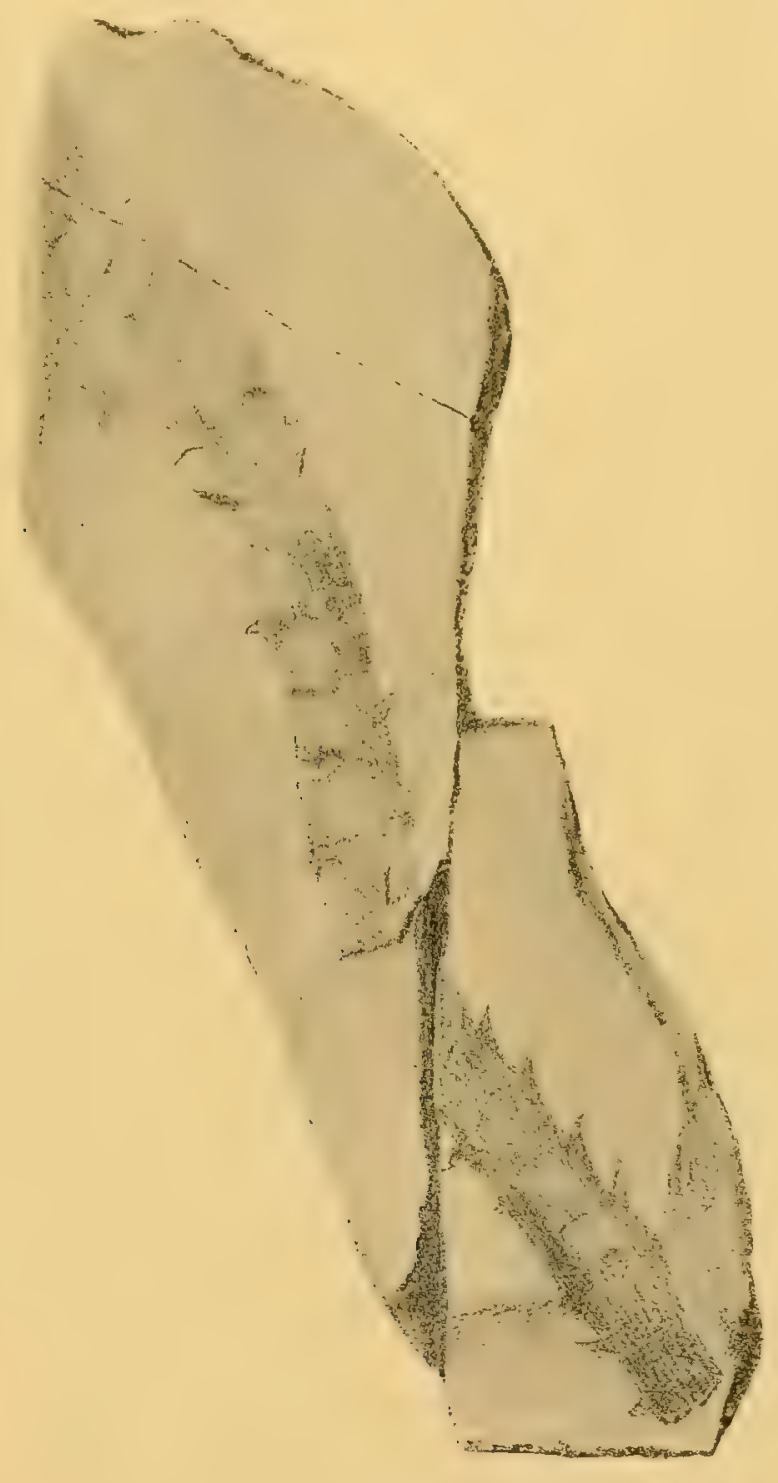

Fig. 110.

Fig. 109-110. Barrandeina Dusliana, (Krr.) Stur.

109. Srbsko. (K. k. deutsche technische Hochschule, Prag, la contre-empreinte chez M. Dusl à Beraun.) Knorria, feuilles petiolées, lobées au sommet. Nervation $\frac{1}{2}$.

110. Hostim. (K. k. geol. Reichsanstalt.) Knorria, base des feuilles $\frac{1}{1-}$

On peut très nettement dans la contre-empreinte (vue de l'intérieur du tronc) constater la présence de "lentilles" plus ou moins allongées. Ces "lentilles" sont les moulages de coussinets vus de dedans, sous les tissus périphériques, le centre de la tige ayant disparu. Ce type était très clairement un semblable moulage de l'intérieur des coussinets foliaires. 


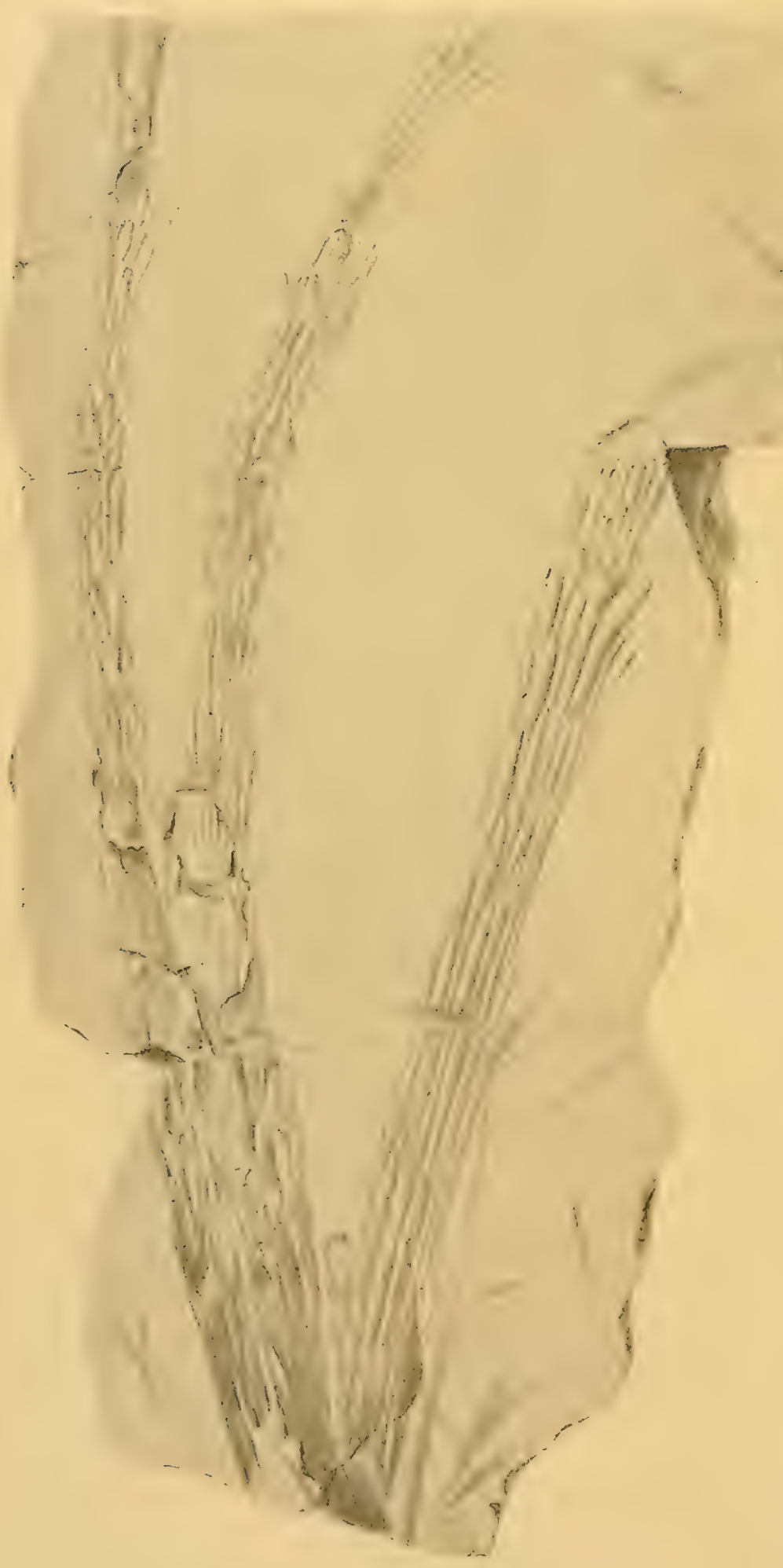

Fig. 111. Barrandeina Dusliana, (Kir.) Stur.

111. Srbston. (K. k. deutsche techn. Hochschule, Prag.) Ixe deux fois dichotomisé. lnorria. Feuilles ginkgoîdes (avec nervures dichotomiques?) $\frac{1}{2}$.
Dans un ou deux de nos échantillons, nous avons rencontré. à la base de la tige, des appendices étranges que nous n'avons pas su comment interpréter (racines? débris de feuilles?) (fig. 108).

Nous avons considéré ces fossiles comme pouvant être des restes de Ginkgoacées à cause de la présence sur plusieurs de nos tiges, de feuilles, ou plutôt d'empreintes bien caractérisées comme restes foliaires (fig. 109, 111, 112), *) et rappelant de très près les feuilles de certaines Ginkgoacées fossiles. Ces feuilles, dont les morceaux étaient malheureusement bien petits, devaient avoir été plusieurs fois et assez profondément découpées, et inontraient encore des nervures ${ }^{* * *}$ ) très nettes, et çà et lir dichotomiques (fig. 111, 112). D'autres, très étroites, se présentaient comme un pétiole terminé par un petit morceau de limbe avec la lobation caractéristique

*) Notre fig. 112 se retrouve sous une apparence un peu différente chez Stur (Pl. V, fir. 8) sous le nom de Barrandeina Dusliana. Il ne faut pas trop s'étomer si la photographie de Stur est plus nette que notre dessin, car nous avons pu constater sur l'original que les empreintes étaient colorées à la sépia, et après avoir lavé l'échantillon, il nous est apparu tel que nous le représentons.

**) En allemand, nous n'emploierions pas le mot "Nerven" qui ne nous semble pas justifié. De tous les mots allemands utilisés pour désigner les éléments conducteurs, nous préférons employer le mot "Adern". En français, nous utiliserons le mot mervures", qui n'a pas les mêmes inconvénients que le "Nerven" allemand. 
(fig. 109). Comme le montrent les empreintes knorrioïdes et les bases foliaires de certains échantillins;, les feuilles devaient être nombreuses tout autour de la tige et présenter une disposition spiralée.' 'Un

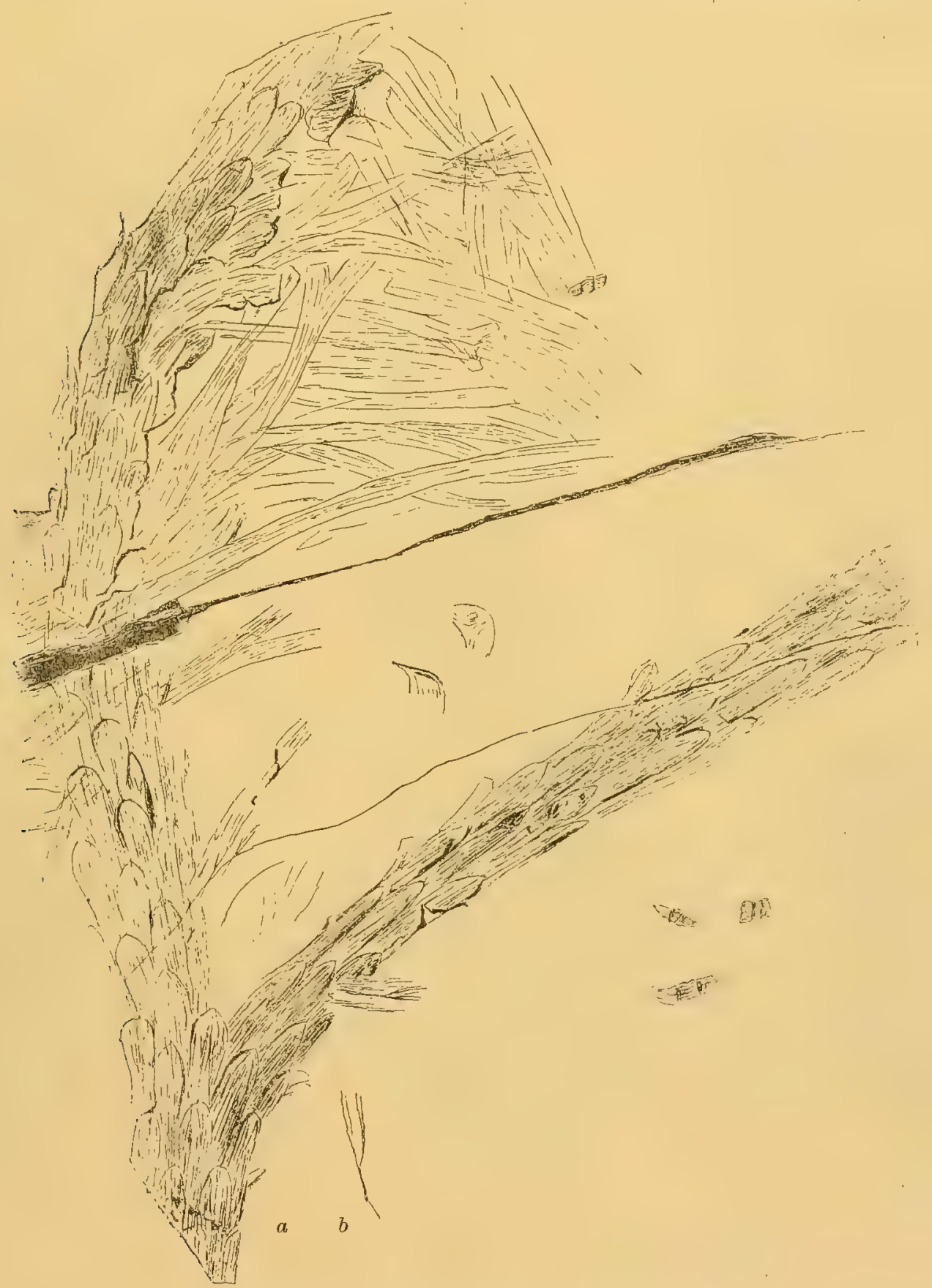

Fig. 112. Barrandeina Dusliana, (Kr.) Stur.

112. (Collection Dusl.) Original de Stur: Pl. V, fig. 8. Axe dichotomisé, Knorria, feuilles dichotomes avec nervures. Empreintes de Trilobites $\frac{1}{1}, b$ ) une nervation grossie 2 fois.

échantillon surtout était très caractérisé par les marques knorrioïdes en disposition spiralée, et les bases des feuilles (fig. 110). Notre fig. 118 montre bien le rapport entre les feuilles et les Knorria; 
les bases des feuilles que porte encore cet axe sont très nettement dans la continuation des empreintes knorrioïdes.

2. Nous avons trouvé en outre une empreinte foliaire très nette (fig. 122) assez grande $\left(2^{\mathrm{cm}}\right)$, lobée à la façon des feuilles de Ginkgoacées fossiles, à nervures parallèles, se dichotomisant à la

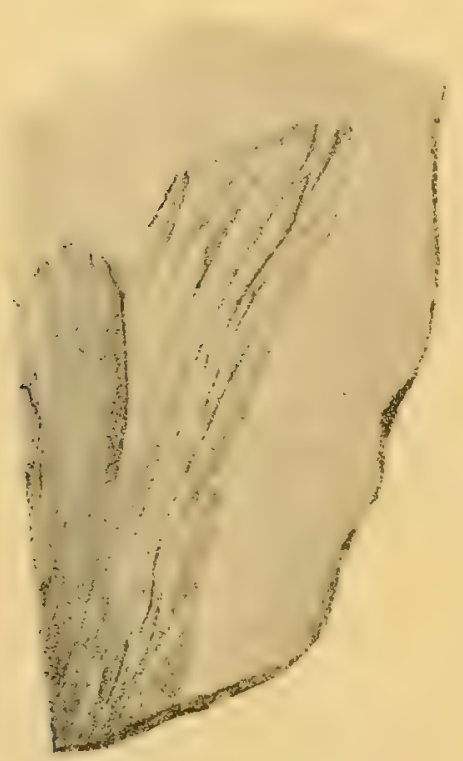

Fig. 113.

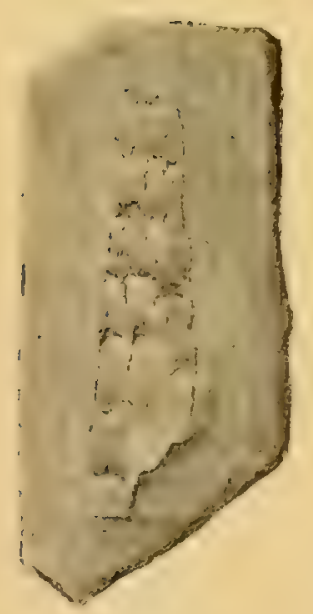

lig. 116.

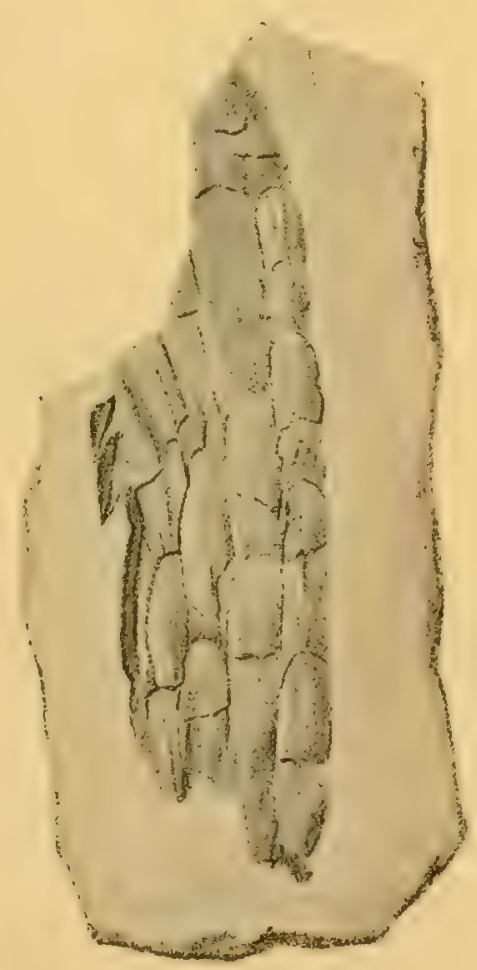

Fig. 114 .

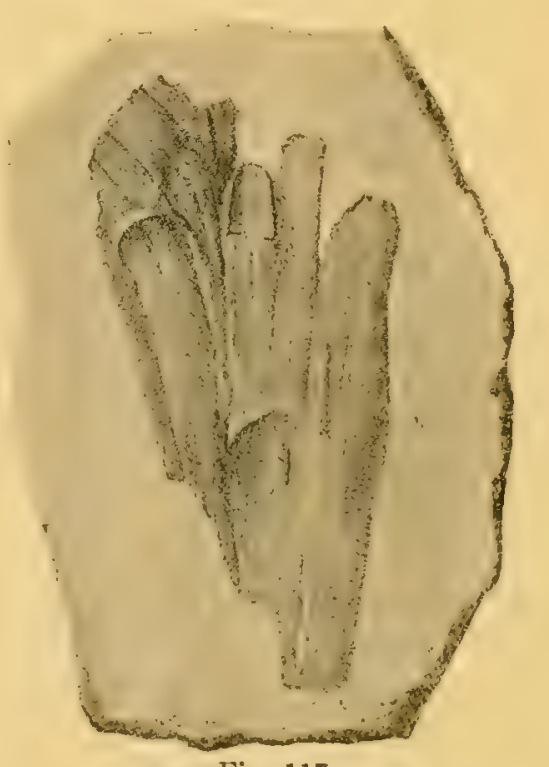

Fig. 117.

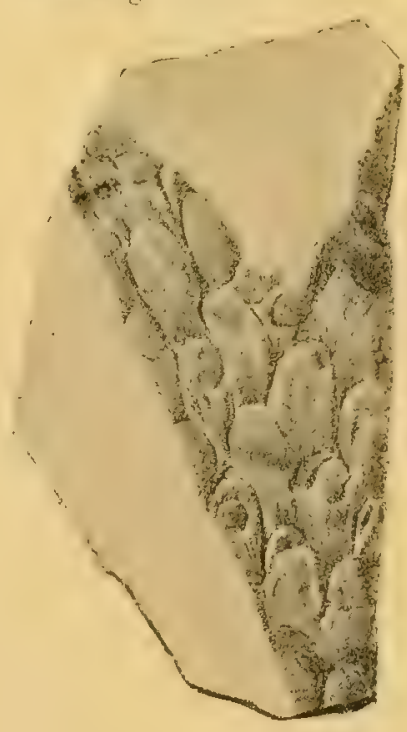

Fig. 115.

Fig. 113-117. Barrandeina Dusliana, (Kr.) Stur.

113-116. Hostím. (K. k. geol. Reichsanstalt.) Knorria très larges
117. Srbstio. (,$~$

base des échancrures. Cette feuille ne rappelle pas absolument les débris foliares décrits ci-dessus mais ceux-ci sont en si petits fragments quil est probable que tous ces restes appartiennent quand même à une seule et même espèce.

Quant au: nom à attribuer à ce fossile, la question se pose comme pour les précédents. 
Krejči avait appelé ces débris Protolepidodendron Duslianum, Kr.

Stur les considera comme des "Characere precursores", sous le nom de Barrandeina Dusliana, (Kr.) Stur. Le premier de ces noms ne peut être conservé malgré la priorité, attendu qu'il aurait l'air de vouloir rappeler une parenté de notre type avec les Lépidodendrées. Nous conserverons le nom de Barrandeina, quand même Stur en a fait une Algue, et parce qu'il n'y a pas, dans la nature actuelle, d'Algues de ce nom.

Les dessins figurés par Stur et ses originaux, que nous avons eus entre les mains, ne laissent aucun doute sur l'identité des pièces de Stur avec les nôtres. Stur a indiqué dans ses dessins des appendices foliacés qu'il a interprétés comme lobes du thalle et que nous considérons comme les restes des feuilles; il est curieux qu'il n'ait pas arrêté davantage son attention aux empreintes knorrioïdes et aspidiarioïdes qu'il figure et qui auraient dû l'éclairer sur la nature de ces restes et les lui faire considérer comme ayant appartenu à des plantes supérieures et non à des „Characeœ precursores".

Krejči cite, à Srbsko, des impressions de feuilles rappelant, dit-il, Cordaites borassifolius, Corda, mais qui pourraient être parentes à des Delesserites. D'autre part, au Musée de Bohême, nous avons vu, déterminées comme Flabellaria borassifolia, Stbg. (= Cordaites borassifolius), des empreintes que nous avons pu reconnaittre par les bases des feuilles comme étant analogues aux

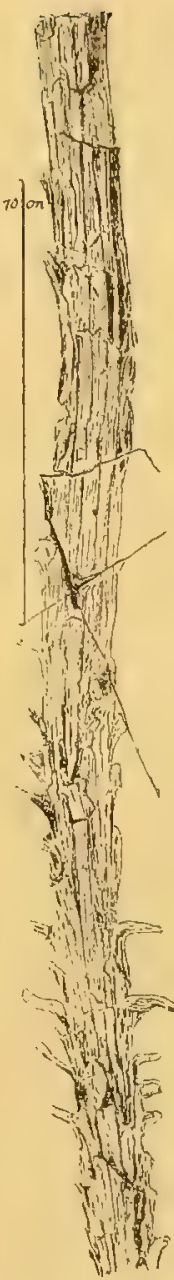

Fig. 118.

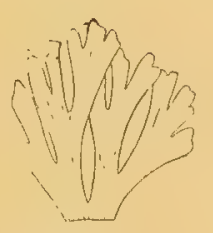

Fig. 122.

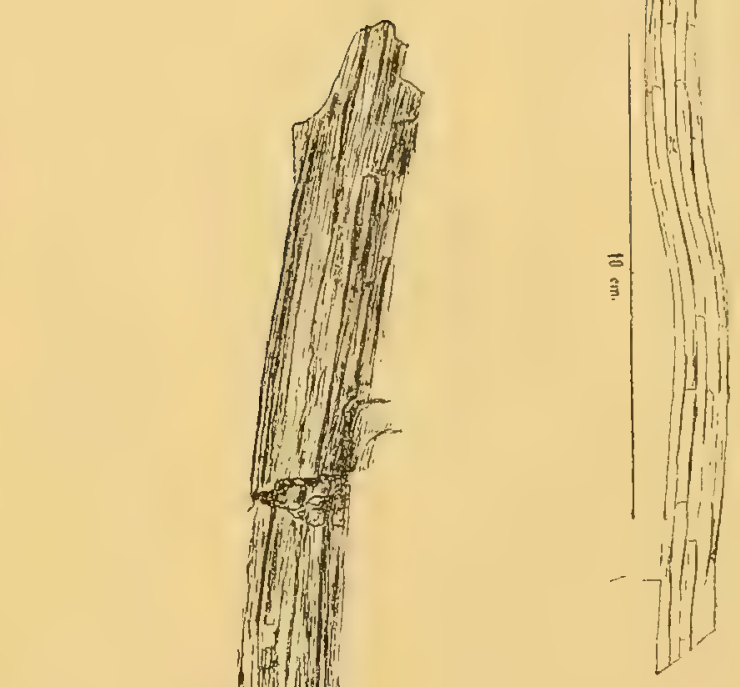

Fig. 120.

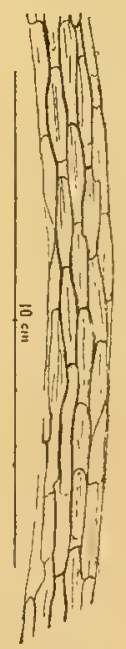

Fig. 121.

Fig. 118-122. Barrandeina Dusliana, (Kr.) Stur.

118. (Collection Dusl.) Knorria, bases foliaires. Env. $\frac{1}{2}$.

119. Srbsko. (Geol, Landesmuseum, Berlin.) Axe central. $\frac{1}{1}$.

120. " (Böhm. Univ. Prag.) Knorria $\frac{1}{2}$.

121. " (Deutsche Univ. Prag.) Knorria, traces foliaires au sommet des Knorria $\frac{1}{2}$.

122. $\rightarrow$ (Wien. Reichsanstalt.) Feuille, nervures t.

échantillons décrits par nous sous le nom de Barrandeina; il est probable que c'est à ce groupe aussi que se rattacheraient les Cordaites signalés par Krejči à Srbsko. Nous en voyons une preuve 
de plus dans le fait que Dawson a figuré (1862/63) une forme très analogue à notre Barrandeina: il Ia considère comme Cordaites douteux. Il n'y aurait rien d'extraordinaire à trouver des Ginkgoacées dans ces terrains très anciens, puisque Nathorst. dans ses "Polarländer", cite, comme caractéristique du Dévonien de l'Ile des Ours, une feuille qu'il désigne sous le nom de Psygmophyllum Williamsonii, Nath., et qüil ne serait pas éloigné de considérer comme un reste de Gymmosperme, vu l'analogie des découpures et des nervures de cette feuille avec celles des feuilles de Ginkgoacées. "On désigne, dit-il, de semblables restes sous le nom plus récent de Ginkgophyllum, Sap., mais je. "préfère leur conserver le nom de Psygmophyllum, qui n’affirme rien quant à la situation systéma"tique de ces débris." Dawson, au Canada, décrit un Cyclopteris qui parait être un Givizyophyllum. On a l'habitude de mettre parmi les Ginlogoacées ces débris de feuilles dénommés Ginkgophyllum, mais il est difficile dans ces restes dévoniens d'affirmer à quel genre de Ginligoacée fossile il faudrait rattacher les débris en question, car la présence des organes fertiles seule pourrait élucider cette question. Aussi considérons-nous ces types comme voisins des Psygmoplyyllum, tout en leur laissant le nom de Barrandeina et en rappelant que leur nervation et leur apparence générale pourraient faire penser aussi aux Baiera.

Il est intéressant de constater la dichotomie de la tige de cette Ginkgoacée, car, chez le type actuel, la dichotomie a disparu de la tige et ne se rencontre plus que chez les fenilles; c'est naturel, puisque c'est la tige tout d'abord qui devait s'adapter aux fonctions mécaniques de soutien. La dichotomie du tronc étant défavorable à ce point de vue, la plante a modifié sa disposition générale, tandis que le caractère ancestral a été conservé dans les feuilles seulement. Et comme d’autres détails inexplicables des feuilles de certaines plantes actuelles ont été éclaircis lorsqu'on eut trouvé les ancêtres fossiles de ces plantes, et qu'ou eut pu interpréter ces caractères comme ancestraux, de même il était intéressant de signaler une Ginkgoaće dont la tige présenterait ce caractère qui ne s'est conservé que dans les feuilles du type actuel.

Ces types fossiles de Ginkgoacćes étaient intéressants à un autre point de vue: c'est que In disposition de ces feuilles, comme on peut la voir représentée dans nos figures, s'explique le mieux par la théorie du "Pericaulom" (Potoniẻ, 1903). On voit en effet la base de ces feuilles et la prolongation dans la tige de leur faisceau entouré da moulage parychnoïde, envelopper comme d'un manteau la partie centrale de la tige; cette disposition, comme du reste les Knorria, la marche des faiscenux, les Aspidiaria et bien d'autres faits intéressants seraient autant d'arguments presque tangibles qui parleraient en faveur de cette théorie.

\section{Incertie sedis.}

Nous avons à décrire ici des types incertains qui, par quelques caractères, peuvent se rattacher à notre Barrandeina.

10. Un axe assez peu clair, portant d'indistinctes Knorria, mais intéressant par li terminaison des ramifications minces, couvertes de feuilles longues et nombreuses (fig. 123). Ce seraient peut-être des fleurs, mais les empreintes sont si peu nettes que nous ne pouvons l'affirmer. La présence des bourrelets linorrioïdes seule nous a autorisés à le placer dans le voisinage de notre Barrandeina.

20. Un axe intéressaut (fig, 124), qui montrait des ramifications latérales et où l'on pouvait distinguer assez nettement les parties que l'on conmaît chez les Calamites, à savoir: au centre, une moelle (m), autour de laquelle se trouve le bois (b), et, à la périphérie, l'écorce (í). Nous avons placé ici cet échantillon, car il nous a semblé voir dans nos figures 117 et 118, en des endroits d'où les Knorria avaient été enlevées, une disposition assez semblable à celle que nous signalons ici, mais moins nette.

3", fig. 125 et 126. De larges axes avec de très larges Knorria. 


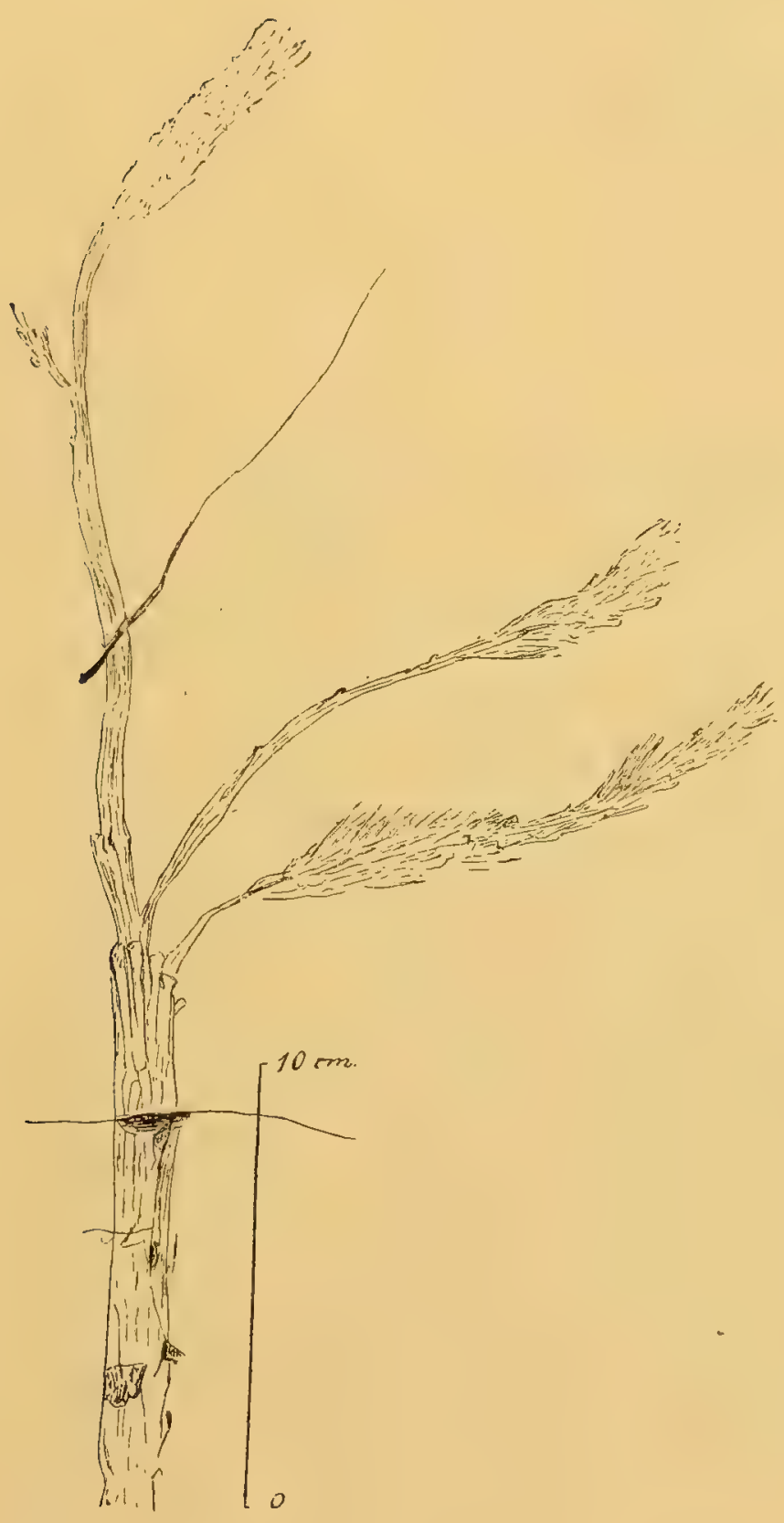

Fig. 123.

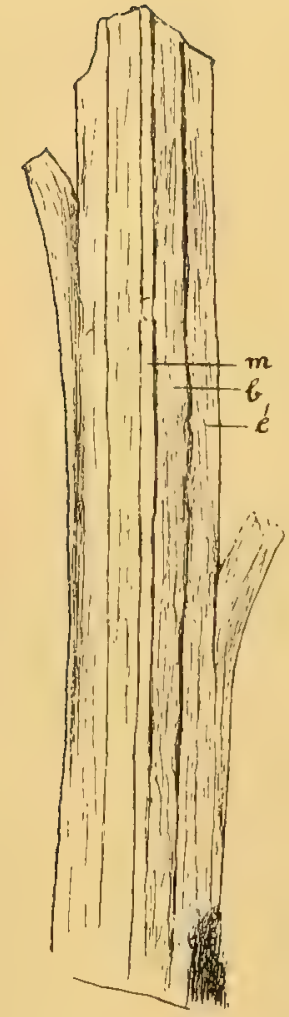

Fig. 124.

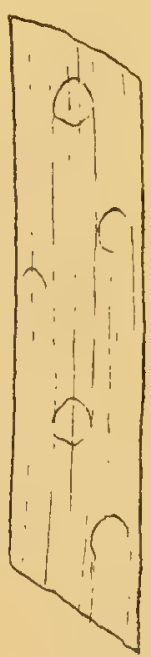

Fig. 126.

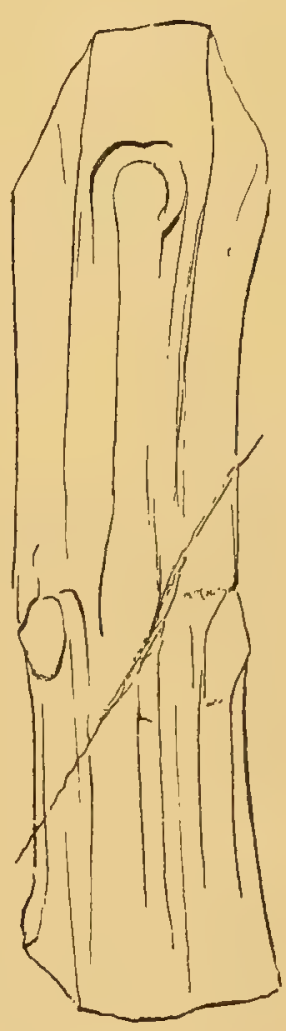

Fig. 125.

Fig. 128-126. Incerta sedis.

123. (Collection Dusl.) Axe ramifié. Knorria. Branches feuillées (fleurs?) $\frac{1}{2}$

124. Srbsko. (Geol. Landesmuseum, Berlin.) Axe avec ramifications. é =écorce, $b=$ bois, $m=$ moelle $\frac{1}{1}$. 125-126. $\quad n \quad$ (Reichsanstalt, Wien) Axes, Knorria? $\frac{1}{\uparrow}$.

Ces trois types sont' en trop maurais état pour quil soit possible, soit de les rattacher définitivement à un genre connu, soit d'en établir une diagnose suffisamment détaillée quỉ permettrait de leur attribuer un nom nouveau. 
Psilopleyton spinosecm, (Kr.) P. et B.

Fig. 127-139

et Psilophyton bohemicam, (Stur) P. et B.

Fig. $142-147$.

Fucus Nováki, Stur.

Haliserites spinosus, Kr. ex parte.

Protolepidodendron Duslianum, Kr. ex parte.

Lessonia bohemica, Stur.

Sargassites, Ettingsh. (in Stur).

Barrandeina Dusliana, Stur ex parte.

Pour le moment, nous considérons comme pouvant être des restes de Conifères des empreintes assez régulièrement disposées, mais qui n'ont malheureusement pas pu nous fournir beaucoup de renseignements sur les plantes auxquelles elles ont appartenu.

Nous trouvons ces débris décrits sous les noms de Lessonia bohemica, Fucus Nováki, Barrandeina Dusliana, Protolepidodendron Duslianum, Haliserites spinosus, Sargassites sp., et cette diversité de noms prouve, sans qu'il soit besoin d'insister davantage, le peu de précision de ces empreintes et la difficulté qu'elles présentent à une exacte interprétation. Quant au nom à leur attribuer, nous aurions voulu leur maintenir un des noms ci-dessus pour satisfaire aux lois de la nomenclature; mais les noms des genres vivants Lessonia et Fucus et du genre fossile Haliserites ne pouvaient nous convenir, puisque nous ne sommes pas en présence d'Algues; le nom de Protolepidodendron aurait l'air de vouloir attribuer à nos débris une parenté avec les Lépidodendracées, ce que nous ne saurions affirmer. Quant à Barrandeina, il ne nous satisfait pas non plus, car il a été plus généralement appliqué à des restes de toute autre apparence, dont nous avons parlé plus haut.

Nous devons relever, à propos de ces échantillons les analogies qui existent entre eux et certaines formes du genre si peu délimité et si hétérogène que Dawson a décrit sous le nom de Psilophyton. Dawson figure, dans ses "fossiles du Dévonien et dı Silurien supérieur du Canada", des formes qui rappellent d'assez près les nôtres par l'axe, porteur de feuilles plus ou moins développées. Il est vrai que Dawson dessine sur les trones des points, tandis que les marques que nous avons relevées sont plus généralement des stries transversales. Mais ce caractère, vu l'état défectueux des fossiles, ne peut guère être pris en considération comme un fait important. Certains Arthrostigma décrits par Dawson dans le même travail présentent également des analogies avec les formes qui nous occupent. Dawson mettait le Psilophyton princeps parmi les Psilotacíes, mais cette interprétation n'est rien moins que certaine, et la reconstitution qu'il a donnée de cette plante est établie sur des bases trop problématiques pour que l'on soit tenu de s'y rattacher. Du reste, Dawson semble mettre dans ce genre un peu toutes les formes qu'il ne sait à quel type rapporter. Jusqu'ici nous ne savons guère de certain sur le genre Psilophyton que ce qui résulte des recherches de Solms. (Voir aussi Potoniẻ, qui a résumé cette question dans son Lehrbuch, p. 263. - Voir encore Dawson, 1862-1863, entre autres p. 16.)

Le Psitophyton grande de Penhallow, qui pourrait rappeler vaguement certaines de nos formes, ne parait s'approcher que de très loin du genre Psilophyton, Daws.

En 1895, Palacký s'occupe de cette question. Après avoir constaté les difficultés qu'offre l'étude des restes du "Silurien supérieur (ou Dévonien), maintenant appelé ordinairement Hercynien," de Hostím, et avoir décrit ces fossiles comme des branches ayant séjourné longtemps dans l'eau avant d'être pétrifiées dans la vase, il relève le fait qu'on n'a pas encore appliqué à ces fossiles les méthodes modernes de recherches, par exemple l'emploi du microscope au moyen de coupes minces. Quant aux 


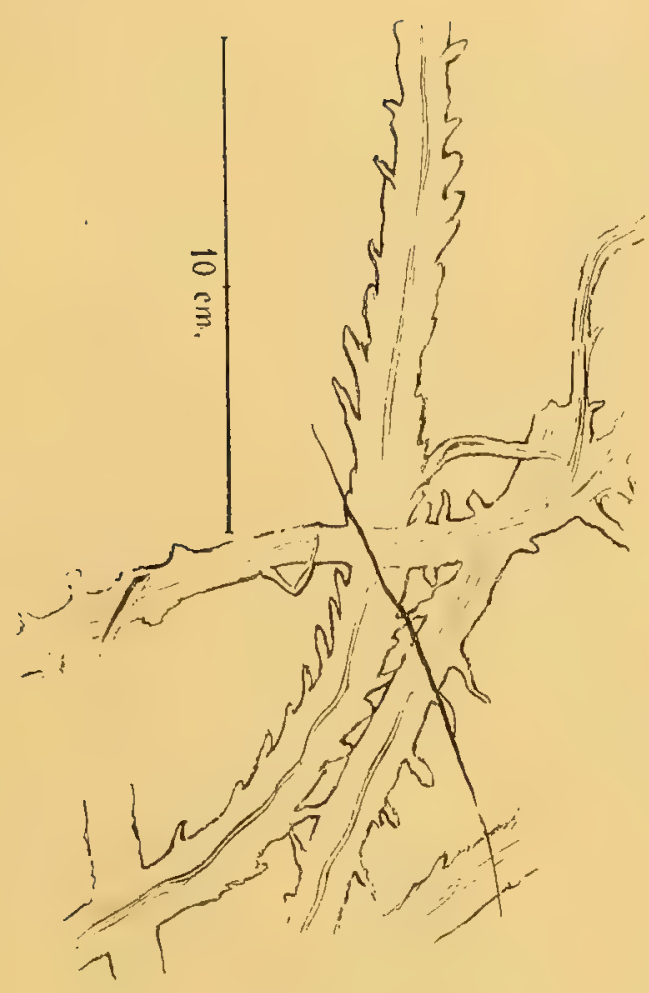

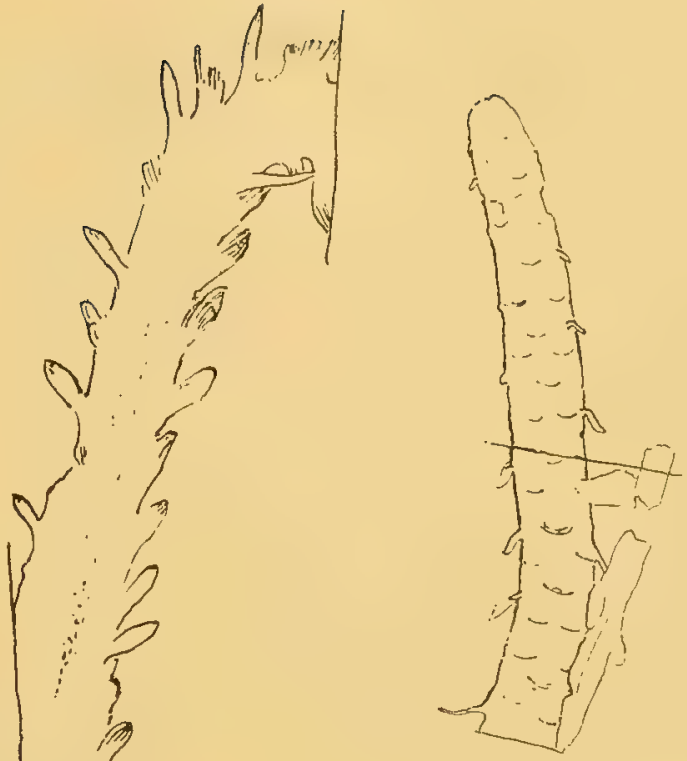

Fig. 128.

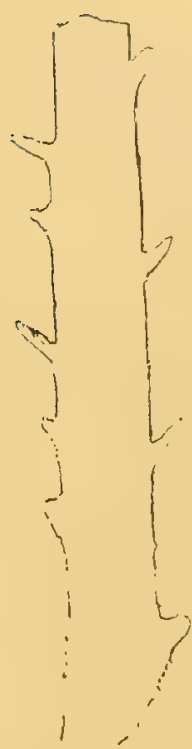

Fig. 130.

Fig. 127.

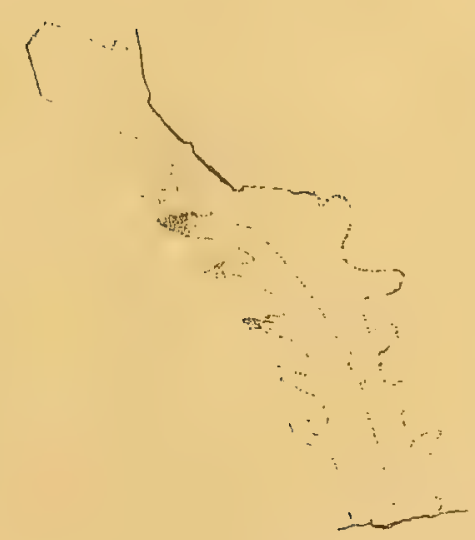

Fig. 131.

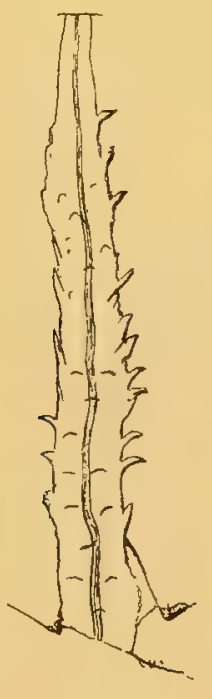

Fig. 133.

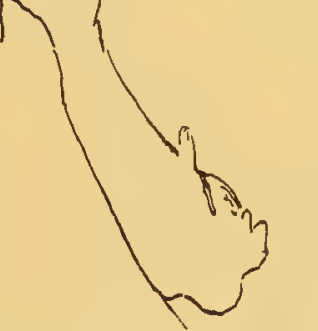

Fig. 129.

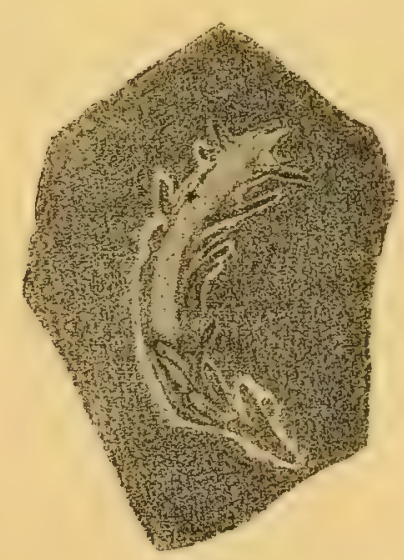

Fig. 132.

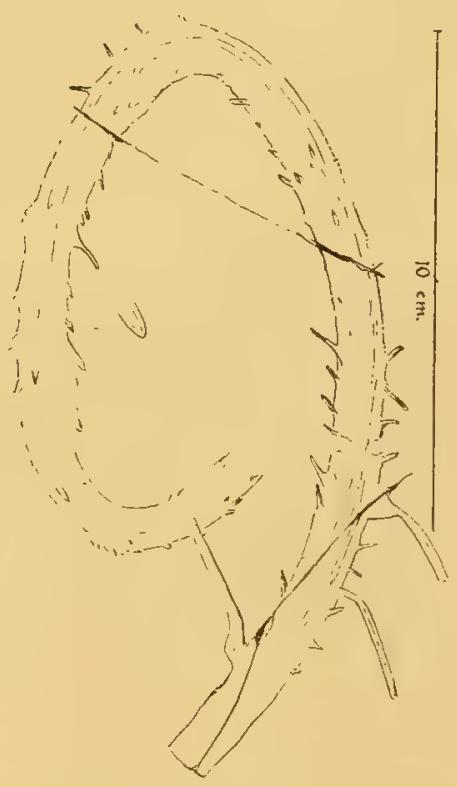

Fig. 134.

Fig. 127-134. Psilophyton spinosum, (Kr.) P. et B.

127. Axe central, dichotomie. Rameaux avec feuilles latérales $\frac{1}{2}$.

I28. Srbsko. (K. k. d. Univ.. Prag.) Feuilles cicatrices transversales disposées en spirales $\frac{1}{\mathrm{l}}$.

129. Hostim. (K. k. d. Univ., Prag.) Rameau feuillé $\frac{1}{1}$.

130. Srbsko. (K, k. d. techn. Hochschule, Prag.) Rameau feuillé $\frac{1}{1}$.

131. $"$ (Böhm. Univ. Prag.) Rameau feuillé. Infiltrations minérales $\frac{\mathrm{I}}{1}$

132. Hostím. (K. k. geol. Reichsanstalt.) Rameau feuillé $\frac{1}{1}$.

133. Srbsko. (Deutsche Univ. Prag.) Cicatrices transversales, axe central $\frac{1}{1}$.

134. Hostim. (K. k. d. Univ. Prag.) Axe, feuilles latérales et superficielles, appendices basilaires curieux $\frac{1}{2}$; au centre du dessin, une feuille montrant 2 lignes (nervures?) $\frac{1}{1}$. 
déterminations de Stur, il lui a transmis personnellement ses doutes à ce snjet en lui envoyant un petit morceau de fin charbon de Hostím, sans que Stur lui ait fait quelque opposition. C'étaient, ajoute Palacký, de grandes Algues atlantiques (Borya pour la plupart) qui avaient conduit Stur à sa théorie des Algues de Bohême, et le fait qu'il n'était pas botaniste laisse comprendre ses erreurs; l'auteur contin 1 : „Le premier qui fit une supposition positive sur l'identification de ces vieilles "et intéressantes plantes, fut le Professeur Dawson à Montréal, Canada, dans son livre ,Geological „history of Plants' (1888, London)." Après avoir cité l'opinion de Dawson, Palacký ajoute: „Je lui "écrivis en lui envoyant un exemplaire de Hostinella, qu'il reconnut dans ume lettre comme Psilo„phyton... Dernièrement Penhallow publia, comme trouvées dans l'Erian (Dévonien) de New"York et de Pensylvanie, des formes rappelant celles de Bohême et qu'il décrit comme Haliserites, "Dictyotites, Psilophyton." Et Palacký conclut: "Avant tout, il est nécessaire d'échanger des maté"riaux entre les fossiles américains et bohêmes, car une analyse microscopique et chimique s'impose "avant qu'on puisse définitivement parler de ces intéressantes vieilles plantes."

Dans „l'Histoire gréologique des Plantes“ (Dawson) nous lisons (page 39): „Quoique je n'aie pas „vu les spécimens, je ne puis douter que les plantes du Silurien de Bohême fou la plupart d'entre nelles du moins), décrites par Stur comme des Algues et des Characées ne soient réellement des „plantes terrestres et notamment le genre Psilophyton. Je puis dire aussi que les empreintes aplaties "de Psiloplayton et d'Arthrostigma du Silurien supérieur et de l'Erian de Gaspé auraient pu être "lécrites comme Algues, si lon n'avait retrouvé dans quelques-unes l'axe fasciculaire avec des "vaisseaux scalariformes (barrerlessels) bien conservés."

Le travail de Palacký ne comportant pas de dessins, il nous est impossible de savoir laquelle des nombreuses formes décrites ou déterminées par Stur comme Hostinella, Dawson a reconnue comme Psilophyton. Nous voulons admettre que cest celle qui ressemble le plus à: ce que l'on considère généralement comme Psilophyton (voyez Dawson et Solms-Laubach), c'est-à-dire un axe avec des appendices de chaque côté. Nous ne voyons pas d'inconvénient à rattacher les formes qui nous occupent au genre Psilophyton, d'autant plus que ce genre est très raste et quil ne peut être considéré que comme un groupement provisoire d'espèces vagues; nous nous appuyons sur les données de Solms, qui veut qu’à ce genre soient rattachés les axes avec des appendices latéraux qui leur ajonnent une apparence très spéciale et qui ont poussé Stur à les interpréter comme des thalles dentés. Mais nous n'irons pas si loin que Dawson, qui fait de Psilophyton une Psilotacée, ni que Zeiller, qui prétend (p. 368) que ce pourrait être un type mixte entre les Fougères et les Lycopodiacies. Au contraire, nous répétons que nous ne savons pas où ranger les deux formes que nous nommons, d'après les noms spécifiques de Stur, Psilophyton spinosum et $P$. bohemicum.

Nous avons dit, plus haut, qu'on pourrait les considérer comme des Conifères, parce qu'en effet elles ont certains caractères qui autorisent cette supposition, mais cela non plus ne peut être affirmé, et, ici comme ailleurs, nous devons rester dans l'incertitude en attendant la découverte de documents meilleurs.

Stur réunissait nos deux types sous deux noms:

10. Lessonia bohemica, Algue brune de l'ordre des Laminariées, et les empreintes qu'il avait sous les yeux lui rappelaient les organes végétatifs de ces Algues.

20. Fucus Nováki, une Fucacée.

Ici encore nous n’avons pas bien saisi les caractères sur lesquels Stur s'est appuyé pour diffé-: rencier les fossiles décrits sous ces deux noms. Il dit en outre (p. 341), à propos de Lessonia bo- . hemica: „Quant à la signification de ces restes, je dois remarquer d'abord que v. Ettingshausen „expliqua comme un reste de Fucacée (Sargassites) l'échantillon de Hostín que Barrande lui arait ${ }_{n}$ communiqué en 1865. Pour moi, je dois dire que je le tiens pour proche parent de Lessonia .fuscescens, c'est-à-dire pour une Laminariée." Stur représente ses deux types comme étant dentés. 
au bord. Il est à remarquer que l'apparence donnée à ces fossiles, apparence qui peut rappeler, en effet, parfois celle des Phrophycées, provient souvent d'infiltrations minérales (de fer probablement),

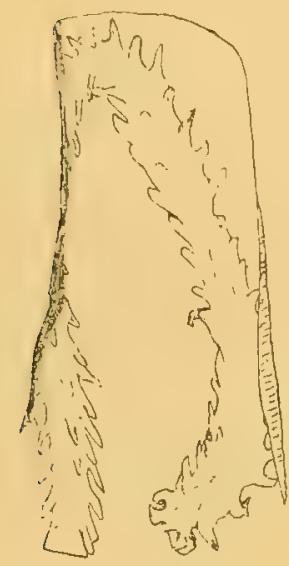

F.g. 135 . it

sis

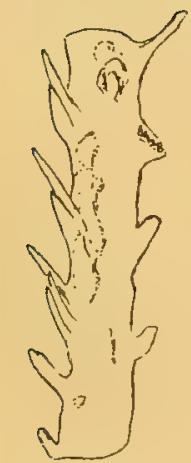

Fig. 136.

Fig. 133.
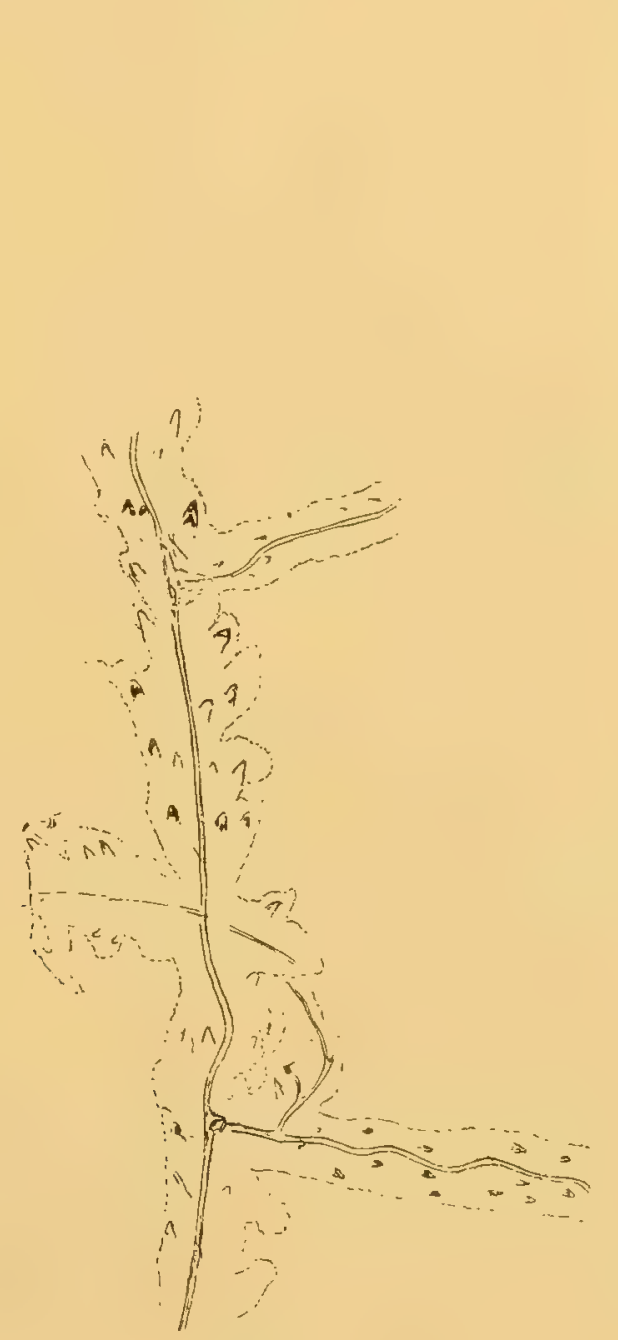

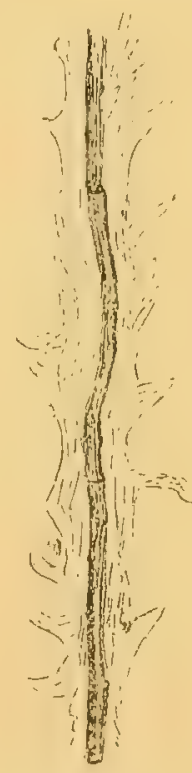

Fig. 139.

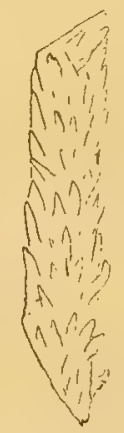

Fig. 140.

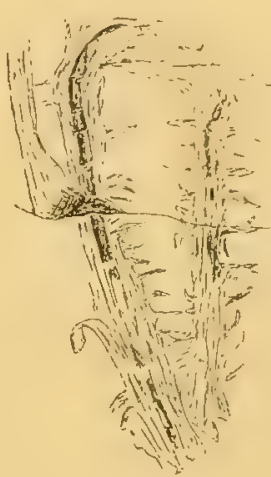

Fig. 137.

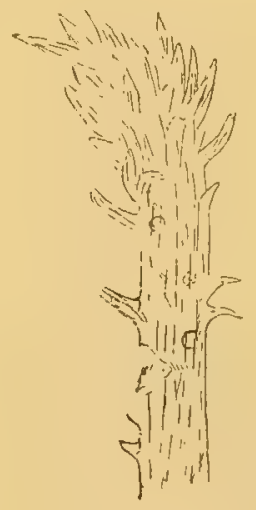

Fig. 141.

Fig. 135-139. Psilophyton spinosum, (Kr.) P. et B.

135. Hostím. (Univ: Wien.) Axe feuillé $\frac{1}{2}$

136. Srbsko. (Univ. Wien.) Axe feuillé $\frac{1}{1}$.

137. $\quad$ (Geol. Landesmuseum, Berlin.) Rameau feuillé, axe central $\frac{1}{2}$

138. " (Collection Dusl.) Axe central dont on saisit bien les ramifications. Petites feuilles sur toute la surface. Infiltrations $\frac{1}{1}$.

139. $\pi$ (Geol. Landesmuseum, Berlin.) Rameau feuillé, axe central ł.

Fïg. $140-141$. Incerta sedis.

140-141. Srbsko. (Geol. Landesmuseum, Berlin.) Rameaux couverts des nombreuses grandes feuilles disposées en spirales. $\frac{1}{2}$.

qui font alors apparaitte comme un double contour, et peuvent, en tous cas, en masquant les limites réelles du fossile, empêcher d'en saisir les détails caractéristiques. 
I. Nous avons groupé sous le nom de Psilophyton spinosum tous les fossiles qui nous présentaient les caractères suivants: Des espèces de rameaux pourvus, sur plusieurs de nos échantillons, d'un axe rasculaire central, assez mince, vu la largeur des tiges, mais très distinct (fig. 127, 133. 137-139). La surface des rameaux portait des cicatrices de feuilles (fig. 127-139). Lorsque ces cicatrices se trouvaient sur les côtés de la tige, elles ont laissé une empreinte latérale (fig. 129-131), qui a pu faire croire aux dents d'un thalle dans les échantillons mal conservés, voilés par le précipité minéral, ou, ce qui est plus fréquent encore, grossièrement colorés à la sépia. Mais en y regardant de plus près, les pièces mieux conserrées et soigneusement nettoyées montraient souvent des cicatrices foliaires, distinctes sur toute la face de la tige, et nettement disposées en spirale (fig. 128, 133, 135, 138). C'est ce caractère qui démontre surtout que ce sont des plantes supérieures et non des Algues, et qui nous a fait penser à des Conifères ou à des Lycopodiales, quoique, nous le répétons, nous ne puissions rien affirmer d'absolu. Dans certaines pièces, nous avons pu voir très exactement les feuilles latérales, les soi-disant dents, s'attacher, non pas an bord de la tige, mais sur la face (fig. 129, 133, 135); enfin sur plusieurs de ces tiges, nous arons ru les petites feuilles triangulaires, lancéolées (fig. 134, 135, 138), apprimées contre la tige, et toujours nous avons pu constater la disposition spiralée de ces organes. Ces fossiles venaient de Srbsko et de Hostím.

\section{Incertie sedis.}

Nous décrivons ici un type incertain (fig. 140, 141), qui se rattache à notre Psilophyton spirosum par la disposition spiralée des feuilles; mais ici elles sont grandes et beaucoup plus nombreuses. Elles couvrent toute la surface du rameau, qui, du reste, est en trop mauvais état de conservation pour donner matière à discussion. La fig. 141 montrait une légère courbure du sommet, et par places sur laxe, des empreintes arrondies que nous ne sarons comment interpréter et qui ne sont peut-être que des restes d'animax.

\section{Psilophyton bohemicum, fig. 1.2-147.}

D'autres restes végétaux ont été rencontrés, auxquels les auteurs ont attribué les mêmes noms qu'aux échantillons ci-dessus. L'apparence générale peut souvent être identique à première vue, les mêmes infiltrations de fer, la même apparence de thalle denté pouvant se présenter parfois, ce qui fait comprendre les interprétations de Stur. Mais un examen attentif montre que les feuilles, ici, au lieu d'être disposées en spirale, sont, au contraire, régulièrement placées en verticilles. Nous disions cependant plus haut, que l'on pourrait peut-être rattacher les types spiralés aux types verticillés. C'est qu'en effet nous avons rencontré des échantillons, où il est difficile de dire, si l'on est en présence de l'un ou de l'autre des deux types, et indiquant des relations possibles entre eux.

Des recherches ultérieures seules pourront décider la question de savoir s'il est possible de rattacher les types verticillés aux types spiralés. Cette supposition, étrange au premier abord, n'est pas absurde, puisque nous voyons certaines Conifères, les Cupressinées par exemple, présenter dans leurs états jeunes des dispositions spiralées, et devenir verticillées dans la suite. Les exemplaires verticillés possédaient souvent des stries plus ou moins nettes le long de leurs tiges, rappelant en cela les Spliénophyllacées et les Protocalamariacíes (fig. 144).

Un de nos échantillons nous a montré, après macération de la houille, de très intéressantes ponctuations aréolées, rappelant d'assez près celles de Conifères. Cependant ce point ne devrait pas être pris comme une preuve absolue que nous sommes en présence de Conifères, car cette famille*) n'est pas absolument et exclusivement caractérisée par cette formation, qu'on peut rencontrer chez

*) Voir p. 35 . 


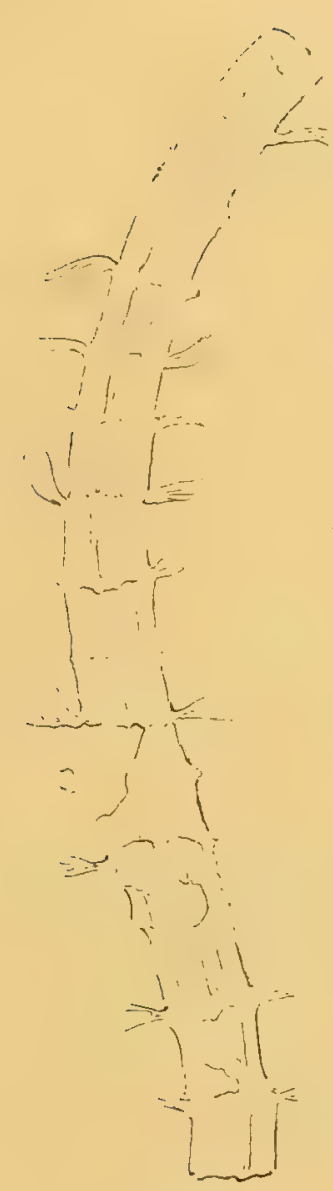

Fig. 144.

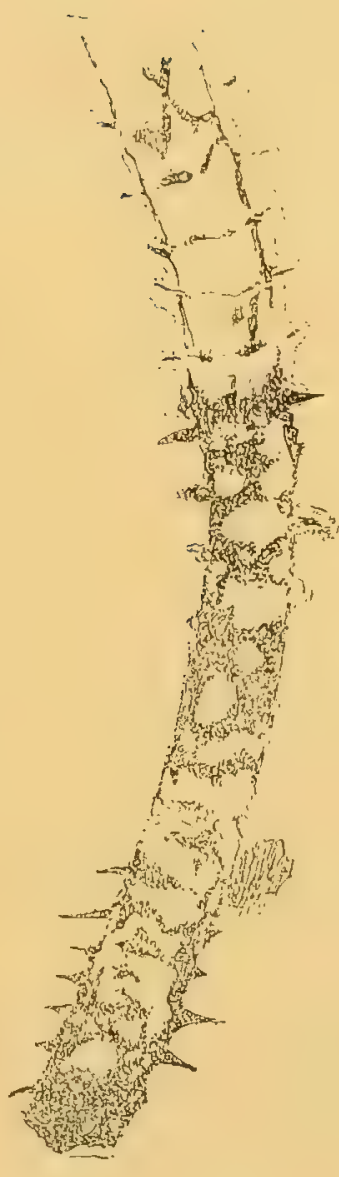

Fig. 145.

Fig. 142-147. Psilophyton bohemicun, (Stur) P. et B,

142. Srbsko. (K. 1. d. Univ. Prag.) Original de Stur, Pl. I, fig. 9. Axe central, feuilles en verticilles $\frac{1}{1}$.

143. Hostím. (K. k. geol. Reichsanstalt.) Rameau feuillé $\frac{1}{1}$.

144. Srbsko. (Böhm. Univ. Prag.) Feuilles verticillées de forme curieuse. Stries longitudinales 1 .

145. " (Böhm. Univ. Prag.) Rameau feuillé. Infiltrations de fer qui font aux feuilles comme un donble contour $\frac{1}{1}$.

146. " (Böhm. Cniv. Prag.) Rameau feuillé. Infiltrations $\frac{1}{1}$

147. Hostím. (Reichsanstalt.) Rameau à feuilles nettement verticillées $\frac{1}{1}$.

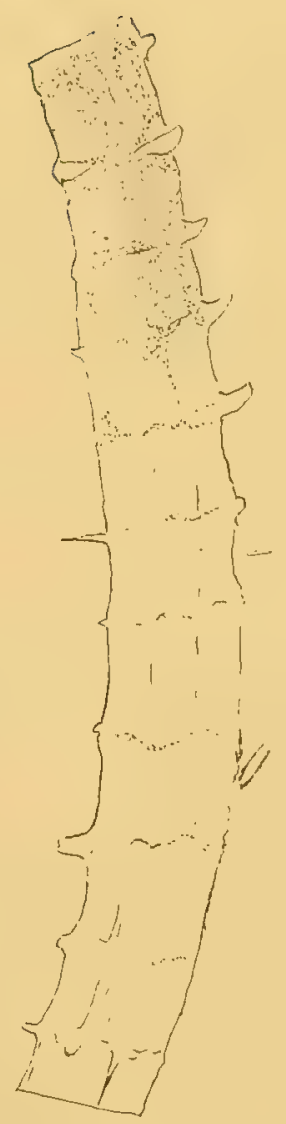

Fig. 146.

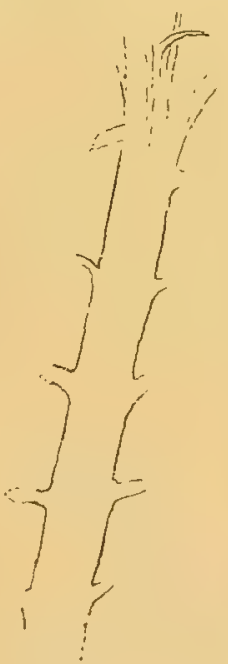

Fig. 143.

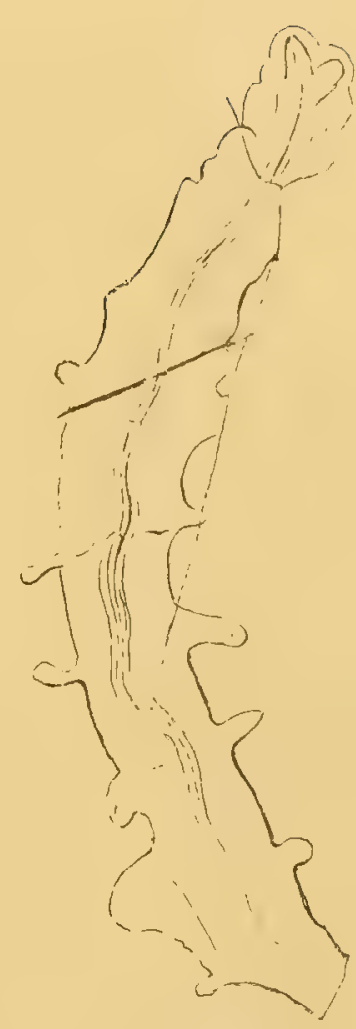

Fig. 14 .

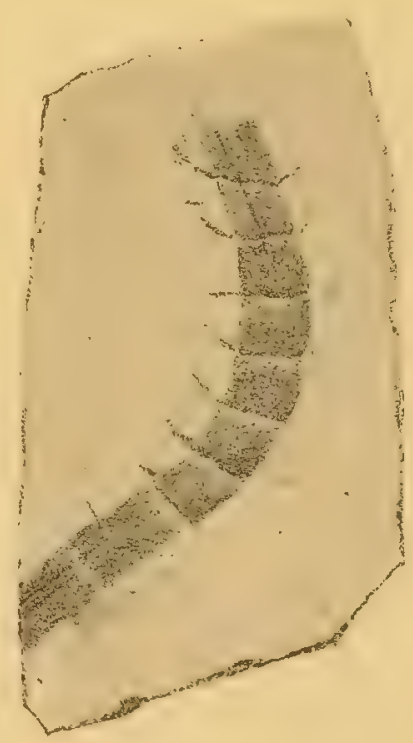

Fig. 147.

certains bois fossiles (Calamariées), où elle est plus souvent représentée par des types de passage entre les ponctuations scalariformes des Ptéridophytes et les vraies aréoles. En outre, Bertrand a cité chez les Psilotacées vivantes des ponctuations aréolées, de nature un peu spéciale, il est vrai, mais bien typiques. 
Daus les types verticillés, comme dans les spiralés, nous avons pu voir souvent les tiges présenter des appendices étranges, jusqu'ici inexplicables (fig. 127, 134, 143, 144, 147).

Les fossiles à disposition verticillée ont reçu les noms les plus variés, ayant été confondus avec le type spirale. Nous nous sommes arrêtés au nom de Psilophyton bohemicum, non pas que nous affirmions être en présence d'une espèce très caractérisée et uettement différente du Psilophyton spinosum, mais pour faciliter la discussion et les descriptions.

Nous detons relever cependant les rapports, tout au moins dans l'apparence générale, entre nos restes verticillés et ceux figurés par Nathorst sous les noms de Sphenophyllum subtenerrimum et de Pseudobornia Visina que Nathorst met parmi les Protocalamariacées, auxquelles nous faisions allusion plus haut.

Il y aurait aussi quelques vagues rapports entre les très mauvais échantillons lécrits par Schubert comme Chondrites Moldave et notre forme à feuilles spiralées ( $C f$. la fig. de Schubert et notre fig. 132 entre autres).

L'allochtonie du terrain ne nous fournit malheureusement que de trop mauvaises formes pour pouvoir élucider tous ces points. Les types spiralés nous ont présenté parfois (fig. 134) un double contour des petites feuilles, contour non attribuable à un précipité minéral. Peut-être seraient-ce dies nervures rappelant un peu celles des Arancariées à larges feuilles. Mais ici, au lieu de plusieurs nervures, nous n'en aurions que deux.

D'autre part, Monsieur R. de Wettstein nous communique qu’il existe dans le Sud du Brésil. dans des tourbières de la région du Camp, une espèce de Lycopodium, qui ressemble plar son apparence extérieure et par sa grandeur à nos Psilophyton. Les branches feuillées de ce Lycopodium sont aplaties et leur structure tout it fait dorsiventrale: elles portent, sur chacun de leurs flancs, une ligne de feuilles, dont les limbes se trouvent sur le même plan que la tige, tandis qu'à la surface de celle-ci, on voit de petites feuilles en forme d'écailles, tantôt disposées en parastiches, tantôt en ligues horizontales rappelant ainsi des verticilles. La partie de la branche qui touche au sol est tout à fait dénuée de feuilles. L'organisation extérieure de cette plante pourrait peut-être nous faire comprendre bien des particularités de nos fossiles.

\section{Incertere sedis.}

1", fig. 14s. Rameau portant des feuilles disposées de façon verticillée comme chez Psilophyton bohemicum. Il y avait également un axe central dans ce rameau principal, lequel portait, en outre, des ramifications curieuses, partant presqu'à angle droit du rameau à feuilles verticillées, pouvant se dichotomiser parfois, et munies de très fines et très nombreuses petites feuilles. Peut-être sommesnous en présence de rameaux jeunes, qui, en s'agrandissant, répéteraient les formes de notre P. bohemicum.

2", fig. 149 et 150. Rameaux portant des feuilles plus ou moins distinctement verticillées. Un fait curieux à relever, c'est que ces feuilles semblaient parfois divisées dichotomiquement (fig. 149). Ceci n'est probablement qu'une apparence et peut provenir de la superposition de deux empreintes foliaires. De plus, ces deux échantillons portaient (visibles surtout dans la figr. 150) de curieuses cicatrices plus ou moins elliptiques et que nous ne savons à quoi rapporter.

30. Dans les fig. 151-155, nous avons représenté des axes, dont nous n'avons pas pu établir les affinités. Ils sont pourvus parfois (fig. 154, 155) de marques qui semblent les Knorria ou des empreintes de feuilles. Plus souvent (fig. 151-153), ces tiges ont l'air de porter des ramifications latérales, qui leur donnent un peu l'apparence de branches de Conifères. 
Coniferites Frilschi, P. et $\mathrm{B}$.

Fig. 156 .

Nous avons eu entre les mains une pièce dont nous possédons aussi la contre-empreinte; elle venait des fouilles que M. Marek a faites près de Srbsko, et consistait en un axe feuillé, les feuilles étant disposées assez régulièrement en alternance d'un côté et de l'autre de l'axe, et étant assez rapprochées les unes des autres.

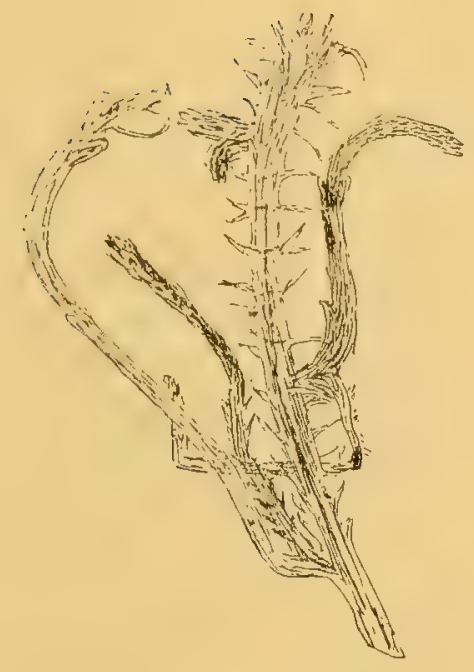

Fig. 148 .

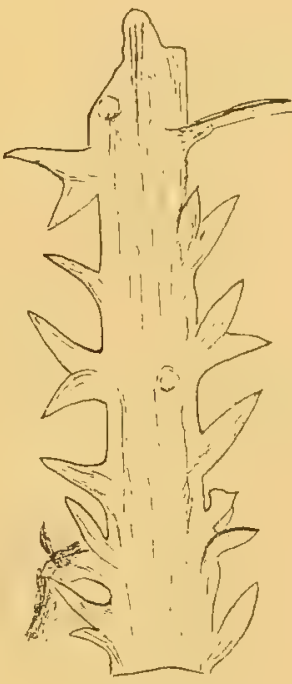

Fig. 149.

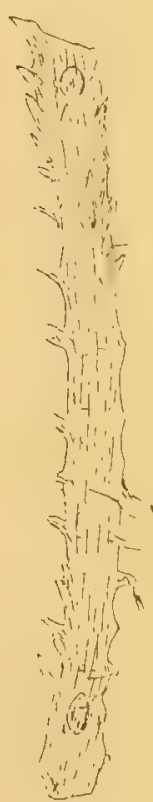

Fig. 150.

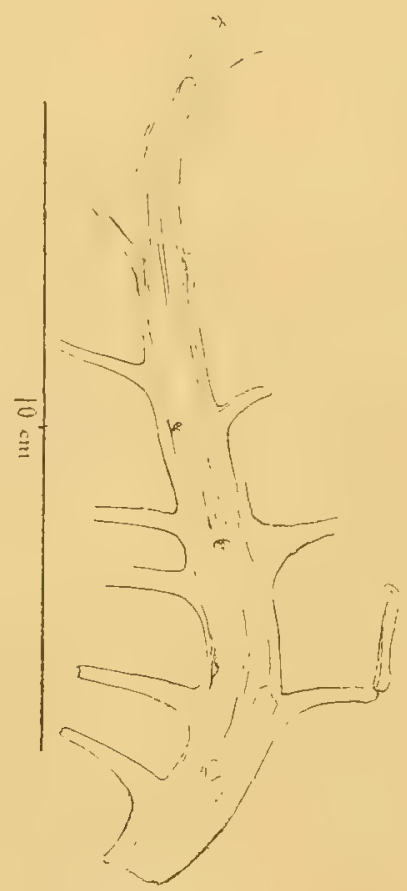

Fig. 151.

Fig. 148-151. Incertae sedis.

148. (Collection Dusl.) Tiameau verticillé. Ramifications latérales à angle droit et portant de très nombreuses et très petites feuilles $\frac{1}{1}$

149. Srbsho. (Geol. Landesmuseum, Berlin.) Feuilles dichotomiques? Curieuses cicatrices arrondies sur le trone $\frac{1}{1}$.

150. " (Geol. Landesmuseum, Berlin.) Feuilles verticillées? dichotomiques? Curieuses cicatrices elliptiques sur le tronc $\frac{1}{2}$.

151. " (K. k. deutsche technische Hochschule, Prag.) Axe ramifié. Knorria?

Nous avons pu constater un caractère curieux de cet échantillon: quelquefois, entre deux feuilles successives, on pouvait remarquer comme un corps arrondi que nous ne savons pas trop comment interpréter. Ces organes, qui contenaient souvent encore de la houille, avaient l'air d'être l'empreinte de sporanges qui, dans ce cas, seraient énormes, ou de semences rappelant celles des cônes de Conifères. Mais il est plus probable encore que ce soient tout simplement des feuilles qui se présenteraient de face, tandis que les autres, dont nous parlions ci-dessus, étroites et serrées les unes contre les autres, seraient simplement des feuilles, assez larges peut-être, mais considérées sur leurs tranches. Des branches assez semblables ont été décrites comme Conifères, entre autres par M. de Saporta, qui, dans le Calcaire jurassique de la "Porte de France“ à Grenoble, a trouvé un fossile qu'il désigne sous le nom de Pachyphyllum crassifotium, Schenk, et qui présente dans sa disposition générale une 
certaine analogie avec le type qui nous occupe. L'auteur dit (1.655): "Ce rameau, sans doute figé, „est jourvu de feuilles rigides, trigones, étalées et recourbées en faux, et qui s'élèvent sur une base „légèrement conique et (lécurrente, qui constitue à chacune d'elles un coussinet saillant, étroitement

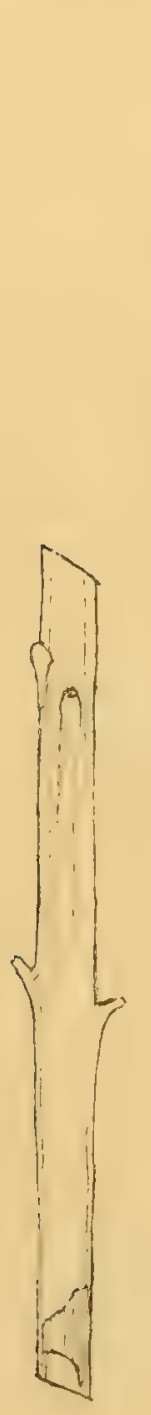

Fig. 152,

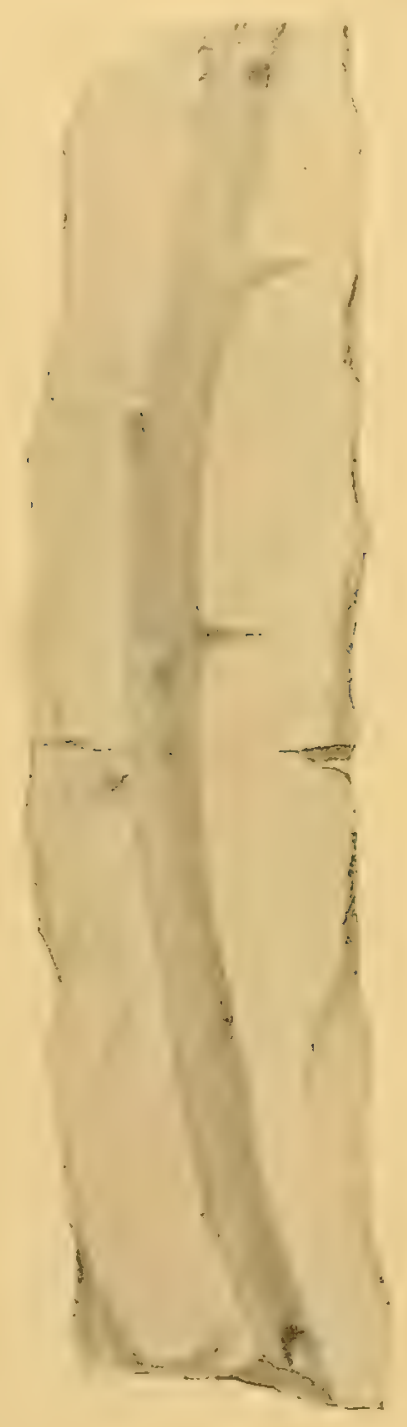

Fig. 153.

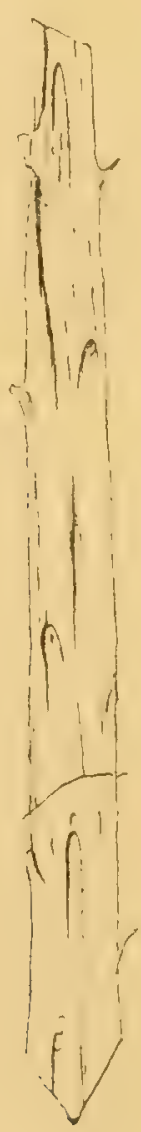

Fig. 154.

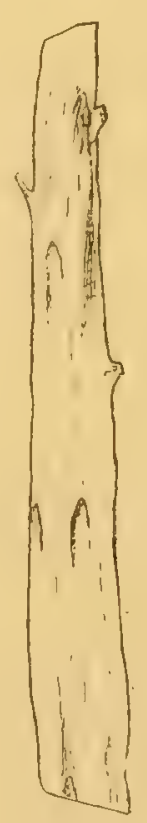

Fig. 155.

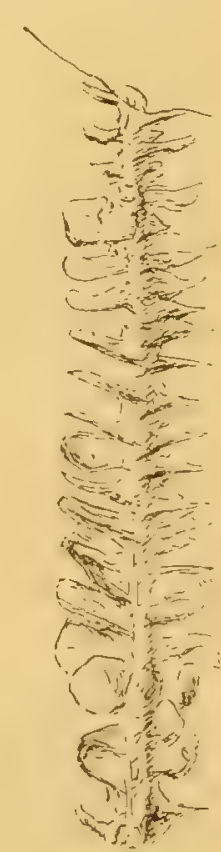

Fig. 156.

Fig. 152-155. Incerta sedis.

152. Hostim. (Univ. Wien) Axe ramifié -1.

153. (K. k. geol. Reichsanstalt.) Axe ramifié ?

15.1-155. Srbsko. (Univ. Wien.) Axes avec feuilles (?) it

Fig. 156. Coniferites Fritschi, P. et B.

156. Srbsko. (Geol. Laudesmuseum, Berlin.) Axe feuillé $\frac{1}{1}$.

,serré contre le coussinet de la feuille limitrophe. Par un effet de la compression, les feuilles laté"rales, vues de profil, ont été seules conservées." Ce dernier caractère pourrait être en rapport avec ce que nous avons signalé pour notre type. Nous n’avons pas pu reconnaître tous ces détails sur 
notre échantillon, qui est encore plus mal conservé que celui figuré par. Saporfa. Cependant nous tenions à relever l'analogie curieuse de certains points, et, jusquà plus ample informé, nous placerons ce fossile parmi les Conifères, dans le voisinage de Pachyphyllum crassifolium; nous l'avons dédié it Monsieur le Professeur Anton Fritsch, de Prague, sous le nom de Coniferites Fritschi.

Ln résumé, nous sommes en présence des résultats les plus importants suivants:

1. La flore du Dévonien moyen de la Bohême (Srbsko, Hostím etc.) est nettement caractérisée comme flore allochtone.

$2^{0}$. Cette flore allochtone est certainement terrestre. La présence d'axes centraux très distincts, de Knorria et d'Aspidiaria, de charbon à la surface des empreintes, d'éléments vasculaires, etc., prouve que ces plantes ne sont pas des Algues. Nous n'avons trouvé aucun reste végétal qui nous permît de penser que nous avions affaire à une Algue, quand même nous étions en présence de schistes marins.

3". Il est plus ou moins certain que ces plantes appartiennent aux groupes végétaux suivants: Fougères, Protocalamariacées, Psilotacées (ou type mixte), Bothrodendracées, Lycopodiacées ou Sélagimellacées, Ginkigoacées, Conifères.

4". Pour faciliter la discussion et mettre un peu de clarté dans la bibliographie confuse et dans la synonymie embrouillée de ces fossiles, mais sans vouloir attribuer à nos espèces aucun caractère définitif, nous avons donné à nos échantillons les noms ci-après, et nous les caractérisons par les courtes diagnoses suivantes:

1. Spiropteris hostimensis: Frondes jeunes de Fougères avec extrémités encore enronlées en crosses.

2. Rhodea (?) hostimensis: Restes très semblables à Rhodea (Fougère) et rappelant notamment R. Condrusorum du Dévonien.

3. Hostimella hostimensis a) typica: Axes ramifiés, pennés par "Uebergipfelung"; formations à apparence de bourgeons à la base des articles des rameaux.

$\beta$ ) rhodereformis: comme $\alpha$, mais plus grande, sans ces "bourgeons" ou du moins, ceux-ci ì peine distincts ou rarement présents. Plus abondante ramification.

4. Cf. Asterocalamites scrobiculatus: Axe dichotome (?). Le reste, comme on l'a décrit pour les moulages internes d'Asterocalamites.

5. Pseudosporochnus Krejčí: Plantes avec des troncs pouvant atteindre jusqu'à $2^{m}$ environ de hauteur et jusqu'à $2^{d m}$ de largeur; ils sont élargis à la base, et en haut se dichotomisent en éventail ; enfin ils se terminent par de très fines ramifications pennées et dichotomes, dont les extrémités ultimes sont dichotomes et renfeées en massues (sporanges?). La surface des trones est knorrioïde, du type Knorria acicularis.

6. Protolepidodendron larlsteini: Etroits rameaux avec de petites feuilles très serrées, ovaleslancéolées; la surface des rameaux est couverte de coussinets lépidodendroïdes (bergérioïdes).

7. Protolepidodendron Scharyamum: Comme 6, mais les feuilles sont étroites et terminées au sommet par une petite dichotomie.

8. Ulodendron(?) hostimense: Axe épais et ramifié en dichotomie; marques ulodendroïdes.

9. Lycopodites hostimensis: Restes analogues à Lycopodium, avec de gros sporanges.

10. Barrandeina Dusliana: Grands troncs dichotomes. Etats de conservation knorrioïdes, bergérioïdes ou aspidiarioïdes. Feuilles du type Ginkgo. 
11. Psilophyton spinosum: Epais rameaux avec feuilles écailleuses, disposées en spirales et souvent assez espacées les unes des autres. Il se trouve presque toujours, au centre du rameau un axe longitudinal (faisceau ou moelle?).

12. Psilophyton bohemicum: Comme 11, mais les appendices (feuilles) sont disposés en verticilles.

13. Coniferites Fritschi: Rameau analogue à une branche de Conifere, et dont l'habitus rappelle le Pachyphyllum crassifolium, Schenk, décrit par de Saporła.

En outre, quelques nincerte sedis "i qui devraient peut-être être rangés dans d'autres catégories.

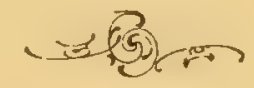




\section{Littérature.}

J. Barrande. 1846. Note préliminaire sur le système silurien et les Trilobites. Leipzig.

- 1852. Système silurien du centre de la Bohême. Vol. I, p. 71 et 72.

- 1854. Beobachtungen über die Kruster, Flossenfüsser und Kopffüsser des böhmischen Silurgebirges. Neues Jahrbuch, p. 1, Stuttgart.

- 1865. Défense des Colonies III. Etude générale sur les étages G-H. Prague et Paris.

- 1865. Défense des Colonies III. Referat im Neuen Jahrbuch, p. 231.

- 1866. Beleuchtung einiger Ansichten über die Colonjen. Neues Jahrbuch, p. 293.

- 1870. Défense des Colonies IV. Prague et Paris.

E. Bayer: 1900. Referat ciner Arbeit von Mhařil: Príspěvek fo floře českého devonu. Just's Jahresbericht, XXVIII, 2. Abt. p. 211.

- 1903. In litteris.

C. E. Bertrand. 1882. Recherches sur 7es Thésiptéridées. Archives botaniques du Nord de la France. $2^{e}$ année, $\mathrm{N}^{\prime \prime} 17$, p. 252.

L. Criẻ. 1883. Essai sur la flore primordiale. Les origines de la vie. Paris.

J. W. Dawson. 1862. On the Flora of the Devonian Period in North Eastern America. Quart. Journ. Geol. Soc. XVIII, 1) 296.

- 1863. Further Observations on the Devonian Plants of MLaine, Gaspé and New-Iorle. Quart. Journ. Geol. Soc. XIX, p. 1862.

- 1870. On the prinitive vegetation of the Earth. Quart. Journ. Geol. Soc. Montréal, p. 165.

- 1871. The fossits plants of the Devonian and upper Silurian formations of Canada. Geological Survey of Canada. Montréal.

- 1873. Report on the fossil Plants of the Lower Carboniferous and IIillstone Grit formations of Canada. Geol. Survey of Canada. Montréal.

- 1880. Notes on New Erian (Devonian) Plants. Quart. Journ. Geol. Soc. XXXVII, p. 299.

- 1888. The Geological history of Plants. London.

C. von Ettingshausen. 1881. In litteris.

F. Frech. 1886. Ueber die nähere Altersbestimmung der Etage F-G-H Barrande's. Zeitschr. d. d. Geol. Ges. XXXVIII, 1. 917.

H. R. Goeppert. 1860. Weber die Fossile Flora der silurischen, der devonischen und unteren Kohlenformation, oder des sog. Uebergangsgebirges. Nova acta, Vol. XXVII. Jena.

0. Heer. 1867. Flora fossilis arctica. Vol. I. Zürich.

- 1871. Fossile Flora der Bären-Insel. Svensk. Vet. Akad. Handlingar. Vol. 9. Nr. 5. Stockholm.

J. J. Jahn. 1903. Ueber diej Etage $H$ im mittelböhmischen Devon. Verhdl. d. k. k. geol. Reichsanstalt in Wien, Nr. 4 .

F. Katzer. 1902. Geologie von Böhmen. Prag.

E. Kayser. 1880. Zur hercynischen Frage. Jahrb. d. k. k. geol. R. A., p. 557. 
E. Kayser. 1884. Ueber die Grenze zwischen Silur und Devon (Hercyn) in Böhmen, Thüringen, etc. Neues Jahrbuch. Vol. II, 1. 81.

J. Krejči. 1879. Notiz ïber die Reste von Landplanzcn in der böhmischen Silurformation. Sitzungsberichte d. k. böhm. Ges. d. Wissensch. Prag. 4 avril, p. 201.

- 18S1. Ueber ein nenes Vorkommen von Landpflanzen und Fucoiden in der böhmischen Silurformation. Sitzungsber. d. k. böhı. Gesell. d. Wissensch. Prag. 11 Février, 1. 68.

Mac' Coy. 1876. Prodromus of the Paleontology of Victoria. Decade IV. Melburn et London.

V. Mařik. 1900. Př́splèvek k floře čestího devonu. (Contribution à la flore du Dévonien de Bohême.) Rozpravy české akademie císaře Františka Josefa pro vědy, slovesnost a umèní. IX année, $\mathrm{II}^{e}$ classe, Nr. 18. Prag.

A. G. Natharst. 1894. Zu* fossilen Flora der Polarländer, I. Theil, I. Lieferung: Zur paläozoischen Flora der arlitischen Zone. Stockholm.

- 1902. Zur fossilen Flora der Polarländer. I. Theil. III. Lieferung: Zur oberdevonischen Flora der Büren-Insel. Stockholm.

J. Palacký. 1895. Ueber die Concordanz der New-Iorker Erian-Flora mit der böhmischen sog. hercymischen. Sitzungsber. d. k. böhn. Gesellsch. d. Wissensch. 8 férrier.

D. P. Penhallow. 1893. Notes on Erian (Deromian) Plants from Nev-Yorli and Pennsylvania. Smithson institution. Proceedings of the U.-S. Nat. Museum: Vol. IVI, p. 105.

H. Potonie. 1895. Die Beziehung zwischen dem echt-gabeligen und dem fiecterigen Werlel-Aufbau der Farne. Berichte der Dentschen botanischen Gesellschaft. Berlin.

- 1899. Lehrbuch der Pflanzenpalcontologie. Berlin.

- 1901. Die Silur- und CuTm-Flora des Harzes und des Magdeburgischen. Abhandl. d. k. Preuss. Geol. Landesanstalt. N. F. Heft 36. Berlin.

- 1902. Varia dans Engler und Prantl's natürlichen Pflanzenfamilien: Pteridophyten. I. 4, Leipzig.

- 1903. Lin Blick in die Geschichte der botanischen Morphologie und die Pericaulom-Theorie. Evweiterter Abdruck aus del Naturwiss. Wochenschr. N. F. II. Bd. Der ganzen Reihe, XVIII. Bu. Jena.

- 1903. Ueber tie Flora des Etaye $1 \%$, in J.-J. Jahn: Ueber die Etage H im mittelböhmischen Devon. Verhandl. d. k. k. geol. Reichsanstalt in Wien. Nr. 4.

A. Rothpletz. 1896. Ueber die Flysch-Fucoiden und einige andere fossile Algen, souvie übè liasische Diatomeen fiihrende Hornschroämme. Zeitschr. d. deutschen geol. Gesellsch. Berlin, p. 854.

G. de Saporta. 1854. Paléontologic française, III. Paris.

Schenck. 1890. Palcophytologie. II. Teil rles Zittel's Handbuchs der Palrontologie. München et Leipzig.

R. J. Schubert. 1899. Chondrites Molduve Schub, cin Algenrest aus dem böhmischen Obersilur. Nenes Jahrbuch. Vol. I, p. 129.

H. zu Solms-Laubach. 1895. Ueber devonische Pflanzenreste aus den Lemeschiefern rer Gegend von Gräfrath am. Niederrhein. Jahrb. d. k. jureuss. geol. Landesanstalt für 1894. $2^{e}$ lartie, 1\%.67. Berlin.

D. Stur. 1852. Die Silur-Flora ner Etage $H-h 1$ in Böhmon. Sitzungsber. der Matl.-naturw. Classe d. k. böhm. Ges. d. Wiss., LXXXIV, 1. Abt., 1) 330.

R. von Weltstein. 1903. In litteris.

R. Zeiller. 1900. Eléments de Palíbotanique. Paris. 


\section{N D E X.}

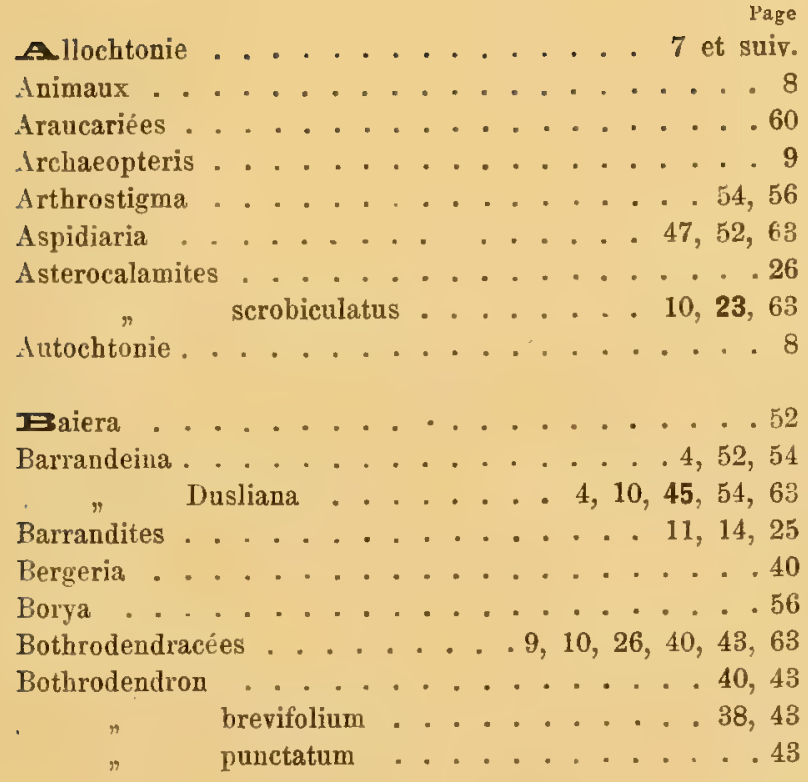

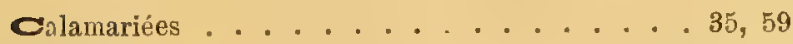

Calamitées . . . . . . . . . 26

Calamites ................. 52

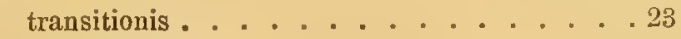

Callithamnion Reussianum ......... 2

Calymmotheca . . . . . . . . . . 36

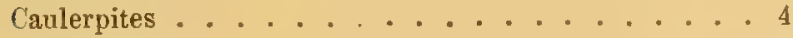

Characées .............. 4, 51, 56

Chauvinia Scharyana....... 3, 4, 38, 40

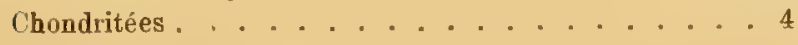

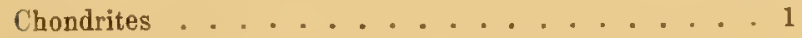
fruticulosus ............... 2

$\beta$ subarticulatus . . . . 2

Moldavae ......... 5, 50

verticillatus ....... . . 3, 25

Codonophyton epiphyticum ........... 35

Confervites ..................... 3

Conifères . . . . . . . 9, 10, 58, 60,61,63

'oniferites Fritschi ....... 10,61,64

Cordaites australis . . . . . . . . . . 35

borassifolius .......... 3,51

Cryptogames vasculaires ......... 4, 5

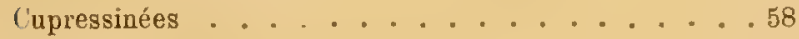

Cycadofilices ......... 35,36

Cyclopteris . . . . . . . . . . . 52

incerta............. 14
Page

Cyclostigma brevifolium . . . . . . 38, 4 ;

Cystoseira ................... 3

Delesserites . . . . . . . . . 3, 51

Dicranophyllum . . . . . . . . . 41,43 australicum . . . . . . 38, 41

Dictyophycées .............. . . . 4

Dictyotites . . . . . . . . . . . . . . . . . . . .

Dimepteris . . . . . . . . . . . . 36

Drepanophyllum spinaeforme ........ 3

Eophyton . . . . . . . . . 4

Equisetales ............ 10, 24,41

Equisetites siluricus .......... . . . .

Gopperti ...................

Flabellaria borassifolia . . . . . . . . . . 51

Floridées . . . . . . . 2, 3, 5, 11, 13

Fougères . . . . . . . . . . . . . . . . . . . 10,63

Fucacées . . . . . . . . . . 56

Fucoïdées . . . . . . . . . . . . . 4

Fucoïdes . . . . . . . . . 1, 2,3

hostinensis . . . . . 2, 3, 11, 13, 14

Fucus .................. . . 54

,$\quad$ Nováki ......... 3, 4, 54,56

Gigartina ............... 13

" pistillata............. . 13

Gigartinées . . . . . . . . . . . 3,11

Ginkgo . . . . . . . . . . . . 63

Ginkgoacées . . . . . 9, 10,48,50,52,63

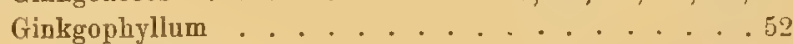

Gymnospermes ........... 4, 10, 85, 52

Iräcksel .................. 8

Haliserites . . . . . . . . . 3, 54, 56 spinosus ......... . . . 3,54

zonarioides ........... 3, 13,11

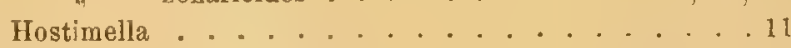
hostimensis . . . . . 10, 11, 14,63 typica . . . . 10, 14, 6: rhodeaeformis . . . 10, 20,68

Hostinella ........... 11, . . . . . . . hostinensis . . 3, $4,5,8,11,13,11,25$ Hyménophyllacées . . . . . . . . . 35

I-norria . . . 3, 25, 32, $35,36,38,43,46,52,60,63$ acicularis .........26,63 
I.aminariées ............. 3, 5 . .

Lépidodendracées ....... . 26,43, 45, 47, 54

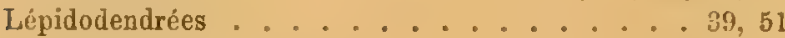

Lepidodendron ............. . 36, 43

Gaspianum ............... 43

Veltheimi ......... 3, . 37

Veltheimianum ...... 11, 38, 40

Lépidophytes ................. 36, 40

Lessonia ........................ 54

, boheuica.......... 3, 4, 54, 56

.. fuscescens ........... 56

Lycopodiacées ........... 10, 45, 56, 63

Lycopodiales .................. 9, 10,36,58

Lycopodites...................... 45, 63 hostimensis ........... 10, 45

Lycopodium . . . . . . . . . 45,60,63

Lyginopteridées ........................ 35

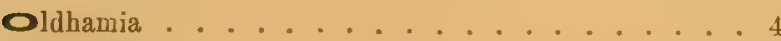

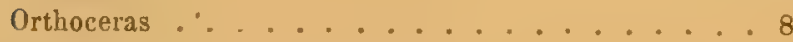

Pachyphyllum crassifolium ......... 61, 61

Palaeophycées................. 4

Pericaulom .................... 52

Phaeophycées . . . . . . . . . . . 57

Irotocalamariacées.......... 9, 41, 58, 63

Protolepidodendron .......... 38, 45,54

Duslianum .... $3,4,45,51,54$

karlsteini ..... 10, 38, 39, 63

Scharyanum ... $3,10,38,40,63$

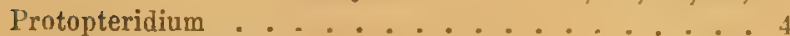

n hostinense :............ 11, 12

Pseudobornia ursina . . . . . . . . . . 60

Pseudosporochnus ............... 23

" Krejčíi........ 10, 25, 63

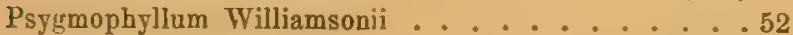

Psilophyton ............ $54,56,60$ bohemicum ..... 10,54,58,60,63 grande ............. 54 princeps ............. . . 54

Psilophyton spinosum ........ 10, 54, 58, 64 Psilotacées ....... 9, 10, 35, 36, 54, 56, 59, 63 Psilotum .................. 36 $\rightarrow$ triquetrum ............................ Pteridium . . . . . . . . . . . . . . . Ptéridophytes . . . . . . . . . 5, 8, 59

I hachiopteris tenuistriata ........... 23

Rhacophyton condrusorum ............. . 14 Rhodea............ 9, 13, 20,22,63 condrusorum .............. 14, 63 (?) hostimensis .......... 10, I3, 63

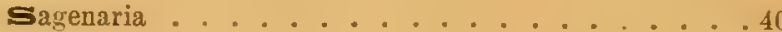

Sargassites .............. 54, 56

Schizaea . . . . . . . . . . . . . 11

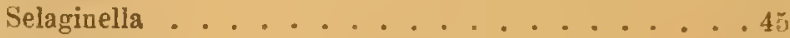

Sélaginellacées .............. 45, 63

Siphonées ............. 3, . . 40

Sphaerococcites Scharyanus ............ 2

Sphénophyllacées ................ 58

Sphenophyllum subtenerrimum .........60 60

Sphenopteridium Keilhaui .......... 14, 22

Sphenopteris condrusorum ............. 14

Velenovskýi . . . . . . . . 5

Spiropteris ............. 9, 13, 22 hostimensis......... 10, 11, 63

Sporochnoïdées . . . . . . . . . . . s

Sporochnus Krejčii ........ 3, 4, 13, 25, 35

Spongiophycées ................ 4

Thallophytes ............... 8

Truésipteridées . . . . . . . . . . . . 35

Tmesipteris . . . . . . . . . . . . 36

Trilobites................ 8

Ulodendron (?) hostimense ........ 10, 43, 63 punctatum ................ 45

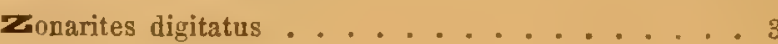





(5) $\left.)^{2}-6\right)^{2}=\sqrt{3}$ 